\title{
Stability of Slender Bodies under Compression and Validity of the von Kármán Theory
}

\author{
Myriam Lecumberry \& Stefan Müller
}

\author{
Communicated by THE EDITORS
}

\begin{abstract}
Since their formulation almost 100 years ago, the von Kármán (vK) plate equations have been frequently used both by engineers and by analysts to study thin elastic bodies, in particular their stability behaviour under applied loads. At the same time the derivation of these equations met some harsh criticism and their precise mathematical status has been unclear until very recently. Following up on a recent variational derivation of the vK theory by Friesecke, James and Müller from three-dimensional nonlinear elasticity we study the predictions and the validity of the vK equation in the presence of in-plane compressive forces. The first main result is a stability alternative: either the load leads to instability already in the nonlinear bending theory of plates (Kirchhoff-Love theory), or it leads to an instability in a geometrically linear KL theory ('linearized instability'), or vK theory is valid. The second main result states that under suitable conditions the critical loads for nonlinear stability and linearized instability coincide. The third main result asserts this critical load also agrees with the load beyond which the infimum of the vK functional is $-\infty$. The main ingredients are a sharp rigidity estimate for maps with low elastic energy and a study of the properties of isometric immersions from a set in $\mathbb{R}^{2}$ to $\mathbb{R}^{3}$ and their geometrically linear counterparts.
\end{abstract}

\section{Contents}

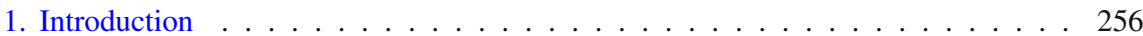

2. Main results . . . . . . . . . . . . . . . . . . . . 258

2.1. The energy . . . . . . . . . . . . . . . . . . . . . . 258

2.2. The scaling . . . . . . . . . . . . . . . . . . . . . . 259

2.3. The boundary conditions . . . . . . . . . . . . . . . . . . . 260

2.4. Normal forces . . . . . . . . . . . . . . . . . . . . . . . . 261

2.5. Normal and in-plane forces-the stability alternative . . . . . . . . . . 261

2.6. Nonlinear stability versus linearized stability . . . . . . . . . . . . . . . . 263

2.7. Main ingredients of the proof . . . . . . . . . . . . . . . . . 264 
2.8. Related work . . . . . . . . . . . . . . . . . . . . . . . . 264

2.9. Outline . . . . . . . . . . . . . . . . . . . . . 265

3. Geometric rigidity . . . . . . . . . . . . . . . . 265

4. Scaling of in-plane and out-of-plane components and limiting strain . . . . . . . . 267

4.1. Results without boundary conditions . . . . . . . . . . . . . . . . 267

4.2. Clamped boundary conditions . . . . . . . . . . . . . . . 268

4.3. Clamped boundary conditions for the out-of-plane component . . . . . . . 271

4.4. Identification of the limiting strain . . . . . . . . . . . . . . . . . . . 273

5. Derivation and range of validity of the vK theory . . . . . . . . . . . . . 274

5.1. Normal forces . . . . . . . . . . . . . . . . . . . . . . 274

5.2. Normal and in-plane forces; stability alternative . . . . . . . . . . . . . . . 278

6. Nonlinear stability versus linearized stability . . . . . . . . . . . . . . . . . 281

7. An example: a rectangular plate under compression . . . . . . . . . . . 285

7.1. Euler-Bernoulli theory . . . . . . . . . . . . . . . . . . . . . . 286

7.2. Geometrically linear stability . . . . . . . . . . . . . . . . . 287

7.3. Nonlinear stability . . . . . . . . . . . . . . . . . . . . . . . . . 288

7.4. First summary . . . . . . . . . . . . . . . . . . . . . . . . . . . . . . . 288

7.5. Behaviour below $p_{\mathrm{cr}}$, vK buckling . . . . . . . . . . . . . . . . 288

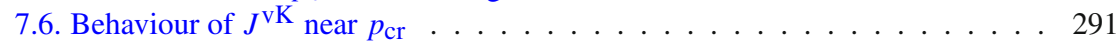

7.7. Summary . . . . . . . . . . . . . . . . . . . . . . . 297

8. The relation between attainment for $J^{\mathrm{vK}}$ and linearized stability . . . . . . . . . 298

8.1. Homogeneous out-of-plane boundary conditions . . . . . . . . . . . . . . . . 299

8.2. Non-homogeneous out-of-plane boundary conditions . . . . . . . . . . . . 301

9. Some facts about isometries and infinitesimal isometries . . . . . . . . . . . 303

9.1. Construction of isometries . . . . . . . . . . . . . . . . 303

9.2. Simple regularity estimates . . . . . . . . . . . . . . . . . . 306

9.3. Refined regularity estimates . . . . . . . . . . . . . . . . 306

\section{Introduction}

Since the work of BoOBNOFF ${ }^{1}$ in 1902 [2,3], FöPPL in 1907 [9, pp. 132-144] and VON KÁRMÁN in 1910 [20, pp. 348-352] the von Kármán plate equations have been widely used to study the behaviour of thin elastic bodies, in particular their stability behaviour. Despite their popularity by engineers and nonlinear analysts alike, the vK equations have also faced very harsh criticism. TRUESDELL [38, pp. 601-602] has strongly criticized their usual derivation (the main points are summarized in the introduction of [4]) and VILlagGio [39, p. 4] cites the vK equations as an example of a 'bad theory' in his textbook on structural mechanics. In his threevolume essay on nonlinear elasticity, plate and shell theory, CiARLET writes [5, p. 367]: 'The two-dimensional von Kármán equations for nonlinearly elastic plates, originally proposed by T. von Kármán in 1910, play an almost mythical role in applied mathematics'.

1 I.G. Boobnoff (Иван Григорьевич Бубнов, also transcribed as I.G. Bubnov or I.G. Bubnow) realized the importance of considering both bending and stretching terms for the design of ships' plating and constructed approximate solutions by replacing the in-plane stresses in the equation later written down by von Kármán by an averaged hydrostatic tension term. We thank F. Duderstadt for bringing Boobnoff's work to our attention, see [6, pp. 103104] for further information. 
The situation has become clearer recently through the use of variational methods. In particular it has been shown in [13] that vK functional (whose Euler-Lagrange equations are the $\mathrm{vK}$ equations) arises as a $\Gamma$-limit of nonlinear three-dimensional elasticity if the energy per unit volume scales like thickness $h$ to the fourth power. In that paper boundary conditions are not discussed in detail to keep the exposition simple, and only normal loads are considered. To understand to what extent the vK equations can capture instability phenomena such as buckling it is, however, crucial to include boundary conditions and in-plane loads. This is done the in current paper.

Our first main result is the following stability alternative (see Theorem 4 below for a precise statement):

- Either the load is strong enough to cause a nontrivial deformation (of order one) in the nonlinear bending theory of plates, first proposed by KIRCHHOFF [22] ('nonlinear instability'),

- or the load leads to an instability in a geometrically linear version of Kirchhoff's theory ('linearized instability'),

- or vK theory applies (and the deformations are of order $h^{2}$ in plane and of order $h$ out-of-plane).

The different regimes can be related to different scalings of the energy $e^{h}$ per unit volume as a function of the thickness: nonlinear instability corresponds to $e^{h} \sim h^{2}$, linearized instability to $h^{4} \ll e^{h} \ll h^{2}$, and the vK regime is characterized by $e^{h} \sim h^{4}$.

Our second result shows that failure of linear and nonlinear stability occurs at the same critical load, at least if the region where the body is clamped is connected (see Theorems 6 and 7 below).

Our third result asserts that linearized stability is closely related to the existence of minimizers of the vK energy. For homogeneous boundary conditions (that is, zero out-of-plane displacements are permissible) we show that the infimum of the vK energy is $-\infty$ if and only if the load exceeds the critical load for linearized stability (see Theorem 27 below).

To summarize we show (under the conditions stated) that the vK functional captures well the asymptotic behaviour of three-dimensional minimizers until a critical load is reached. This critical load can be characterized by three equivalent conditions

$$
\text { linearized instability } \Leftrightarrow \text { nonlinear instability } \Leftrightarrow \inf J^{\mathrm{vK}}=-\infty
$$

Typically there is a lower threshold for which the trivial solution of the vK equations is no longer minimizing ('vK buckling'). In certain situations (for example, for zero Poisson's ratio) it may happen that the trivial $\mathrm{vK}$ solutions is minimizing up to the complete breakdown of vK theory in the sense of (1). In this case vK theory nonetheless detects the onset of a nonlinear instability through the fact that the infimum of the vK functional is $-\infty$ for loads above the critical one.

To illustrate these results we consider the example of a rectangular plate clamped at one end and uniformly loaded at the opposite end. Other interesting examples arise when both linearized and nonlinear stability always hold (this is, for example, the case for a plate which is clamped throughout, but admits tangential motion of 
the boundary). In this case the vK equations always apply (for in-plane forces of order $h^{2}$ ). If one works with dead loads, some care, however, is needed to formulate the three-dimensional problem and the Kirchhoff problem correctly, so as to avoid 'unphysical' solutions, such as a 180 degree rotation of a circular plate in uniform compression. These issues will be discussed in more detail elsewhere.

Very roughly speaking, boundary conditions for which nonlinear and linearized stability always hold lead to small deflections even after the critical first buckling load, while plates subject to boundary conditions for which these stability conditions fail show a much softer 'beam-like' buckling response with large deflections after the failure of the stability conditions. We are grateful to E. Ramm for pointing out to us that taking into account a 'beam-like' behaviour (as exhibited by our example in Section 7) is important for engineering design, for example, for certain bridge constructions.

\section{Main results}

We now begin with a more formal description of our results.

\subsection{The energy}

We start from the elastic energy

$$
\mathcal{E}^{h}(w)=\int_{\Omega_{h}} W(\nabla w(z)) \mathrm{d} z
$$

of a deformation

$$
w: \Omega_{h}=S \times\left(-\frac{h}{2}, \frac{h}{2}\right) \rightarrow \mathbb{R}^{3} .
$$

It is convenient to work in a fixed domain $\Omega=S \times\left(-\frac{1}{2}, \frac{1}{2}\right)$, change variables $x=\left(z_{1}, z_{2}, \frac{z_{3}}{h}\right)$ and rescale deformations according to $y(x)=w(z(x))$ so that $y$ : $\Omega \rightarrow \mathbb{R}^{3}$. We abbreviate $x^{\prime}=\left(x_{1}, x_{2}\right)$ and use the notation $\nabla^{\prime} y=y_{, 1} \otimes e_{1}+y_{, 2} \otimes e_{2}$ for the in-plane gradient so that

$$
\nabla w=\left(\nabla^{\prime} y, \frac{1}{h} y_{, 3}\right)=: \nabla_{h} y
$$

and

$$
\frac{1}{h} \mathcal{E}(w)=I^{h}(y):=\int_{\Omega} W\left(\nabla_{h} y\right) \mathrm{d} x .
$$

We assume that the stored energy $W$ is frame indifferent and has a nondegenerate minimum at the group $S O(3)$ of rotations. More precisely, we suppose 
that $W$ is Borel measurable with values in $[0, \infty]$ and satisfies

$$
\begin{gathered}
W(Q F)=W(F) \quad \forall Q \in S O(3), \\
W=0 \quad \text { on } \quad S O(3), \\
W(F) \geqq c \operatorname{dist}^{2}(F, S O(3)), \quad c>0, \\
W \text { is } C^{2} \text { in a neighbourhood of } S O(3) .
\end{gathered}
$$

Since the relevant deformation gradients will be close to $S O$ (3) (at least in an $L^{2}$ sense), we also consider the quadratic form

$$
Q_{3}(F)=\frac{\partial^{2} W}{\partial F^{2}}(I d)(F, F),
$$

which is twice the linearized energy, and $Q_{2}: \mathbb{R}^{2 \times 2} \rightarrow \mathbb{R}$,

$$
Q_{2}(G)=\min _{a \in \mathbb{R}^{3}} Q_{3}\left(G+a \otimes e_{3}+e_{3} \otimes a\right)
$$

obtained by minimizing over stretches in the $x_{3}$ directions. In view of (5) and (6) both forms are positive semidefinite and hence convex. For the special case of isotropic elasticity we have

$$
\begin{aligned}
& Q_{3}(F)=2 \mu\left|\frac{F+F^{T}}{2}\right|^{2}+\lambda(\operatorname{tr} F)^{2}, \\
& Q_{2}(G)=2 \mu\left|\frac{G+G^{T}}{2}\right|^{2}+\frac{2 \mu \lambda}{2 \mu+\lambda}(\operatorname{tr} G)^{2} .
\end{aligned}
$$

We study the behaviour of (almost) minimizers of the functionals

$$
J^{h}(y)=\int_{\Omega} W\left(\nabla_{h} y\right)-f^{(h)}\left(x^{\prime}\right) y_{3} \mathrm{~d} x-\int_{\partial S \times(-1 / 2,1 / 2)} g^{(h)}\left(x^{\prime}\right) \cdot\left(y^{\prime}-x^{\prime}\right) \mathrm{d} x^{\prime} .
$$

This corresponds to normal body forces $f^{(h)}$ and tangential boundary forces $g^{(h)}=$ $\left(g_{1}^{(h)}, g_{2}^{(h)}\right)$. More general forces can also be considered. We have focused on the above choice for its simplicity and since it already captures the typical instability phenomena.

\subsection{The scaling}

If $e^{h}:=I^{h}\left(y^{(h)}\right)=\int W\left(\nabla_{h} y^{(h)}\right)$ is bounded by $C h^{2}$ then a subsequence of $y^{(h)}$ converges strongly in the Sobolev space $W^{1,2}$ to $\bar{y}$ (see [12, Theorem 4.1]). The limit map $\bar{y}$ is independent of $x_{3}$ (and may be viewed as the deformation of the mid-plane of the plate) and, as a map from $S$ to $\mathbb{R}^{3}$, is an isometric immersion. If $e^{h} \ll h^{2}$ then the limit map is even a rigid motion. In view of the boundary conditions which we will introduce below, we obtain $\bar{y}\left(x^{\prime}, x_{3}\right)=\left(x^{\prime}, 0\right)$. The vK theory corresponds to the scaling $e^{h} \sim h^{4}$ and we introduce the scaled in-plane and 
out-of-plane displacements $u^{(h)}, v^{(h)}$ which measure the deviation of $y^{(h)}$ from the trivial map $x \mapsto\left(x^{\prime}, 0\right)$.

$$
u^{(h)}\left(x^{\prime}\right):=\frac{1}{h^{2}} \int_{I}\left(\begin{array}{l}
y_{1}^{(h)} \\
y_{2}^{(h)}
\end{array}\right)\left(x^{\prime}, x_{3}\right)-\left(\begin{array}{l}
x_{1} \\
x_{2}
\end{array}\right) \mathrm{d} x_{3}, \quad v^{(h)}\left(x^{\prime}\right):=\frac{1}{h} \int_{I} y_{3}^{(h)} \mathrm{d} x_{3},
$$

where

$$
I=(-1 / 2,1 / 2)
$$

We shall see later in Lemma 13 that under the assumption $e^{h} \leqq C h^{4}$ and suitable boundary conditions we have $u^{(h)} \rightarrow u$ and $v^{(h)} \rightarrow v$ in $W^{1,2}$, with $v \in W^{2,2}$.

For general $u \in W^{1,2}\left(S, \mathbb{R}^{2}\right)$ and $v \in W^{2,2}(S)$ we introduce the von Kármán functional

$$
I^{\mathrm{vK}}(u, v):=\int_{S} \frac{1}{2} Q_{2}\left(\operatorname{sym} \nabla^{\prime} u+\frac{1}{2} \nabla^{\prime} v \otimes \nabla^{\prime} v\right)+\frac{1}{24} Q_{2}\left(\left(\nabla^{\prime}\right)^{2} v\right) \mathrm{d} x^{\prime},
$$

where we denote the symmetric part of a matrix $F$ by

$$
\operatorname{sym} F=\frac{F+F^{T}}{2} \text {. }
$$

To include applied forces we also consider the functional

$$
J^{\mathrm{vK}}(u, v)=I^{\mathrm{vK}}(u, v)-\int_{S} f v \mathrm{~d} x^{\prime}-\int_{\partial S} g \cdot u \mathrm{~d} \mathcal{H}^{1} .
$$

\subsection{The boundary conditions}

We suppose that $S$ is a bounded Lipschitz domain in $\mathbb{R}^{2}$ and we suppose that

$\Gamma \subset \partial S$ is a finite union of closed, disjoint, nontrivial intervals in $\partial S$.

Here, by an interval in $\partial S$ we mean a maximal connected set in $\partial S$. This condition is only needed for the construction of smooth approximations which preserve the boundary conditions on $\Gamma$. Note that in particular $\mathcal{H}^{1}(\Gamma)>0$. We fix

$$
\hat{u} \in W^{1, \infty}\left(S ; \mathbb{R}^{2}\right), \quad \hat{v} \in W^{2, \infty}(S) .
$$

For the three-dimensional problem we describe clamped boundary conditions by considering the admissible set

$$
\begin{aligned}
\mathcal{A}_{\Gamma}^{h}= & \left\{y^{(h)} \in W^{1,2}\left(\Omega ; \mathbb{R}^{3}\right): y^{(h)}\left(x^{\prime}, x_{3}\right)=\left(\begin{array}{c}
x^{\prime} \\
h x_{3}
\end{array}\right)+\left(\begin{array}{c}
h^{2} \hat{u}\left(x^{\prime}\right) \\
h \hat{v}\left(x^{\prime}\right)
\end{array}\right)-x_{3}\left(\begin{array}{cc}
h^{2} \nabla^{\prime} \hat{v} \\
0
\end{array}\right)\right. \\
& \text { for } \left.x^{\prime} \in \Gamma, x_{3} \in I\right\}
\end{aligned}
$$

where as before $I=(-1 / 2,1 / 2)$. For the limit problem we consider the admissible pairs

$$
\mathcal{A}_{\Gamma}=\left\{(u, v) \in W^{1,2}\left(S ; \mathbb{R}^{2}\right) \times W^{2,2}(S): u=\hat{u}, v=\hat{v}, \nabla^{\prime} v=\nabla^{\prime} \hat{v} \quad \text { on } \Gamma\right\} .
$$




\subsection{Normal forces}

We first consider the case of purely normal loading.

Theorem 1. Suppose $g^{(h)}=g=0$ and that the normal forces satisfy

$$
h^{-3} f^{(h)} \rightarrow f, \quad \text { in } L^{2}(S) \text {. }
$$

Let $y^{(h)} \in \mathcal{A}_{\Gamma}^{h}$ be a minimizing sequence for $J^{h}$ in the sense that

$$
h^{-4}\left(J^{h}\left(y^{(h)}\right)-\inf _{\mathcal{A}_{\Gamma}^{h}} J^{h}\right) \rightarrow 0 .
$$

Then

$$
\left(u^{(h)}, v^{(h)}\right) \rightarrow(u, v) \quad \text { in } W^{1,2}\left(S, \mathbb{R}^{3}\right) \text { and }(u, v) \in \mathcal{A}_{\Gamma} .
$$

Moreover $(u, v)$ minimizes $J^{\mathrm{vK}}$ in $\mathcal{A}_{\Gamma}$.

Remark 2. The conclusion that $(u, v) \in \mathcal{A}_{\Gamma}$ also holds if $y^{(h)}$ only satisfies the boundary conditions approximately. It suffices that the difference $Y^{(h)}=y^{(h)}-$ $\left(x^{\prime}, h x_{3}\right)$ satisfies

$$
\begin{gathered}
h^{-2} \int_{I}\left(\begin{array}{c}
Y_{1}^{(h)} \\
Y_{2}^{(h)}
\end{array}\right)\left(\cdot, x_{3}\right) \mathrm{d} x_{3} \rightarrow\left(\begin{array}{c}
\hat{u}_{1} \\
\hat{u}_{2}
\end{array}\right), \\
h^{-1} \int_{I} Y_{3}^{(h)}\left(\cdot, x_{3}\right) \mathrm{d} x_{3} \rightarrow \hat{v}, \\
h^{-2} \int_{I} x_{3} Y^{(h)}\left(\cdot, x_{3}\right) \mathrm{d} x_{3} \rightarrow-\frac{1}{12}\left(\begin{array}{c}
\nabla^{\prime} \hat{v} \\
0
\end{array}\right)
\end{gathered}
$$

in $L^{2}(\Gamma)$.

Remark 3. The assertion also holds for more general forces $f^{(h)}, f$ which may depend on $x_{3}$ and one can include additional forces $f_{ \pm}^{(h)}$ on the top and bottom surfaces $S \times\{ \pm 1 / 2\}$. Since the proof below still applies we focus on the simplest case.

\subsection{Normal and in-plane forces-the stability alternative}

The natural scaling for the in-plane forces is $g^{(h)} \sim h^{2}$. In the vK scaling the in-plane displacement scales as $h^{2} u$ and therefore the work done by the in-plane forces is of order $h^{4}$, which is consistent with the $\mathrm{vK}$ regime.

In-plane forces of order $h^{2}$ can, however, also lead to a very different regime, namely that of nonlinear bending theory (Kirchhoff-Love theory). This regime corresponds to a scaling of the elastic energy $e^{h}=\int W\left(\nabla_{h} y^{(h)}\right) \sim h^{2}$ and a displacement of order 1. Now the work done by the in-plane forces is of order $h^{2}$ which is again comparable to $e^{h}$. 
Also a third regime may arise, which corresponds to a geometrically linear version of the Kirchhoff-Love theory (in the sense of passing from (27) below to (28) and from $J^{\mathrm{Ki}}$ (see (26) below) to $J^{\mathrm{vK}}$, with $f=0$ ). This regime corresponds to an energy scaling $h^{4} \ll e^{h} \ll h^{2}$. tional

To give a precise statement of this alternative we introduce the Kirchhoff func-

$$
J^{\mathrm{Ki}}=\frac{1}{24} \int_{S} Q_{2}(A) \mathrm{d} x^{\prime}-\int_{\partial S} g \cdot\left(y^{\prime}-x^{\prime}\right) \mathrm{d} \mathcal{H}^{1},
$$

where the second fundamental form $A$ is given by $A_{\gamma \delta}=-y, \gamma \delta \cdot n$ with $n=y_{, 1} \wedge y_{, 2}$ and where $y$ belongs to the admissible class

$$
\begin{aligned}
\mathcal{A}_{\Gamma}^{\text {iso }}= & \left\{y \in W^{2,2}\left(S, \mathbb{R}^{3}\right)\right. \\
& \left.:\left(\nabla^{\prime} y\right)^{T} \nabla^{\prime} y=I d \text { in } S, y=\left(\begin{array}{c}
i d \\
0
\end{array}\right), \nabla^{\prime} y=\left(\begin{array}{c}
I d \\
0
\end{array}\right) \text { on } \Gamma\right\} .
\end{aligned}
$$

We also consider the geometrically linear version of this class, that is,

$$
\begin{aligned}
\mathcal{A}_{\Gamma}^{\text {iso,lin }}= & \left\{(u, v) \in W^{1,2}\left(S ; \mathbb{R}^{2}\right) \times W^{2,2}(S): 2 \operatorname{sym} \nabla u\right. \\
& \left.+\nabla v \otimes \nabla v=0 \text { in } S, u=v=\nabla^{\prime} v=0 \text { on } \Gamma\right\} .
\end{aligned}
$$

Theorem 4. Let $S$ be a bounded, simply connected domain with Lipschitz boundary and let $\Gamma \subset \partial S$ be as in (16). Suppose that

$$
h^{-3} f^{(h)} \rightarrow f \text { in } L^{2}(S), \quad h^{-2} g^{(h)} \rightarrow g \text { in } L^{2}\left(\partial S ; \mathbb{R}^{2}\right) .
$$

Let $y^{(h)} \in \mathcal{A}_{\Gamma}^{h}$ be a minimizing sequence for $J^{h}$ in the sense that

$$
h^{-4}\left(J^{h}\left(y^{(h)}\right)-\inf _{\mathcal{A}_{\Gamma}^{h}} J^{h}\right) \rightarrow 0 .
$$

Suppose that

(i) The Kirchhoff functional has no nontrivial minimizers, that is, $J^{\mathrm{Ki}}(y) \geqq 0$ for all $y \in \mathcal{A}_{\Gamma}^{\text {iso }}$ and equality only holds for $y\left(x^{\prime}\right)=\left(x^{\prime}, 0\right)$.

(ii) The constrained von Kármán functional (with $f=0$ ) has no nontrivial minimizers, that is, $J^{\mathrm{vK}}(u, v) \geqq 0$ for all $(u, v) \in \mathcal{A}_{\Gamma}^{\text {iso,lin }}$ and $J^{\mathrm{vK}}(u, v)=0$ for $(u, v) \in \mathcal{A}_{\Gamma}^{\text {iso,lin }}$ only if $(u, v)=0$.

Then the scaled displacements satisfy

$$
\left(u^{(h)}, v^{(h)}\right) \rightarrow(u, v) \quad \text { in } W^{1,2}\left(S, \mathbb{R}^{3}\right), \quad(u, v) \in \mathcal{A}_{\Gamma}
$$

and $(u, v)$ minimizes $J^{\mathrm{vK}}$ in $\mathcal{A}_{\Gamma}$.

In the example in Section 7 we will consider a boundary condition on one of the in-plane components only. In this case the stability alternative holds too. 
Corollary 5. Let $S=(0, L) \times(0,1), \Gamma=\{0\} \times(0,1), \Omega=S \times\left(-\frac{1}{2}, \frac{1}{2}\right)$. Set

$$
\begin{aligned}
\mathcal{B}_{\Gamma}^{h}= & \left\{y^{(h)} \in W^{1,2}\left(\Omega ; \mathbb{R}^{3}\right): y_{1}^{(h)}(x)=0, y_{3}^{(h)}(x)=h x_{3} \text { on } \Gamma \times(-1 / 2,1 / 2),\right. \\
& \left.\int_{\Gamma} x_{2} y_{2}^{(h)}(x) \mathrm{d} x_{2} \geqq 0, \int_{\Gamma} y_{2}^{(h)} \mathrm{d} x_{2}=0\right\}, \\
\mathcal{B}_{\Gamma}:= & \left\{(u, v) \in W^{1,2}\left(S, \mathbb{R}^{2}\right) \times W^{2,2}(S): u_{1}=v=\nabla^{\prime} v=0 \text { on } \Gamma, \int_{\Gamma} u_{2} \mathrm{~d} x_{2}=0\right\} .
\end{aligned}
$$

Then Theorem 4 remains valid if $\mathcal{A}_{\Gamma}^{h}$ and $\mathcal{A}_{\Gamma}$ are replaced with $\mathcal{B}_{\Gamma}^{h}$ and $\mathcal{B}_{\Gamma}$, respectively.

Note that in particular the definitions of $\mathcal{A}_{\Gamma}^{\text {iso }}$ and $\mathcal{A}_{\Gamma}^{\text {iso,lin }}$ are the same in Theorem 4 and Corollary 5 and involve boundary conditions for all components of $y$ and of $u$, respectively (see the proof of Corollary 5 for the details; roughly speaking, control of $y_{1}$ and $y_{3}$ on $\Gamma$ implies control of $y_{2}$, up to a possible 180 degree in-plane rotation). The inequality constraint in the definition of $\mathcal{B}_{\Gamma}^{h}$ is only introduced to rule out 180 degree in-plane rotations. It is automatically satisfied if $y^{(h)}$ is $L^{2}$ close to the trivial map $x \mapsto\left(x^{\prime}, 0\right)$. In view of Corollary 14 each low energy deformation (with $y_{1}^{(h)}=x_{1}$ and $y_{3}^{(h)}=h x_{3}$ ) is either close to the trivial map or to the map $x \mapsto\left(-x^{\prime}, 0\right)$. The equality constraint in $\mathcal{B}_{\Gamma}^{h}$ just removes the freedom of an arbitrary translation in $e_{2}$ direction.

\subsection{Nonlinear stability versus linearized stability}

In Section 6 we analyse the relationship between condition (i) (nonlinear stability) and condition (ii) (stability in the geometrically linear setting) in Theorem 4. For brevity we refer to condition (ii) as linearized stability, even though the underlying problem is nonlinear due to the constraint $\operatorname{det}\left(\nabla^{\prime}\right)^{2} v=0$ (which arises from the condition $2 \operatorname{sym} \nabla^{\prime} u+\nabla^{\prime} v \otimes \nabla^{\prime} v=0$, see Proposition 36 below). We show that the two stability notions are essentially equivalent if $\Gamma$, the part of $\partial S$ where the plate is clamped, is a single, nontrivial, interval. To emphasize the dependence on $g$ we temporarily write $J_{g}^{\mathrm{Ki}}$ for the functional in (26) and $J_{g}^{\mathrm{vK}}$ for the functional in (15) (with $f=0$ ).

Theorem 6. (Nonlinear stability implies geometrically linear stability) Let $S$ be a bounded, simply connected Lipschitz domain and suppose that $\Gamma$ is a compact, nontrivial interval. Suppose further that $J_{g}^{\mathrm{Ki}}(y) \geqq 0$ for all $y \in \mathcal{A}_{\Gamma}^{\mathrm{iso}}$. Then $J_{g}^{\mathrm{vK}}(u, v) \geqq$ 0 for all $(u, v) \in \mathcal{A}_{\Gamma}^{\text {iso,lin }}$. If $\varepsilon \in(0,1)$ then $J_{(1-\varepsilon) g}^{\mathrm{vK}}>0$ on $\mathcal{A}_{\Gamma}^{\text {iso,lin }} \backslash\{(0,0)\}$.

Theorem 7. (Linearized stability implies nonlinear stability) Let $S$ be a bounded, simply connected Lipschitz domain and suppose that $\Gamma \subset \partial S$ is a closed, nontrivial interval. Suppose that the quadratic form $Q_{2}$ is isotropic, that is, $Q_{2}(A)=\alpha|A|^{2}+$ $\beta(\operatorname{tr} A)^{2}$. Suppose that $J^{\mathrm{vK}} \geqq 0$ on $\mathcal{A}_{\Gamma}^{\text {iso,lin }}$ and if $J^{\mathrm{vK}}(u, v)=0$ then $(u, v)=0$. Then $J^{\mathrm{Ki}} \geqq 0$ on $\mathcal{A}_{\Gamma}^{\text {iso }}$ and $J^{\mathrm{Ki}}(y)=0$ for $y \in \mathcal{A}_{\Gamma}^{\text {iso }}$ only if $y\left(x^{\prime}\right)=\left(x^{\prime}, 0\right)$. 


\subsection{Main ingredients of the proof}

As in our earlier work one key ingredient is a quantitative rigidity estimate (or nonlinear Korn inequality) of the form

$$
\min _{R \in S O(3)}\|\nabla v-R\|_{L^{2}} \leqq C\|\operatorname{dist}(\nabla v, S O(3))\|_{L^{2}}
$$

This allows us to control the distance of a deformation from a rigid motion in terms of the elastic energy, see Theorems 8 and 9 below. For an elastic energy (per unit volume) of order $h^{4}$, one thus obtains bounds on the scaled displacements $u^{(h)}$ and $v^{(h)}$ as in [13], see Lemma 11. One new technical point is the use of the boundary conditions (18) to eliminate the freedom of a rigid motion, see Lemma 13. From this scaling one obtains a characterization of the limiting strain and a lower bound of the scaled nonlinear elastic energy by the vK functional, in the limit $h \rightarrow 0$, see Lemma 16 and Corollary 17. Together with a more or less standard comparison function (see (100)) and some careful approximation arguments this establishes the convergence results for purely normal forces.

The stability alternative is obtained similarly, but in this case one also has to study different rescalings of the displacements, corresponding to energies $e^{h}$ with $c h^{4} \leqq e^{h} \leqq C h^{2}$.

For the study of the relationship between linear and nonlinear stability we need another key ingredient: a careful analysis of the of isometric immersions $y: S \subset \mathbb{R}^{2} \rightarrow \mathbb{R}^{3}$ and their geometrically linear counterparts (defined by the condition $\left.\nabla^{\prime} u+\left(\nabla^{\prime} u\right)^{T}+\nabla^{\prime} v \otimes \nabla^{\prime} v=0\right)$. In particular we need precise criteria when such an isometric immersion can be reconstructed from its out-of-plane component and we study the sharp regularity properties of $W^{2,2}$ immersions (this class corresponds to finite bending energy). We use the fact that the usual properties of smooth developable surfaces still holds in the $W^{2,2}$ setting (a proof under even more general hypotheses was given by Pogorelov [34, Chapter II], [35, Chapter IX].) A shorter proof in the $W^{2,2}$ setting was recently given by PAKZAD [31], using ideas of KirchHeIM [21]. All these results are discussed in detail in Section 9.

\subsection{Related work}

The rigorous derivation of plate theories from three-dimensional nonlinear elasticity begins with the work of Le Dret and Raoult on membrane theory (which corresponds to the scaling $e^{h} \sim 1$ ) in the early 1990s [24-26] following work by ACERBI et al. for strings [1]. The rigorous justification of geometrically nonlinear bending theory, which was first proposed by KIRCHHOFF [22] and corresponds to $e^{h} \sim h^{2}$, was obtained only recently $[11,12,32,33]$. For the full picture of rigorous variational scaling limits (including the vK limit) and various open questions related to scalings $e^{h} \sim h^{\beta}$ with $1 \leqq \beta<2$ see [13], in particular Table 1. An independent justification of the vK equations (for small, smooth data and periodic boundary conditions) has recently been obtained by MonNEAU [28], through a clever application of the implicit function theorem. Earlier CIARLET [4] had obtained the vK equations through a formal asymptotic expansion. We do not discuss here the huge 
literature on derivation of lower-dimensional theories starting from geometrically linear three-dimensional elasticity (rigorous results go back at least to [29]; see [5] for an extensive literature review), since buckling and other instabilities strongly hinge on the geometrically nonlinear structure of elasticity. Very recently we learned about the very interesting work of GRABOVSKY and TRUSKINOVSKY [15]. They analyse the onset of buckling in terms of the second variation of the full elastic bodies. In contrast to us, they do not restrict attention to cylindrical bodies. Instead they allow arbitrary geometries and take the constant in Korn's inequality as a measure of slenderness (in our setting this constant scales like $h^{2}$ ). This allows them to derive universal asymptotic relations for the failure of strict positivity of the second variation, independent of a special geometry and the material law (the discussion is carried out in detail in a two-dimensional to one-dimensional setting, but their approach is largely dimension independent).

\subsection{Outline}

In Sections 3 and 4.1 we review the rigidity estimates and the estimates for the scaled displacements derived from it. In the rest of Section 4 we adapt these estimates to the situation with boundary conditions and show how they lead to a lower bound of the scaled three-dimensional energy by the vK functional.

In Section 5 we derive the convergence result for purely normal forces and the stability alternative for normal and in-plane forces. In Section 6 we establish the relations between nonlinear and linearized stability. As an illustration we discuss in detail the buckling of a rectangular plate in Section 7. In Section 8 we discuss a general relation between the linearized stability condition and the existence of minimizers for the vK functional. Finally in Section 9 we review some properties of isometric immersions in $W^{2,2}$.

\section{Geometric rigidity}

Theorem 8. (Quantitative rigidity estimates) Let $U$ be a bounded Lipschitz domain in $\mathbb{R}^{n}, n \geqq 2$. There exists a constant $C(U)$ with the following property. For each $v \in W^{1,2}\left(U, \mathbb{R}^{n}\right)$ there is an associated rotation $R \in \mathrm{SO}(\mathrm{n})$ such that,

$$
\|\nabla v-R\|_{L^{2}(U)} \leqq C(U)\|\operatorname{dist}(\nabla v, \mathrm{SO}(\mathrm{n}))\|_{L^{2}(U)} .
$$

The constant $C(U)$ can be chosen uniformly for a family of domains which are bilipschitz equivalent with controlled Lipschitz constants. The constant $C(U)$ is invariant under dilations.

For a proof and a discussion of the relation to results of JOHN $[18,19]$ and RESHETNYAK [36] see [12]. Other extensions of John's result were obtained by KOPYLOV [23] and EGOROV [7]. Kopylov has considered similar stability problems for almost solutions of a variety of partial differential equations.

In a thin domain $\Omega_{h}=S \times(-h / 2, h / 2)$ the constant $C\left(\Omega_{h}\right)$ degenerates like $h^{-2}$ (see (35) below). We can obtain a good approximation (at least in the interior) 
for $\nabla y$ by a piecewise constant map $R^{(h)}$ (with values in $S O(3)$ ) by covering $\Omega_{h}$ with cubes of size $h$. Application of Theorem 8 to two neighbouring cubes in addition yields a difference quotient estimate. Thus after mollification on a scale $h$ we can obtain another approximation $\tilde{R}^{(h)}$ (which in general no longer takes values exactly in $S O(3)$ ) whose gradient can be controlled in terms of the energy. This second approximation will prove useful to establish compactness and also higher regularity of the limits as $h \rightarrow 0$. The following result summarizes the estimates (up to the boundary) one can obtain in this way. As before we rescale to a fixed domain $\Omega$ and use the scaled gradient $\nabla_{h}=\left(\nabla^{\prime}, h^{-1} \partial_{3}\right)$.

Theorem 9. (Approximation by rotations in thin domains) Suppose that $S \subset \mathbb{R}^{2}$ is a Lipschitz domain and $\Omega=S \times\left(-\frac{1}{2}, \frac{1}{2}\right)$. Let $y \in W^{1,2}\left(\Omega ; \mathbb{R}^{3}\right)$ and

$$
E:=\int_{\Omega} \operatorname{dist}^{2}\left(\nabla_{h} y, S O(3)\right) \mathrm{d} x .
$$

Then there exist maps $R: S \rightarrow S O(3)$ and $\tilde{R}: S \rightarrow \mathbb{R}^{3 \times 3}$, with $|\tilde{R}| \leqq C$, $\tilde{R} \in W^{1,2}\left(S, \mathbb{R}^{3 \times 3}\right)$ such that

$$
\begin{gathered}
\left\|\nabla_{h} y-R\right\|_{L^{2}(\Omega)}^{2} \leqq C E, \quad\|R-\tilde{R}\|_{L^{2}(S)}^{2} \leqq C E, \\
\|\nabla \tilde{R}\|_{L^{2}(S)}^{2} \leqq \frac{C}{h^{2}} E, \quad\|R-\tilde{R}\|_{L^{\infty}(S)}^{2} \leqq \frac{C}{h^{2}} E .
\end{gathered}
$$

Moreover there exists a constant rotation $\bar{Q} \in S O(3)$ such that

$$
\left\|\nabla_{h} y-\bar{Q}\right\|_{L^{2}(\Omega)}^{2} \leqq \frac{C}{h^{2}} E .
$$

Here all constants depend only on $S$.

Proof. See [13].

For future reference we recall that Korn's inequality holds for Lipschitz domains (we will only need it for $S \subset \mathbb{R}^{2}$ and $p=2$ ).

Proposition 10. (Korn's inequality, [14]) Suppose that $\Omega \subset \mathbb{R}^{n}$ is a bounded Lipschitz domain and let $1<p<\infty$. Consider the space

$$
E^{p}(\Omega):=\left\{u \in L^{p}\left(\Omega ; \mathbb{R}^{n}\right): \operatorname{sym} \nabla u \in L^{p}\left(\Omega ; \mathbb{R}^{n \times n}\right)\right\}
$$

Then $E^{p}(\Omega)=W^{1, p}\left(\Omega ; \mathbb{R}^{n}\right)$ and

$$
\begin{aligned}
\|u\|_{1, p}^{p} & :=\int_{\Omega}|u|^{p}+|\nabla u|^{p} \mathrm{~d} x \leqq C_{p}(\Omega) \int_{\Omega}|u|^{p}+|\operatorname{sym} \nabla u|^{p} \mathrm{~d} x \\
\min & \left\{\|u-A x-b \mid\|_{1, p}^{p}: A+A^{T}=0, A \in R^{n \times n}, b \in \mathbb{R}^{n}\right\} \\
& \leqq C_{p}(\Omega) \int_{\Omega}|\operatorname{sym} \nabla u|^{p}
\end{aligned}
$$

If $\Gamma \subset \partial \Omega$ has positive $\mathcal{H}^{n-1}$ measure then

$$
\|u\|_{1, p}^{p} \leqq C_{p}(\Omega, \Gamma) \int_{\Omega}|\operatorname{sym} \nabla u|^{p}, \quad \text { for all } u \text { with } u_{\mid \Gamma}=0 .
$$


Proof. This follows from [14], Theorem 1 by the usual compactness argument; see, for example, [13] for the details.

\section{Scaling of in-plane and out-of-plane components and limiting strain}

\subsection{Results without boundary conditions}

It follows from Theorem 9 that for energies $E^{h}$ small compared to $h^{2}$ the deformation $y^{(h)}$ is close to the trivial map $\left(x^{\prime}, x_{3}\right) \mapsto\left(x^{\prime}, h x_{3}\right)$, up to a rigid motion. The following lemma provides detailed estimates for the difference between $y^{(h)}$ and the trivial deformation. In view of future applications it is convenient to consider a general sequence $E^{h}$. To avoid case distinctions we only consider the case $E^{h} \geqq h^{4}$ since this is sufficient in the following.

Lemma 11. (Convergence of scaled out-of-plane and in-plane deformations) Suppose that

$$
I^{h}\left(y^{(h)}\right) \leqq C E^{h}, \quad \lim _{h \rightarrow 0} h^{-2} E^{h}=0, \quad E^{h} \geqq h^{4} .
$$

Then there exists a subsequence of $y^{(h)}$, maps $\tilde{R}^{(h)}: S \rightarrow S O(3)$ and constants $\bar{R}^{(h)} \in S O(3), c^{(h)} \in \mathbb{R}^{3}$ such that $\tilde{y}^{(h)}:=\left(\bar{R}^{(h)}\right)^{T}\left(y^{(h)}-c^{(h)}\right)$, the in-plane and out-of-plane displacements

$$
\tilde{U}^{(h)}\left(x^{\prime}\right):=\int_{I}\left(\begin{array}{c}
\tilde{y}_{1}^{(h)} \\
\tilde{y}_{2}^{(h)}
\end{array}\right)\left(x^{\prime}, x_{3}\right)-\left(\begin{array}{l}
x_{1} \\
x_{2}
\end{array}\right) \mathrm{d} x_{3}, \quad \tilde{V}^{(h)}\left(x^{\prime}\right):=\int_{I} \tilde{y}_{3}^{(h)} \mathrm{d} x_{3}
$$

and the first moment

$$
\tilde{\zeta}^{(h)}\left(x^{\prime}\right)=\int_{I} x_{3}\left[\tilde{y}^{(h)}\left(x^{\prime}, x_{3}\right)-\left(\begin{array}{c}
x^{\prime} \\
h x_{3}
\end{array}\right)\right] \mathrm{d} x_{3}
$$

satisfy

$$
\begin{gathered}
\left\|\nabla_{h} \tilde{y}^{(h)}-\tilde{R}^{(h)}\right\|_{L^{2}(\Omega)} \leqq C \sqrt{E^{h}}, \\
\tilde{v}^{(h)}:=\frac{h}{\sqrt{E^{h}}} \tilde{V}^{(h)} \rightarrow \tilde{v} \quad \text { in } W^{1,2}(S), \quad \tilde{v} \in W^{2,2}(S), \\
\tilde{u}^{(h)}:=\frac{h^{2}}{E^{h}} \tilde{U}^{(h)} \rightarrow \tilde{u} \text { in } W^{1,2}\left(S ; \mathbb{R}^{2}\right), \\
\frac{1}{\sqrt{E^{h}}} \tilde{\zeta}^{(h)} \rightarrow-\frac{1}{12}\left(\begin{array}{c}
\nabla^{\prime} \tilde{v} \\
0
\end{array}\right) \text { in } W^{1,2}\left(S ; \mathbb{R}^{3}\right) .
\end{gathered}
$$

Proof. See [13].

Remark 12. The analogous assertion for $\tilde{\zeta}^{(h)}$ holds if $E^{h}=h^{2}$. Then $\nabla_{h} \tilde{y}^{(h)} \rightarrow \tilde{R}$ in $L^{2}$ and $h^{-1} \tilde{\zeta}^{(h)} \rightarrow(1 / 12)(\tilde{R}-I d) e_{3}$ in $W^{1,2}$; see [13]. 


\subsection{Clamped boundary conditions}

Later we will study maps $y^{(h)}$ which satisfy the boundary conditions

$$
y^{(h)}\left(x^{\prime}, x_{3}\right)=\left(\begin{array}{c}
x^{\prime} \\
h x_{3}
\end{array}\right)+\left(\begin{array}{c}
h^{2} \hat{u}\left(x^{\prime}\right) \\
h \hat{v}\left(x^{\prime}\right)
\end{array}\right)-x_{3}\left(\begin{array}{c}
h^{2} \nabla^{\prime} \hat{v} \\
0
\end{array}\right) \text { for } x^{\prime} \in \Gamma, x_{3} \in\left(-\frac{1}{2}, \frac{1}{2}\right) .
$$

Here $\Gamma \subset \partial S$ is a finite union of closed, disjoint, nontrivial intervals in $\partial S$ (so that in particular $\left.\mathcal{H}^{1}(\Gamma)>0\right)$ and $\hat{u} \in W^{1, \infty}\left(S ; \mathbb{R}^{2}\right)$ and $\hat{v} \in W^{2, \infty}(S)$. These boundary conditions break the rotational invariance and we will show that the scaling and convergence results in Lemma 11 hold already for $y^{(h)}$ (rather than $\tilde{y}^{(h)}$ ), that is, we may take $\bar{R}^{(h)}=I d$ and $c^{(h)}=0$, if we impose (45). In fact this assertion holds even if (45) is only satisfied approximately, that is, if suitably scaled quantities are bounded in $L^{2}(\Gamma)$.

We define the in-plane and out-of-plane displacements by

$$
U^{(h)}\left(x^{\prime}\right):=\int_{I}\left(\begin{array}{c}
y_{1}^{(h)} \\
y_{2}^{(h)}
\end{array}\right)\left(x^{\prime}, x_{3}\right)-\left(\begin{array}{l}
x_{1} \\
x_{2}
\end{array}\right) \mathrm{d} x_{3}, \quad V^{(h)}\left(x^{\prime}\right):=\int_{I} y_{3}^{(h)} \mathrm{d} x_{3} .
$$

and consider their scaled versions

$$
u^{(h)}=\frac{h^{2}}{E^{h}} U^{(h)}, \quad v^{(h)}=\frac{h}{\sqrt{E^{h}}} V^{(h)} .
$$

We also define the first moment by

$$
\zeta^{(h)}\left(x^{\prime}\right)=\int_{I} x_{3}\left[y^{(h)}\left(x^{\prime}, x_{3}\right)-\left(\begin{array}{c}
x^{\prime} \\
h x_{3}
\end{array}\right)\right] \mathrm{d} x_{3}
$$

Lemma 13. Suppose that

$$
I^{h}\left(y^{(h)}\right) \leqq C E^{h}, \quad \lim _{h \rightarrow 0} h^{-2} E^{h}=0, \quad E^{h} \geqq h^{4}
$$

and that

$$
\text { the traces } u^{(h)}, v^{(h)} \text {, and } \zeta^{(h)} / \sqrt{E^{h}} \text { are bounded in } L^{2}(\Gamma) \text {. }
$$

Then the assertions of Lemma 11 hold with $\tilde{y}^{(h)}, \tilde{u}^{(h)}, \tilde{v}^{(h)}, \tilde{R}^{(h)}$ replaced by $y^{(h)}$, $u^{(h)}, v^{(h)}, R^{(h)}$, where $R^{(h)}=\bar{R}^{(h)} \tilde{R}^{(h)}$. In other words, in Lemma 11 we may take $\bar{R}^{(h)}=I d$ and $c^{(h)}=0$. Specifically we have $\left\|\nabla_{h} y^{(h)}-R^{(h)}\right\|_{L^{2}} \leqq C \sqrt{E^{h}}$ and for a subsequence

$$
\begin{aligned}
& v^{(h)} \rightarrow v \text { in } W^{1,2}(S), \quad u^{(h)} \rightarrow u \text { in } W^{1,2}\left(S ; \mathbb{R}^{2}\right), \\
& \frac{1}{\sqrt{E^{h}}} \zeta^{(h)} \rightarrow-\frac{1}{12}\left(\begin{array}{c}
\nabla^{\prime} v \\
0
\end{array}\right) \text { in } W^{1,2}\left(S ; \mathbb{R}^{3}\right),
\end{aligned}
$$

with $v \in W^{2,2}(S)$. 
Proof. The main point is to show that the boundary conditions imply that $\bar{R}^{(h)}$ must be close to the identity. We claim that

$$
\bar{R}^{(h)}=I d+\frac{\sqrt{E^{h}}}{h}\left(e_{3} \otimes \bar{a}^{(h)}-\bar{a}^{(h)} \otimes e_{3}\right)+\mathcal{O}\left(\frac{E^{h}}{h^{2}}\right), \quad\left|\bar{a}^{(h)}\right| \leqq C .
$$

Using the relation $y^{(h)}=\bar{R}^{(h)} \tilde{y}^{(h)}+c^{(h)}$ we see that

$$
\begin{aligned}
\left(\begin{array}{c}
h^{-2} E^{h} u^{(h)} \\
h^{-1} \sqrt{E^{h}} v^{(h)}
\end{array}\right) & =\left(\bar{R}^{(h)}-I d\right)\left(\begin{array}{c}
x^{\prime} \\
0
\end{array}\right)+\bar{R}^{(h)}\left(\begin{array}{c}
h^{-2} E^{h} \tilde{u}^{(h)} \\
h^{-1} \sqrt{E^{h}} \tilde{v}^{(h)}
\end{array}\right)+c^{(h)}, \\
\zeta^{(h)} & =\left(\bar{R}^{(h)}-I d\right) \frac{1}{12} h e_{3}+\bar{R}^{(h)} \tilde{\zeta}^{(h)} .
\end{aligned}
$$

From (44), (55), the embedding $W^{1,2}(S) \hookrightarrow L^{2}(\Gamma)$ and the assumed bound on $\zeta^{(h)}$ in $L^{2}(\Gamma)$ we see that

$$
\left|\left(\bar{R}^{(h)}-I d\right) e_{3}\right| \leqq C h^{-1} \sqrt{E^{h}}
$$

Since $R^{(h)} \in S O(3)$ this implies that

$$
\left|\left(\left[\bar{R}^{(h)}\right]^{T}-I d\right) e_{3}\right| \leqq C h^{-1} \sqrt{E^{h}} .
$$

Let $\bar{Q}^{(h)}$ denote the $2 \times 2$ submatrix of $\bar{R}^{(h)}$ with entries $\bar{R}_{\gamma \delta}^{(h)}, \gamma, \delta \in\{1,2\}$. Then (56) and (57) imply that there exists $\hat{Q}^{(h)} \in S O(2)$ with

$$
\left|\bar{Q}^{(h)}-\hat{Q}^{(h)}\right| \leqq C h^{-2} E^{h} .
$$

Now consider the in-plane component of (54). Using (56), (58), the assumption on $u^{(h)}$ and the bounds (42) and (43) on $\tilde{u}^{(h)}$ and $\tilde{v}^{(h)}$ in combination with the embedding $W^{1,2}(S) \hookrightarrow L^{2}(\Gamma)$ we obtain

$$
\left\|\left(\hat{Q}^{(h)}-I d\right) x^{\prime}+\left(\begin{array}{c}
c_{1}^{(h)} \\
c_{2}^{(h)}
\end{array}\right)\right\|_{L^{2}(\Gamma)} \leqq C h^{-2} E^{h} .
$$

After a possible translation of $S$ and a corresponding adjustment of the constants $c_{\gamma}^{(h)}, \gamma \in\{1,2\}$ we may assume that $\int_{\Gamma} x^{\prime} \mathrm{d} \mathcal{H}^{1}=0$. Now every matrix $Q \in S O(2)$ satisfies

$$
2\left|(Q-I d) x^{\prime}\right|^{2}=|Q-I d|^{2}\left|x^{\prime}\right|^{2} .
$$

Since

$$
\int_{\Gamma}\left|x^{\prime}\right|^{2} \mathrm{~d} \mathcal{H}^{1}>0
$$

by expanding the left-hand side of (59) we deduce that

$$
\left|\hat{Q}^{(h)}-I d\right|^{2} \leqq C h^{-2} E^{h}
$$


and subsequently

$$
\left|c_{\gamma}^{(h)}\right| \leqq C h^{-2} E^{h}, \quad \text { for } \gamma \in\{1,2\}
$$

Combining (56), (57) and (62) we see that $\left|\bar{R}^{(h)}-I d\right| \leqq C h^{-1} \sqrt{E^{h}}$. Thus

$$
2 \operatorname{sym}\left(\bar{R}^{(h)}-I d\right)=-\left(\left(\bar{R}^{(h)}\right)^{T}-I d\right)\left(\bar{R}^{(h)}-I d\right)
$$

is of order $h^{-2} E^{h}$. Together with (58) and (62) this establishes the desired representation (53). From the out-of-plane component of (54) we see that $\left|c_{3}^{(h)}\right| \leqq$ $C h^{-1} \sqrt{E^{h}}$.

Together with (54) we now easily deduce (for a subsequence) (51). From the out-of-plane component of (54) the representation (53) we see that $v=\bar{a} \cdot x^{\prime}+\tilde{v}+\bar{c}$. Thus $v \in W^{2,2}$ and $\nabla^{\prime} v=\bar{a}+\nabla^{\prime} \tilde{v}$. Together with (44), (53) and (55) and this proves (52).

A similar result holds if we control only one component of $u$. This will be useful when we discuss the example of buckling of a rectangular plate below.

Corollary 14. Suppose that

$$
I^{h}\left(y^{(h)}\right) \leqq C E^{h}, \quad \lim _{h \rightarrow 0} h^{-2} E^{h}=0, \quad E^{h} \geqq h^{4}
$$

and that

the traces $u_{1}^{(h)}, v^{(h)}$, and $\zeta^{(h)} / \sqrt{E^{h}}$ are bounded in $L^{2}(\Gamma)$.

(i) If $\Gamma$ is not contained in a straight line, then the assertions of Lemma 11 hold with $\tilde{y}^{(h)}, \tilde{u}^{(h)}, \tilde{v}^{(h)}, \tilde{R}^{(h)}$ replaced by $y^{(h)}, u^{(h)}, v^{(h)}, R^{(h)}$, where $R^{(h)}=$ $\bar{R}^{(h)} \tilde{R}^{(h)}$. In other words, in Lemma 11 we may take $\bar{R}^{(h)}=$ Id and $c^{(h)}=0$ and (51) and (52) hold.

(ii) If $\Gamma$ is contained in a line with direction $\tau=\left(\begin{array}{l}\cos \alpha \\ \sin \alpha\end{array}\right)$ and $0<\alpha<\pi$ then the assertion of Lemma 11 hold with

$$
\bar{R}^{(h)}=I d \text { or with } \bar{R}^{(h)}=\left(\begin{array}{ccc}
\cos \theta & -\sin \theta & 0 \\
\sin \theta & \cos \theta & 0 \\
0 & 0 & 1
\end{array}\right), \quad \theta=2 \pi-2 \alpha .
$$

Proof. The argument up to and including (58) is unchanged. Taking the first component of (54) and using the boundedness of $u_{1}^{(h)}$ in $L^{2}(\Gamma)$ we deduce that

$$
\left\|e_{1} \cdot\left(\hat{Q}^{(h)}-I d\right) x^{\prime}+c_{1}^{(h)}\right\|_{L^{2}(\Gamma)} \leqq h^{-2} E^{h} .
$$

Again we may suppose without loss of generality that $\int_{\Gamma} x^{\prime}=0$. Then expansion of the previous expression yields $\left|c_{1}^{(h)}\right| \leqq h^{-2} E^{h}$ and

$$
\left|a \otimes a: \int_{\Gamma} x^{\prime} \otimes x^{\prime} \mathrm{d} \mathcal{H}^{1}\right|^{1 / 2} \leqq C h^{-2} E^{h}, \quad \text { where } a=\left(\left(\hat{Q}^{h}\right)^{T}-I d\right) e_{1} .
$$


If $\Gamma$ is not contained in a straight line then $\int_{\Gamma} x^{\prime} \otimes x^{\prime}$ is a matrix of full rank and we get $|a| \leqq C h^{-2} E^{h}$. As in (60) we deduce that $\left|\hat{Q}^{(h)}-I d\right| \leqq C h^{-2} E^{h}$ and the proof can be finished as before.

Now suppose that $\Gamma$ is contained in a line with direction $\tau=\left(\begin{array}{c}\cos \alpha \\ \sin \alpha\end{array}\right)$ and write

$$
\hat{Q}^{(h)}=\left(\begin{array}{cc}
\cos \theta^{(h)} & -\sin \theta^{(h)} \\
\sin \theta^{(h)} & \cos \theta^{(h)}
\end{array}\right) .
$$

Then the estimate (69) yields

$$
\left|\left(\begin{array}{c}
\cos \alpha \\
\sin \alpha
\end{array}\right) \cdot\left(\left(\begin{array}{c}
\cos \theta^{(h)} \\
-\sin \theta^{(h)}
\end{array}\right)-e_{1}\right)\right| \leqq C h^{-2} E^{h}
$$

and this implies that $\left|\cos \left(\alpha+\theta^{(h)}\right)-\cos \alpha\right| \leqq C h^{-2} E^{h}$. Since $\alpha$ is different from 0 and $\pi$ we deduce that either $\theta^{(h)}=\mathcal{O}\left(h^{-2} E^{h}\right)$ or $\theta^{(h)}=2 \pi-2 \alpha+\mathcal{O}\left(h^{-2} E^{h}\right)$. This finishes the proof of Corollary 14.

\subsection{Clamped boundary conditions for the out-of-plane component}

We now consider the situation where instead of the full boundary data as in (45) we only have control of the out-of-plane component $v$ and its derivative $\nabla^{\prime} v$. In this case we still have the freedom of an in-plane rotation and translation and the following result shows that and the scaling and convergence results in Lemma 11 hold up to this restricted invariance.

Lemma 15. Suppose that

$$
I^{h}\left(y^{(h)}\right) \leqq C E^{h}, \quad \lim _{h \rightarrow 0} h^{-2} E^{h}=0, \quad E^{h} \geqq h^{4}
$$

and that

$$
\text { the traces } v^{(h)} \text { and } \zeta^{(h)} / \sqrt{E^{h}} \text { are bounded in } L^{2}(\Gamma) \text {. }
$$

Then in Lemma 11 we may choose $\bar{R}^{(h)}$ as an in-plane rotation and $c^{(h)}$ and an in-plane translation, that is,

$$
\bar{R}^{(h)}=\left(\begin{array}{cc}
\hat{Q}^{(h)} & 0 \\
0 & 1
\end{array}\right), \quad c_{3}^{(h)}=0 .
$$

Proof. The proof is very similar to the one for full boundary conditions. We still have (54) and (55). From (55) we obtain, as in the proof of Lemma 13, the estimates (56)-(58). Using the out-of-plane component of (54) in connection with (57) and the weak convergence of $\tilde{u}^{(h)}$ and $\tilde{v}^{(h)}$ we obtain

$$
\left|c_{3}^{(h)}\right| \leqq C h^{-1} \sqrt{E^{h}}
$$

We now set

$$
\hat{y}^{(h)}:=\left(\begin{array}{cc}
\hat{Q}^{(h)} & 0 \\
0 & 1
\end{array}\right)^{T}\left[y^{(h)}-\left(\begin{array}{c}
c^{(h)} \\
0
\end{array}\right)\right], \quad c^{(h)}=\left(\begin{array}{c}
c_{1}^{(h)} \\
c_{2}^{(h)}
\end{array}\right),
$$


where $\hat{Q}^{(h)} \in S O(2)$ is the matrix in (58). Then

$$
\hat{y}^{(h)}=\bar{S}^{(h)} \tilde{y}^{(h)}+\left(\begin{array}{c}
0 \\
(h)
\end{array}\right)
$$

where

$$
\bar{S}^{(h)}:=\left(\begin{array}{cc}
\hat{Q}^{(h)} & 0 \\
0 & 1
\end{array}\right)^{T} \bar{R}^{(h)}
$$

We also set

$$
\hat{R}^{(h)}:=\bar{S}^{(h)} \tilde{R}^{(h)}
$$

We define $\hat{u}^{(h)}, \hat{v}^{(h)}$ and $\hat{\zeta}^{(h)}$ in the usual way on the basis of $\hat{y}^{(h)}$. To prove Lemma 15 we show that the assertions of Lemma 11 hold with $\tilde{y}^{(h)}, \tilde{u}^{(h)}, \tilde{v}^{(h)}$, etc. replaced by $\hat{y}^{(h)}, \hat{u}^{(h)}, \hat{v}^{(h)}$, etc.

We see from (56)-(58) that

$$
\begin{gathered}
\left|\left(\bar{S}^{(h)}-I d\right)_{\gamma \delta}\right| \leqq C h^{-2} E^{h}, \quad \text { for } \gamma, \delta \in\{1,2\}, \\
\left|\left(\bar{S}^{(h)}-I d\right)_{3 j}\right|+\left|\left(\bar{S}^{(h)}-I d\right)_{j 3}\right| \leqq C h^{-1} \sqrt{E^{h}}, \quad \text { for } j \in\{1,2,3\} .
\end{gathered}
$$

Now for every $S \in S O(3)$ we have $2 \operatorname{sym}(S-I d)=-(S-I d)^{T}(S-I d)$ and this shows that

$$
\bar{S}^{(h)}=I d+\frac{\sqrt{E^{h}}}{h}\left(e_{3} \otimes a^{(h)}-a^{(h)} \otimes e_{3}\right)+\mathcal{O}\left(\frac{E^{h}}{h^{2}}\right), \quad a^{(h)} \in \mathbb{R}^{2}, \quad\left|a^{(h)}\right| \leqq C .
$$

Since $\bar{S}^{(h)} \in S O(3)$ we immediately deduce from (74) and (75) that $\mid \nabla_{h} \hat{y}^{(h)}-$ $\hat{R}^{(h)}|=| \nabla_{h} \tilde{y}^{(h)}-\tilde{R}^{(h)} \mid$. Thus (41) holds for $\hat{y}^{(h)}$ and $\hat{R}^{(h)}$. Moreover

$$
\begin{gathered}
\left(\begin{array}{c}
h^{-2} E^{h} \hat{u}^{(h)} \\
h^{-1} \sqrt{E^{h}} v^{(h)}
\end{array}\right)=\left(\bar{S}^{(h)}-I d\right)\left(\begin{array}{c}
x^{\prime} \\
0
\end{array}\right)+\bar{S}^{(h)}\left(\begin{array}{c}
h^{-2} E^{h} \tilde{u}^{(h)} \\
h^{-1} \sqrt{E^{h}} \tilde{v}^{(h)}
\end{array}\right)+\left(\begin{array}{c}
0 \\
c_{3}^{(h)}
\end{array}\right), \\
\hat{\zeta}^{(h)}=\left(\bar{S}^{(h)}-I d\right) \frac{1}{12} h e_{3}+\bar{S}^{(h)} \tilde{\zeta}^{(h)}
\end{gathered}
$$

From the bounds (78) for $\bar{S}^{(h)}$ and (73) for $c_{3}^{(h)}$ and the convergence results (43) and (42) for $\tilde{u}^{(h)}$ and $\tilde{v}^{(h)}$ we immediately deduce the corresponding convergence results for $\hat{u}^{(h)}$ and $\hat{v}^{(h)}$ (for a subsequence). As before we see from (78) that $\hat{v} \in W^{2,2}$ and $\nabla^{\prime} \hat{v}=\bar{a}+\nabla^{\prime} \tilde{v}$. This shows that $\hat{\zeta}^{(h)}$ converges to the right limit. 


\subsection{Identification of the limiting strain}

We know that $\nabla_{h} y^{(h)}$ can be well approximated by rotations $R^{(h)}\left(x^{\prime}\right)$. Since $W$ is invariant under rotations, the energy of $y^{(h)}$ is essentially controlled by the deviation of $\left(R^{(h)}\right)^{T} \nabla_{h} y^{(h)}$ from the identity. In view of (41) the quantities $G^{(h)}:=$ $\left(1 / \sqrt{E^{h}}\right)\left[\left(R^{(h)}\right)^{T} \nabla_{h} y^{(h)}-I d\right]$ converge weakly in $L^{2}$ (for a subsequence) to $G$. The following lemma shows that the relevant part of $G$ (that is, the symmetric part of the in-plane components) can be identified in terms of $u$ and $v$, the limits of the scaled in-plane and out-of-plane displacements. In particular, we show that the relevant components of $G$ are affine in the thickness variable $x_{3}$, a fact which is often assumed $a$ priori. The representation of $G$ immediately yields the lower bound in the definition of $\Gamma$ convergence (see the corollary immediately following the lemma).

Lemma 16. (Identification of scaled limiting strain) Consider $y^{(h)}: \Omega \rightarrow \mathbb{R}^{3}$ and $R^{(h)}: S \rightarrow S O(3)$ and define $u^{(h)}, v^{(h)}$ as in (46), (47). Suppose that

$$
\begin{gathered}
I^{h}\left(y^{(h)}\right) \leqq C E^{h}, \quad \lim _{h \rightarrow 0} h^{-2} E^{h}=0, \quad E^{h} \geqq h^{4}, \\
\left\|\nabla_{h} y^{(h)}-R^{(h)}\right\|_{L^{2}(\Omega)} \leqq C \sqrt{E^{h}}, \\
u^{(h)} \rightarrow u \text { in } W^{1,2}\left(S ; \mathbb{R}^{2}\right), \quad v^{(h)} \rightarrow v \text { in } W^{1,2}\left(S ; \mathbb{R}^{2}\right), \quad v \in W^{2,2}(S) .
\end{gathered}
$$

Then

$$
\frac{h}{\sqrt{E^{h}}}\left(R^{(h)}-I d\right) \rightarrow A=e_{3} \otimes \nabla^{\prime} v-\nabla^{\prime} v \otimes e_{3} \quad \text { in } L^{2}\left(S ; \mathbb{R}^{3 \times 3}\right) .
$$

Moreover

$$
G^{(h)}:=\frac{\left(R^{(h)}\right)^{T} \nabla_{h} y^{(h)}-I d}{\sqrt{E^{h}}} \rightarrow G \text { in } L^{2}\left(\Omega ; \mathbb{R}^{3 \times 3}\right)
$$

and the $2 \times 2$ submatrix $G^{\prime \prime}$ given by $G_{\alpha \beta}^{\prime \prime}=G_{\alpha \beta}$ for $1 \leqq \alpha, \beta \leqq 2$ satisfies

$$
G^{\prime \prime}\left(x^{\prime}, x_{3}\right)=G_{0}\left(x^{\prime}\right)+x_{3} G_{1}\left(x^{\prime}\right), \quad G_{1}=-\left(\nabla^{\prime}\right)^{2} v .
$$

We have

$$
\begin{gathered}
2 \operatorname{sym} \nabla^{\prime} u+\nabla^{\prime} v \otimes \nabla^{\prime} v=0, \quad \text { if } h^{-4} E^{h} \rightarrow \infty, \\
\operatorname{sym} G_{0}=\operatorname{sym} \nabla^{\prime} u+\frac{1}{2} \nabla^{\prime} v \otimes \nabla^{\prime} v, \quad \text { if } h^{-4} E^{h} \rightarrow 1 .
\end{gathered}
$$

Proof. See [13].

Corollary 17. Let $E^{h}, y^{(h)}, R^{(h)}, u^{(h)}, v^{(h)}$ be as in the lemma above.

(i) If $\lim _{h \rightarrow 0} h^{-4} E^{h}=\infty$ then

$$
\liminf _{h \rightarrow 0} \frac{1}{E^{h}} I^{h}\left(y^{(h)}\right) \geqq \int_{S} \frac{1}{24} Q_{2}\left(\left(\nabla^{\prime}\right)^{2} v\right) \mathrm{d} x^{\prime} .
$$


(ii) If $\lim _{h \rightarrow 0} h^{-4} E^{h}=1$ then

$$
\begin{aligned}
\liminf _{h \rightarrow 0} \frac{1}{E^{h}} I^{h}\left(y^{(h)}\right) \geqq & \left.\int_{S} \frac{1}{2} Q_{2}\left(\operatorname{sym} \nabla^{\prime} u+\frac{1}{2} \nabla^{\prime} v \otimes \nabla^{\prime} v\right]\right) \\
& +\frac{1}{24} \int_{S} Q_{2}\left(\left(\nabla^{\prime}\right)^{2} v\right) \mathrm{d} x^{\prime} .
\end{aligned}
$$

Proof. See [13].

Remark 18. Lemma 16 and Corollary 17 can be applied to the quadruple $\left(y^{(h)}\right.$, $\left.R^{(h)}, u^{(h)}, v^{(h)}\right)$ in Lemma 13 as well as to the quadruple $\left(\tilde{y}^{(h)}, \tilde{R}^{(h)}, \tilde{u}^{(h)}, \tilde{v}^{(h)}\right)$ in Lemma 11.

\section{Derivation and range of validity of the vK theory}

In this section we prove Theorems 1 (convergence for purely normal forces) and 4 (stability alternative for in-plane plus normal forces).

\subsection{Normal forces}

Proof of Theorem 1. The argument is similar to the case without boundary conditions treated in [13]. The main point is that the boundary conditions allow us to eliminate the $h$-dependent rigid motions.

Step 1 (a priori bounds for $J^{h}\left(y^{(h)}\right)$ and $\left.I^{h}\left(y^{(h)}\right)\right)$. Using the test function

$$
\check{y}\left(x^{\prime}, x_{3}\right)=\left(\begin{array}{c}
x^{\prime} \\
h x_{3}
\end{array}\right)+\left(\begin{array}{c}
h^{2} \hat{u}\left(x^{\prime}\right) \\
h \hat{v}\left(x^{\prime}\right)
\end{array}\right)-x_{3}\left(\begin{array}{c}
h^{2} \nabla^{\prime} \hat{v} \\
0
\end{array}\right)
$$

we see that inf ${ }_{\mathcal{A}_{\Gamma}^{h}} J^{h} \leqq C h^{4}$. We claim that

$$
e^{h}:=I^{h}\left(y^{(h)}\right) \leqq C h^{4} .
$$

It follows from (35) that there exists an affine function

$$
l^{(h)}(x)=\bar{Q}^{(h)} x+c^{(h)}, \quad \bar{Q}^{(h)} \in S O(3), \quad c^{(h)} \in \mathbb{R}^{3}
$$

such that

$$
\left\|y^{(h)}-l^{(h)}\right\|_{W^{1,2}(\Omega)} \leqq C h^{-1} \sqrt{e^{h}} .
$$

Using the embedding $W^{1,2}(S) \hookrightarrow L^{2}(\Gamma)$ we deduce that

$$
\begin{gathered}
\left\|\int_{I} y_{3}^{(h)}\left(\cdot, x_{3}\right) \mathrm{d} x_{3}-\int_{I} l_{3}^{(h)}\left(\cdot, x_{3}\right) \mathrm{d} x_{3}\right\|_{L^{2}(\Gamma)} \\
\| C h^{-1} \sqrt{e^{h}} \\
\left\|\int_{I} x_{3} y_{\gamma}^{(h)}\left(\cdot, x_{3}\right) \mathrm{d} x_{3}-\int_{I} x_{3} l_{\gamma}^{(h)}\left(\cdot, x_{3}\right) \mathrm{d} x_{3}\right\|_{L^{2}(\Gamma)} \leqq C h^{-1} \sqrt{e^{h}}, \quad \gamma \in\{1,2\} .
\end{gathered}
$$


Taking into account the boundary conditions for $y^{(h)}$ and the relation $Q_{13}^{2}+Q_{23}^{2}=$ $Q_{31}^{2}+Q_{32}^{2}$, which is valid for every $Q \in S O(3)$, we easily deduce that $\left|c_{3}^{(h)}\right|+$ $\left|\bar{Q}_{31}^{(h)}\right|+\left|\bar{Q}_{32}^{(h)}\right| \leqq C h^{-1} \sqrt{e^{h}}+C h$ and thus

$$
\left\|y_{3}^{(h)}\right\|_{W^{1,2}(\Omega)} \leqq C h^{-1} \sqrt{e^{h}}+C h .
$$

Hence

$$
e^{h}=J^{h}\left(y^{(h)}\right)+\int_{\Omega} f_{3}^{(h)} y_{3}^{(h)} \leqq C h^{2} \sqrt{e^{h}}+C h^{4}
$$

and this proves the claim (90).

Step 2 (convergence of $u^{(h)}$ and $\left.v^{(h)}\right)$. In view of Step 1 we can apply Lemma 13 with $E^{h}=h^{4}$. This yields

$$
u^{(h)} \rightarrow u, \quad v^{(h)} \rightarrow v, \quad \frac{1}{h^{2}} \zeta^{(h)} \rightarrow-\frac{1}{12}\left(\begin{array}{c}
\nabla^{\prime} v \\
0
\end{array}\right)
$$

in $W^{1,2}$. The compact embedding $W^{1,2}(S) \hookrightarrow L^{2}(\Gamma)$ in connection with the boundary conditions in (18) implies that $(u, v) \in \mathcal{A}_{\Gamma}$.

Step 3 (lower bound for $J^{h}\left(y^{(h)}\right)$ ). We claim that

$$
\liminf _{h \rightarrow 0} h^{-4} J^{h}\left(y^{(h)}\right) \geqq J^{\mathrm{vK}}(u, v),
$$

where $(u, v)$ is the limit of $\left(u^{(h)}, v^{(h)}\right)$. Indeed by Lemma 16 and Corollary 17 with $E^{h}=h^{4}$ we have

$$
\liminf _{h \rightarrow 0} h^{-4} I^{h}\left(y^{(h)}\right) \geqq I^{\mathrm{vK}}(u, v) .
$$

Moreover

$$
h^{-4} \int_{\Omega} f_{3}^{(h)} y_{3}^{(h)} \mathrm{d} x=\int_{S} h^{-3} f_{3}^{(h)} v^{(h)} \mathrm{d} x^{\prime} \rightarrow \int_{S} f_{3} v \mathrm{~d} x^{\prime} .
$$

Thus (94) holds.

Step 4 (upper bound, convergence of energy and minimizing property of $(u, v)$ ).

Let $(\breve{u}, \breve{v}) \in \mathcal{A}_{\Gamma}$. We claim that there exist $\check{y}^{(h)} \in \mathcal{A}_{\Gamma}^{h}$ such that

$$
\begin{gathered}
\frac{1}{h^{2}} \int_{I}\left(\begin{array}{l}
\check{y}_{1}^{(h)} \\
\check{y}_{2}^{(h)}
\end{array}\right)-\left(\begin{array}{l}
x_{1} \\
x_{2}
\end{array}\right) \mathrm{d} x_{3} \rightarrow \check{u} \quad \text { in } W^{1,2}\left(S ; \mathbb{R}^{2}\right), \\
\frac{1}{h} \int_{I} \check{y}_{3}^{(h)} \mathrm{d} x_{3} \rightarrow \check{v} \quad \text { in } W^{1,2}(S) .
\end{gathered}
$$

and

$$
h^{-4} J^{h}\left(\check{y}^{(h)}\right) \rightarrow J^{\mathrm{vK}}(\check{u}, \check{v}) .
$$


Once this is shown the argument is easily finished. Indeed we have by the almost minimizing property of $y^{(h)}$ and Step 3

$$
\begin{aligned}
J^{\mathrm{vK}}(u, v) & \leqq \liminf _{h \rightarrow 0} h^{-4} J^{h}\left(y^{(h)}\right) \\
& \leqq \limsup _{h \rightarrow 0} h^{-4} J^{h}\left(y^{(h)}\right) \leqq \limsup _{h \rightarrow 0} h^{-4} J^{h}\left(\check{y}^{(h)}\right) \\
& =J^{\mathrm{vK}}(\check{u}, \check{v}) .
\end{aligned}
$$

Thus $(u, v)$ minimizes $J^{\mathrm{vK}}$ in $\mathcal{A}_{\Gamma}$. Taking $(\check{u}, \check{v})=(u, v)$ in the above chain of inequalities we obtain

$$
\lim _{h \rightarrow 0} h^{-4} J^{h}\left(y^{(h)}\right)=J^{\mathrm{vK}}(u, v) .
$$

Using this convergence in energy we can improve weak convergence of $u^{(h)}$ to strong convergence, that is,

$$
u^{(h)} \rightarrow u \text { in } W^{1,2}\left(S ; \mathbb{R}^{2}\right),
$$

see Section 7 in [13] for the proof.

This finishes the proof or Theorem 1, up to the verification of (97). To simplify the notation we write $u, v, y^{(h)}$ instead of $\breve{u}, \check{v}, \breve{y}^{(h)}$. The construction of $y^{(h)}$ is essentially the same as in [13], we only have to take care of the boundary conditions.

We assume first that $u$ and $v$ are smooth and as in [13] we consider the ansatz

$$
\hat{y}^{(h)}\left(x^{\prime}, x_{3}\right)=\left(\begin{array}{c}
x^{\prime} \\
h x_{3}
\end{array}\right)+\left(\begin{array}{c}
h^{2} u \\
h v
\end{array}\right)-h^{2} x_{3}\left(\begin{array}{c}
v_{, 1} \\
v_{, 2} \\
0
\end{array}\right)+h^{3} x_{3} d^{(0)}+\frac{h^{3}}{2} x_{3}^{2} d^{(1)},
$$

This ansatz only satisfies the boundary conditions up to higher order terms but we will resolve that difficulty later. We have

$$
\begin{aligned}
& \nabla_{h} \hat{y}^{(h)}=I d+\left(\begin{array}{c|c}
h^{2} \nabla^{\prime} u & -h\left(\nabla^{\prime} v\right)^{T} \\
\hline h \nabla^{\prime} v & 0
\end{array}\right)-h^{2} x_{3}\left(\begin{array}{c|c}
\left(\nabla^{\prime}\right)^{2} v & 0 \\
\hline 0 & 0
\end{array}\right) \\
& +h^{2} d^{(0)} \otimes e_{3}+h^{2} x_{3} d^{(1)} \otimes e_{3}+\mathcal{O}\left(h^{3}\right)
\end{aligned}
$$

Using the identities $(I+A)^{T}(I+A)=I+2 \operatorname{sym} A+A^{T} A$ and $\left(e_{3} \otimes a^{\prime}-a^{\prime} \times\right.$ $\left.e_{3}\right)^{T}\left(e_{3} \otimes a^{\prime}-a^{\prime} \times e_{3}\right)=a^{\prime} \otimes a^{\prime}+\left|a^{\prime}\right|^{2} e_{3} \otimes e_{3}$ for $a^{\prime} \in \mathbb{R}^{2}$ we obtain for the nonlinear strain

$$
\begin{aligned}
\left(\nabla_{h} \hat{y}^{(h)}\right)^{T} \nabla_{h} \hat{y}^{(h)}= & I d+2 h^{2}\left(\operatorname{sym} \nabla^{\prime} u-x_{3}\left(\nabla^{\prime}\right)^{2} v\right) \\
& +h^{2}\left(\nabla^{\prime} v \otimes \nabla^{\prime} v+\left|\nabla^{\prime} v\right|^{2} e_{3} \otimes e_{3}\right) \\
& +2 h^{2} \operatorname{sym}\left[\left(d^{(0)}+x_{3} d^{(1)}\right) \otimes e_{3}\right]+\mathcal{O}\left(h^{3}\right) .
\end{aligned}
$$

Taking the square root and using the frame indifference (4) of $W$ and Taylor expansion we get

$$
h^{-4} W\left(\nabla_{h} \hat{y}^{(h)}\right)=h^{-4} W\left(\left[\left(\nabla_{h} \hat{y}^{(h)}\right)^{T} \nabla_{h} \hat{y}^{(h)}\right]^{1 / 2}\right) \rightarrow \frac{1}{2} Q_{3}\left(A+x_{3} B\right),
$$


where

$$
\begin{aligned}
& A=\operatorname{sym} \nabla^{\prime} u+\frac{1}{2} \nabla^{\prime} v \otimes \nabla^{\prime} v+\frac{1}{2}\left|\nabla^{\prime} v\right|^{2} e_{3} \otimes e_{3}+\operatorname{sym} d^{(0)} \otimes e_{3}, \\
& B=-\left(\nabla^{\prime}\right)^{2} v+\operatorname{sym} d^{(1)} \otimes e_{3} .
\end{aligned}
$$

For a symmetric $2 \times 2$ matrix $A^{\prime \prime}$ let $c=\mathcal{L} A^{\prime \prime} \in \mathbb{R}^{3}$ denote the vector which realizes the minimum in the definition of $Q_{2}$, that is,

$$
Q_{2}\left(A^{\prime \prime}\right)=Q_{3}\left(A^{\prime \prime}+c \otimes e_{3}+e_{3} \otimes c\right) \text {. }
$$

Since $Q_{3}$ is positive definite on symmetric matrices, $c$ is uniquely determined and the map $\mathcal{L}$ is linear. We now take

$$
\begin{gathered}
d^{(0)}=-\frac{1}{2}\left|\nabla^{\prime} v\right|^{2} e_{3}+\mathcal{L}\left(\operatorname{sym} \nabla^{\prime} u+\nabla^{\prime} v \otimes \nabla^{\prime} v\right) \\
d^{(1)}=-2 \mathcal{L}\left(\left(\nabla^{\prime}\right)^{2} v\right) .
\end{gathered}
$$

Then the right-hand side of (103) reduces to

$$
\frac{1}{2} Q_{3}\left(A+x_{3} B\right)=\frac{1}{2} Q_{2}\left(G_{0}+x_{3} G_{1}\right)
$$

with

$$
G_{0}\left(x^{\prime}\right)=\operatorname{sym} \nabla^{\prime} u+\frac{1}{2} \nabla^{\prime} v \otimes \nabla^{\prime} v, \quad G_{1}\left(x^{\prime}\right)=-\left(\nabla^{\prime}\right)^{2} v .
$$

Now we drop the extra smoothness assumptions on $u$ and $v$ and we modify the ansatz (100) so that the boundary conditions for $y^{(h)}$ are satisfied exactly. To achieve this we choose maps $\bar{u}^{(h)} \in W^{1, \infty}\left(S ; \mathbb{R}^{2}\right), \bar{v}^{(h)} \in W^{2, \infty}(S)$ such that $\left(\bar{u}^{(h)}, \bar{v}^{(h)}\right) \in A_{\Gamma}$ and

$$
\bar{u}^{(h)} \rightarrow u \quad \text { in } W^{1,2}\left(S ; \mathbb{R}^{2}\right), \quad \bar{v}^{(h)} \rightarrow v \quad \text { in } W^{2,2}(S)
$$

with

$$
h^{\varepsilon}\left\|\bar{u}^{(h)}\right\|_{W^{1, \infty}}+h^{\varepsilon}\left\|\bar{v}^{(h)}\right\|_{W^{2, \infty}} \leqq C
$$

for some small power $\varepsilon>0$. To see that such maps exist one can, for example, apply the truncation result Proposition A.2 in [12] (which is essentially due to LIU [27] and ZIEMER [41]) to $u-\hat{u}$ and $v-\hat{v}$ and then add $\hat{u}$ and $\hat{v}$ respectively.

Next we consider approximations $d^{(j, h)} \in C_{0}^{\infty}(S)$ such that as $h \rightarrow 0$

$$
d^{(j, h)} \rightarrow d^{(j)} \quad \text { in } L^{2}(S), \quad h^{\varepsilon}\left\|d^{(j, h)}\right\|_{W^{1, \infty}} \leqq C .
$$

Now we make the same ansatz as in (100), that is,

$$
y^{(h)}\left(x^{\prime}, x_{3}\right)=\left(\begin{array}{c}
x^{\prime} \\
h x_{3}
\end{array}\right)+\left(\begin{array}{c}
h^{2} \bar{u}^{(h)} \\
h \bar{v}^{(h)}
\end{array}\right)-h^{2} x_{3}\left(\begin{array}{c}
\nabla^{\prime} \bar{v}^{(h)} \\
0
\end{array}\right)+h^{3} x_{3} d^{(0, h)}+\frac{h^{3}}{2} x_{3}^{2} d^{(1, h)},
$$


and we can compute $\left(\nabla_{h} y^{(h)}\right)^{T} \nabla_{h} y^{(h)}$ as above. Using Proposition 19 below and the definition of $d^{(0)}$ and $d^{(1)}$ we get

$$
h^{-4} W\left(\nabla_{h} y^{(h)}\right) \rightarrow \frac{1}{2} Q_{2}\left(G_{0}\left(x^{\prime}\right)+x_{3} G_{1}\left(x^{\prime}\right)\right) \text { in } L^{1}(\Omega) .
$$

where $G_{0}$ and $G_{1}$ are as above. This yields (97) and finishes the proof of Theorem 1.

In the argument above we have used the following convergence result.

Proposition 19. Suppose that

$$
G^{(h)} \rightarrow G \text { in } L^{2}(\Omega), \quad h^{2} G^{(h)} \rightarrow 0 \text { in } L^{\infty} .
$$

Then

$$
h^{-4} W\left(\left(I d+2 h^{2} G^{(h)}\right)^{1 / 2}\right) \rightarrow \frac{1}{2} Q_{3}(G) \text { in } L^{1}(\Omega)
$$

Proof. For a subsequence we have $G^{(h)} \rightarrow 0$ almost everywhere. Hence, for this subsequence,

$$
h^{-4} W\left(\left(I d+2 h^{2} G^{(h)}\right)^{1 / 2}\right) \rightarrow \frac{1}{2} Q_{3}(G) \text { a.e. }
$$

In view of the $L^{\infty}$ convergence we also have

$$
h^{-4} W\left(\left(I d+2 h^{2} G^{(h)}\right)^{1 / 2}\right) \leqq C h^{-4}\left|h^{2} G^{(h)}\right|^{2} \leqq\left|G^{(h)}\right|^{2} .
$$

Since the right-hand side converges in $L^{1}(\Omega)$ the generalized dominated convergence theorem implies that (77) holds along the subsequence considered. Since the limit is unique we have convergence of the full sequence.

\subsection{Normal and in-plane forces; stability alternative}

Proof of Theorem 4. As in the proof of Theorem 1 we can use the test function

$$
\check{y}\left(x^{\prime}, x_{3}\right)=\left(\begin{array}{c}
x^{\prime} \\
h x_{3}
\end{array}\right)+\left(\begin{array}{c}
h^{2} \hat{u}\left(x^{\prime}\right) \\
h \hat{v}\left(x^{\prime}\right)
\end{array}\right)-x_{3}\left(\begin{array}{c}
h^{2} \nabla^{\prime} \hat{v} \\
0
\end{array}\right)
$$

to deduce that inf ${ }_{\mathcal{A}_{\Gamma}^{h}} \leqq C h^{4}$. Thus for a minimizing sequence $y^{(h)}$ we have $J^{h}\left(y^{(h)}\right) \leqq C h^{4}$. Let

$$
e^{h}=I^{h}\left(y^{(h)}\right) .
$$

Using the estimate $W(F) \geqq c|F|^{2}-C$ with $c>0$ and Poincaré's inequality deduce first that $\left\|\nabla_{h} y^{(h)}\right\|_{L^{2}} \leqq C$ and this yields the estimate $e^{h} \leqq C h^{2}$.

Case 1. Suppose that $\lim _{\sup _{h \rightarrow 0}} h^{-4} e^{h}<\infty$. In this case the argument is completely analogous to the proof of Theorem 1 . 
Case 2. Suppose that $\limsup _{h \rightarrow 0} h^{-2} e^{h}>0$. There exists a subsequence (not relabeled) such that

$$
\lim _{h \rightarrow 0} h^{-2} I^{h}\left(y^{(h)}\right)=\lim _{h \rightarrow 0} h^{-2} e^{h}=e>0 .
$$

It follows from estimates (33) and (34) in Theorem 9 that

$$
y^{(h)} \rightarrow \bar{y} \text { in } W^{1,2}\left(\Omega ; \mathbb{R}^{3}\right), \quad \nabla_{h} y^{(h)} \rightarrow R \text { in } L^{2}\left(\Omega ; \mathbb{R}^{3 \times 3}\right)
$$

and $\bar{y} \in W^{2,2}$, while $R \in W^{1,2}, \bar{y}$ and $R$ are independent of $x_{3}$. Since $W$ vanishes only on $S O$ (3) by (6) we see that $R$ takes values in $S O(3)$ and hence $\bar{y}$ is an isometric immersion. Moreover, by part (i) of Theorem 6.1 in [12]

$$
e \geqq I^{\mathrm{Ki}}(\bar{y}) \text {. }
$$

We claim that $\bar{y} \in \mathcal{A}_{\Gamma}^{\text {iso }}$, that is, $\bar{y}$ and $\nabla^{\prime} \bar{y}$ satisfy the right boundary conditions. From (117) it follows that $y^{(h)}$ converges in $L^{2}(\Gamma)$ and together with the boundary conditions (18) for $y^{(h)}$ we see that $\bar{y}\left(x^{\prime}\right)=\left(x^{\prime}, 0\right)$ on $\Gamma$. To derive the boundary condition for $\nabla^{\prime} \bar{y}$ we consider the first moment

$$
\zeta^{(h)}=\int_{I} x_{3}\left[y^{(h)}\left(x^{\prime}, x_{3}\right)-\left(\begin{array}{c}
x^{\prime} \\
x_{3}
\end{array}\right)\right] \mathrm{d} x_{3} .
$$

By Remark 12

$$
\frac{1}{h} \zeta^{(h)} \rightarrow \frac{1}{12}(R-I d) e_{3} \text { in } W^{1,2}\left(S ; \mathbb{R}^{3}\right)
$$

Thus $h^{-1} \zeta^{(h)}$ converges in $L^{2}(\Gamma)$. Again using (18) we see that $\left|\zeta^{(h)}\right| \leqq C h^{2}$ on $\Gamma$. Hence

$$
(R-I d) e_{3}=0 \quad \mathcal{H}^{1} \text { a.e. on } \Gamma
$$

Thus

$$
R=\left(\begin{array}{rr}
R^{\prime} & 0 \\
0 & 1
\end{array}\right) \text { and } R^{\prime}=\nabla^{\prime} \bar{y}^{\prime} \in S O(2) \mathcal{H}^{1} \text { a.e. on } \Gamma
$$

Let $\tau$ be a tangent vector to $\Gamma$ (which exists $\mathcal{H}^{1}$ almost everywhere on $\Gamma$ ). Since $\bar{y}^{\prime}=i d$ on $\Gamma$ we have

$$
\nabla^{\prime} \bar{y}^{\prime} \tau=\tau \quad \mathcal{H}^{1} \text { a.e. on } \Gamma .
$$

Hence $\nabla^{\prime} \bar{y}^{\prime}=i d$ for $\mathcal{H}^{1}$ almost everywhere point in $\Gamma$ and this proves that $\bar{y} \in \mathcal{A}_{\Gamma}^{\text {iso }}$.

From (117), (118) and the hypothesis (i) we deduce that

$$
\liminf _{h \rightarrow 0} h^{-2} J^{h}\left(y^{(h)}\right)=e-\int_{\partial S} g \cdot\left(\bar{y}^{\prime}-x^{\prime}\right) \geqq J^{\mathrm{Ki}}(\bar{y}) \geqq 0 .
$$

To bound the left-hand side from above we use again the test function

$$
\check{y}^{(h)}\left(x^{\prime}, x_{3}\right)=\left(\begin{array}{c}
x^{\prime} \\
h x_{3}
\end{array}\right)+\left(\begin{array}{c}
h^{2} \hat{u}\left(x^{\prime}\right) \\
h \hat{v}\left(x^{\prime}\right)
\end{array}\right)-x_{3}\left(\begin{array}{c}
h^{2} \nabla^{\prime} \hat{v} \\
0
\end{array}\right),
$$


which belongs to $\mathcal{A}_{\Gamma}^{h}$ and satisfies $W\left(\nabla_{h} \check{y}^{(h)}\right) \leqq C h^{4}$. Hence the almost minimizing property of $y^{(h)}$ implies that

$$
0 \geqq \limsup _{h \rightarrow 0} h^{-2} J^{h}\left(y^{(h)}\right) .
$$

Thus all the inequalities in (121) and (122) must be equalities and in particular $J^{\mathrm{Ki}}(\bar{y})=0$ and

$$
I^{\mathrm{Ki}}(\bar{y})=e>0 .
$$

Hence $\bar{y}\left(x^{\prime}\right) \neq\left(x^{\prime}, 0\right)$ and this contradicts hypothesis (i).

Case 3. Suppose that $\limsup _{h \rightarrow 0} h^{-2} e^{h}=0$ and $\limsup _{h \rightarrow 0} h^{-4} e^{h}=\infty$. The argument is very similar to that given in Case 2 . Set

$$
E^{h}=e^{h}
$$

and apply Lemma 13. This yields

$$
\begin{gathered}
u^{(h)}:=\frac{h^{2}}{E^{h}} U^{(h)} \rightarrow u, \quad \text { in } W^{1,2}\left(S ; \mathbb{R}^{2}\right), \quad v^{(h)}:=\frac{h}{\sqrt{E^{h}}} V^{(h)} \rightarrow v \quad \text { in } W^{1,2}(S), \\
\frac{1}{\sqrt{E^{h}}} \zeta^{(h)} \rightarrow \frac{1}{12} A e_{3}=-\frac{1}{12}\left(\begin{array}{c}
\nabla^{\prime} v \\
0
\end{array}\right) \text { in } W^{1,2}\left(S ; \mathbb{R}^{3}\right) .
\end{gathered}
$$

In particular $u^{(h)}, v^{(h)}$ and $\zeta^{(h)}$ converge in $L^{2}(\Gamma)$ and in view of the boundary conditions (18) and the assumption on $E^{h}$ we deduce that $u=v=\nabla^{\prime} v=0$ on $\Gamma$. Now Lemma 16 implies that $2 \operatorname{sym} \nabla^{\prime} u+\nabla^{\prime} v \otimes \nabla^{\prime} v=0$ and thus $(u, v) \in \mathcal{A}_{\Gamma}^{\text {iso,lin }}$. Corollary 17 yields

$$
1=\liminf _{h \rightarrow 0} \frac{1}{E^{h}} I^{h}\left(y^{(h)}\right) \geqq I^{\mathrm{vK}}(u, v) .
$$

Moreover

$$
\frac{1}{E^{h}} \int_{\partial S} g^{(h)} \cdot\left(y^{(h)^{\prime}}-x^{\prime}\right) \mathrm{d} \mathcal{H}^{1} \rightarrow \int_{\partial S \times I} g \cdot u \mathrm{~d} \mathcal{H}^{1}
$$

and

$$
\frac{1}{E^{h}}\left|\int_{\Omega} f^{(h)} y_{3}^{(h)} \mathrm{d} x\right| \leqq \frac{h^{3}}{E^{h}} \frac{\sqrt{E^{h}}}{h}\left\|v^{(h)}\right\|_{L^{2}(S)} \rightarrow 0 .
$$

Hence

$$
\liminf _{h \rightarrow 0} \frac{1}{E^{h}} J^{h}\left(y^{(h)}\right) \geqq J^{v k}(u, v) \geqq 0,
$$

where the last inequality follows from hypothesis (ii) (and where we take $f=0$ in $J^{\mathrm{vK}}$ ). Using the same test function $\check{y}^{(h)}$ as in Case 2 we see that the left-hand side of the above inequality is bounded from above by zero. Now we conclude easily as in Case 2. 
Proof of Corollary 5. This is very similar. For the upper bound one can use the trivial map $x \mapsto\left(x^{\prime}, h x_{3}\right)$ as a test function and we get again $I^{h}\left(y^{(h)}\right) \leqq C h^{2}$. Now Case 1 can be treated as before. In Case 2 we get again $y^{(h)} \rightarrow \bar{y}$ in $W^{1,2}$, $\bar{y} \in W^{2,2}$ and $\nabla_{h} y \rightarrow R$ in $L^{2}$, with $R \in W^{1,2}$. Studying $\zeta^{(h)}$ as before we deduce that $R_{33}=1$ on $\Gamma=\{0\} \times(0,1)$. Moreover the boundary conditions in $\mathcal{B}_{\Gamma}^{h}$ imply that $\bar{y}_{1}=\bar{y}_{3}=0$ on $\Gamma$. Thus $R_{12}=\bar{y}_{1,2}=0$ on $\Gamma$. This shows that $\bar{y}_{2,2}=R_{22}= \pm 1$ on $\Gamma$. Since $\nabla^{\prime} y$ belongs to $H^{1 / 2}(\Gamma)$ it cannot have jump discontinuities. Hence $\bar{y}\left(0, x_{2}\right)=x_{2}$ or $\bar{y}\left(0, x_{2}\right)=-x_{2}$ for all $x_{2} \in(0,1)$. The second possibility is ruled out by the inequality constraint in $\mathcal{B}_{\Gamma}^{h}$. Thus we finally get $R=I d$ and $\bar{y}_{, 1}=e_{1}, \bar{y}_{, 2}=e_{2}$ on $\Gamma$. Moreover $\bar{y}_{1}=0$ and $\bar{y}_{2}=x_{2}+c_{2}$ on $\Gamma$. In view of the equality constraint in $\mathcal{B}_{\Gamma}^{h}$ we get $c_{2}=0$. Thus $y \in \mathcal{A}_{\Gamma}^{\text {iso }}$. Now the argument in Case 2 proceeds as before.

In Case 3 we use Corollary 14. The possibility of a 180 degree in-plane rotation in case (ii) (in our situation we have $\alpha=\frac{\pi}{2}, \theta=\pi$ ) of that Corollary is ruled out by the inequality constraint in $\mathcal{B}_{\Gamma}^{h}$. Thus the argument can be concluded as before.

\section{Nonlinear stability versus linearized stability}

In this section we show that condition (i) (nonlinear stability) and condition (ii) (stability in the geometrically linear setting) in Theorem 4 are essentially equivalent if the portion $\Gamma$ of the boundary of $S$, where the plate is clamped consists of a single interval. For brevity we refer to condition (ii) as linearized stability, even though the underlying problem is nonlinear due to the constraint $\operatorname{det}\left(\nabla^{\prime}\right)^{2} v=0$. As above we temporarily write $J_{g}^{\mathrm{Ki}}$ for the Kirchhoff functional in (26) and $J_{g}^{\mathrm{vK}}$ for the von Kármán functional in (15) (with $f=0$ ). Recall that the sets of admissible functions $\mathcal{A}_{\Gamma}^{\text {iso }}$ and $\mathcal{A}_{\Gamma}^{\text {iso,lin }}$ are defined in (27) and (28), respectively.

Theorem 20. (Nonlinear stability implies geometrically linear stability) Let $S$ be a bounded, simply connected Lipschitz domain and suppose that $\Gamma$ is a compact, nontrivial interval. Suppose further that $J_{g}^{\mathrm{Ki}}(y) \geqq 0$ for all $y \in \mathcal{A}_{\Gamma}^{\mathrm{iso}}$. Then $J_{g}^{\mathrm{vK}}(u, v) \geqq$ 0 for all $(u, v) \in \mathcal{A}_{\Gamma}^{\text {iso,lin }}$. If $\varepsilon \in(0,1)$ then $J_{(1-\varepsilon) g}^{\mathrm{vK}}>0$ on $\mathcal{A}_{\Gamma}^{\text {iso,lin }} \backslash\{(0,0)\}$.

Proof. This essentially follows from the fact that linearized isometric immersions are the tangent space of isometric immersions at the identity. Some care is needed with the regularity of the relevant maps, so we give the argument in detail.

We first show that $J_{g}^{\mathrm{vK}} \geqq 0$ on $\mathcal{A}_{\Gamma}^{\text {iso,lin }}$. Note that in view of Proposition 36 we have $\operatorname{det}\left(\nabla^{\prime}\right)^{2} v=0$. Suppose there exists $(u, v) \in \mathcal{A}_{\Gamma}^{\text {iso, lin }}$ with $J_{g}^{\mathrm{vK}}(u, v)<0$. Suppose for a moment that in addition $v \in W^{1, \infty}$. Let $\delta>0$ be a small number and apply Theorem 33 with $V=\delta v$. Thus we obtain an isometric immersion

$$
y_{\delta}: S \rightarrow \mathbb{R}^{3}, \quad y_{\delta}=\left(\begin{array}{c}
\Phi_{\delta} \\
\delta v
\end{array}\right)
$$

and we have the estimate

$$
\left\|\left(\nabla^{\prime}\right)^{2} \Phi\right\|_{L^{2}} \leqq C\left\|\nabla^{\prime} V\right\|_{L^{\infty}}\left\|\left(\nabla^{\prime}\right)^{2} V\right\|_{L^{2}} \leqq C \delta^{2} .
$$


By assumption $\nabla^{\prime} v=0$ on $\Gamma$ and in view of the explicit form of $\nabla^{\prime} \Phi$ (see (212) and (213)) and Lemma 45 we see that $\nabla^{\prime} \Phi_{\delta}$ is a constant in $S O(2)$ on $\Gamma$. Hence by applying a rigid motion we may assume that $\nabla^{\prime} \Phi_{\delta}=I d$ and $\Phi_{\delta}=i d$ on $\Gamma$. Note that application of a rigid motion does not affect the estimate (123). Using this estimate in connection with the Poincaré inequality we deduce that (for a subsequence)

$$
\frac{\Phi_{\delta}-i d}{\delta^{2}} \rightarrow \bar{u} \text { in } W^{2,2}\left(S ; \mathbb{R}^{2}\right)
$$

Together with the fact that $y_{\delta}$ is an isometric immersion this implies that $2 \mathrm{sym} \nabla^{\prime} \bar{u}+$ $\nabla^{\prime} v \otimes \nabla^{\prime} v=0$. Moreover $\bar{u}=0$ on $\Gamma$, since $\Phi_{\delta}=i d$ on $\Gamma$. Hence by the uniqueness result in Proposition 36 we have $\bar{u}=u$. Therefore

$$
\frac{1}{\delta^{2}} \int_{\partial S} g \cdot\left(y_{\delta}^{\prime}-x^{\prime}\right) \mathrm{d} \mathcal{H}^{1} \rightarrow \int_{\partial S} g \cdot u \mathrm{~d} \mathcal{H}^{1} .
$$

We finally claim that $\delta^{-1} A_{\delta} \rightarrow-\left(\nabla^{\prime}\right)^{2} v$ in $L^{2}$, where $A_{\delta}$ is the second fundamental form of $y_{\delta}$. Together with the above convergence result this will show that

$$
\frac{1}{\delta^{2}} J_{g}^{\mathrm{Ki}}\left(y_{\delta}\right) \rightarrow J_{g}^{\mathrm{vK}}(u, v)<0
$$

and this finishes the proof under the additional assumption $v \in W^{1, \infty}$. To obtain the convergence of $A_{\delta}$ note that

$$
\frac{1}{\delta}\left(A_{\delta}\right)_{\alpha \beta}=-\frac{1}{\delta}\left(y_{\delta}\right)_{, \alpha \beta} \cdot n_{\delta}
$$

Now

$$
\frac{1}{\delta}\left(y_{\delta}\right)_{, \alpha \beta} \rightarrow\left(\begin{array}{c}
0 \\
v_{, \alpha \beta}
\end{array}\right) \text { in } L^{2}\left(S ; \mathbb{R}^{3}\right)
$$

and $n_{\delta} \rightarrow e_{3}$ boundedly almost everywhere. This yields the desired convergence of $\delta^{-1} A_{\delta}$.

Now we remove the additional assumption $v \in W^{1, \infty}$. Indeed for a general pair $(u, v) \in \mathcal{A}_{\Gamma}^{\text {iso,lin }}$ there exist, in view of Theorem 43 approximations $v_{k} \in W^{2,2} \cap W^{1, \infty}$ with $v_{k} \rightarrow v$ in $W^{2,2}, \operatorname{det}\left(\nabla^{\prime}\right)^{2} v_{k}=0$ and $v_{k}=\nabla^{\prime} v_{k}=0$ on $\Gamma$. Hence by Proposition 36 there exists a unique $u_{k}$ such that $\left(u_{k}, v_{k}\right) \in \mathcal{A}_{\Gamma}^{\text {iso,lin }}$. Moreover $u_{k} \rightarrow u$ in $W^{1, p}$ for all $p<\infty$, in view of the convergence of $v_{k}$, the equation for $u_{k}$ and Korn's inequality (38). Thus $J_{g}^{\mathrm{vK}}\left(u_{k}, v_{k}\right) \rightarrow J_{g}^{v k}(u, v)$ and in particular $J_{g}^{\mathrm{vK}}\left(u_{k}, v_{k}\right)<0$ for some $k$. By the argument given above there exist $y_{k, \delta} \in \mathcal{A}_{\Gamma}^{\text {iso }}$ such that $J_{g}^{\mathrm{Ki}}\left(y_{k, \delta}\right)<0$.

Now let $\varepsilon \in(0,1)$ and suppose that there exist $(u, v) \in \mathcal{A}_{\Gamma}^{\text {iso,lin }}$ with

$$
J_{(1-\varepsilon) g}^{\mathrm{vK}}(u, v)=I^{\mathrm{vK}}(u, v)-(1-\varepsilon) \int_{\partial S} g \cdot u \mathrm{~d} \mathcal{H}^{1} \leqq 0 .
$$


If $\int_{\partial S} g \cdot u \leqq 0$ then $I^{\mathrm{vK}}(u, v) \leqq 0$ and using the boundary conditions for $v$ and $u$ we easily deduce that $u=v=0$ (see Proposition 36). If $\int_{\partial S} g \cdot u>0$ then

$$
J_{g}^{\mathrm{vK}}(u, v)=J_{(1-\varepsilon) g}^{\mathrm{vK}}(u, v)-\varepsilon \int_{\partial S} g \cdot u \mathrm{~d} \mathcal{H}^{1}<0
$$

and this contradicts what we have already shown.

Now we come to the converse implication: linearized stability implies stability.

Theorem 21. (Linearized stability implies nonlinear stability) Let $S$ be a bounded, simply connected Lipschitz domain and suppose that $\Gamma \subset \partial S$ is a closed, nontrivial interval. Suppose that the quadratic form $Q_{2}$ is isotropic, that is, $Q_{2}(A)=\alpha|A|^{2}+$ $\beta(\operatorname{tr} A)^{2}$. Suppose that $J^{\mathrm{vK}} \geqq 0$ on $\mathcal{A}_{\Gamma}^{\text {iso,lin }}$ and if $J^{\mathrm{vK}}(u, v)=0$ then $(u, v)=0$. Then $J^{\mathrm{Ki}} \geqq 0$ on $\mathcal{A}_{\Gamma}^{\text {iso }}$ and $\bar{J}^{\mathrm{Ki}}(y)=0$ for $y \in \mathcal{A}_{\Gamma}^{\text {iso }}$ only if $y\left(x^{\prime}\right)=\left(x^{\prime}, 0\right)$.

We first observe that there exists a symmetric stress field $\sigma: S \rightarrow \mathbb{R}_{\mathrm{sym}}^{2 \times 2}$ which is compatible with the boundary loads $g$ in the sense that

$$
\operatorname{div} \sigma=0 \quad \text { in } S, \quad-\sigma \nu=g \quad \text { on } \partial S \backslash \Gamma .
$$

To see this we consider maps $w: S \rightarrow \mathbb{R}^{2}$ and the functional

$$
\frac{1}{2} \int_{S}\left|\operatorname{sym} \nabla^{\prime} w\right|^{2} \mathrm{~d} x^{\prime}-\int_{\partial S} g \cdot w \mathrm{~d} \mathcal{H}^{1}
$$

In view of Korn's inequality this functional has a unique minimizer $\bar{w}$ in the class of $W^{1,2}$ maps which satisfy $w=0$ on $\Gamma$. Set $\sigma=-\operatorname{sym} \nabla^{\prime} \bar{w}$. Taking into account the symmetry of $\sigma$ we see that the Euler-Lagrange equation for $\bar{w}$ is

$$
\int_{S} \sigma: \nabla^{\prime} \varphi \mathrm{d} x^{\prime}+\int_{\partial S} g \cdot \varphi \mathrm{d} \mathcal{H}^{1}=0, \quad \text { for all } \varphi \in W^{1,2}\left(S, \mathbb{R}^{2}\right) \text { with } \varphi_{\mid \Gamma}=0 \text {. }
$$

This is exactly the weak form of (126), and it is this weak form which we will be using in the following.

Next we show that linearized stability can be characterized in terms of the out-of-plane deformation $v$ alone. Note that in view of (5), (6), (8) and (9) the quadratic form $Q_{2}$ is positive definite on symmetric matrices.

Lemma 22. Let $S, \Gamma, Q_{2}, g$ and $\sigma$ be as in Theorem 21. Consider the condensed functional

$$
J^{\text {cond }}(v):=\int_{S} \frac{1}{24} Q_{2}\left(\left(\nabla^{\prime}\right)^{2} v\right)-\frac{1}{2} \sigma: \nabla^{\prime} v \otimes \nabla^{\prime} v \mathrm{~d} x^{\prime}
$$

and the admissible set

$$
\mathcal{A}_{\Gamma}^{\text {cond }}:=\left\{v \in W^{2,2}(S): \operatorname{det}\left(\nabla^{\prime}\right)^{2} v=0, v=\nabla^{\prime} v=0 \text { on } \Gamma\right\} .
$$

Then

$$
J^{\mathrm{vK}} \geqq 0 \quad \text { on } \mathcal{A}_{\Gamma}^{\text {iso,lin }}
$$


if and only if

$$
J^{\text {cond }} \geqq 0 \text { on } \mathcal{A}_{\Gamma}^{\text {cond }} .
$$

Moreover equality holds in (130) only for $(u, v)=(0,0)$ if and only if equality holds in (131) only for $v=0$.

Proof. If $(u, v) \in \mathcal{A}_{\Gamma}^{\text {iso,lin }}$ then

$$
2 \operatorname{sym} \nabla^{\prime} u+\nabla^{\prime} v \otimes \nabla^{\prime} v=0
$$

and by Proposition 36 we have $\operatorname{det}\left(\nabla^{\prime}\right)^{2} v=0$. Hence $v \in \mathcal{A}_{\Gamma}^{\text {cond }}$. Moreover, applying (127) with $\varphi=u$ and using the symmetry of $\sigma$ we get

$$
-\int_{\partial S} g \cdot u \mathrm{~d} \mathcal{H}^{1}=\int_{S} \sigma: \nabla^{\prime} u \mathrm{~d} x^{\prime}=-\frac{1}{2} \int_{S} \sigma: \nabla^{\prime} v \otimes \nabla^{\prime} v \mathrm{~d} x^{\prime} .
$$

Hence $J^{\mathrm{vK}}(u, v)=J^{\text {cond }}(v)$.

Now suppose that $v \in \mathcal{A}_{\Gamma}^{\text {cond }}$. By Proposition 36 there exists a $u$ such that (132) holds. Moreover by the explicit formula (216) for $u$ and Lemma 45 we see that $\nabla^{\prime} u$ is constant on $\Gamma$. In view of (132) $\nabla^{\prime} u$ is also skew-symmetric on $\Gamma$. Hence after substraction of an affine function (with skew-symmetric gradient) we may assume that $u=0$ on $\Gamma$ (note that this modification does not affect the validity of (132)). Thus $(u, v) \in \mathcal{A}_{\Gamma}^{\text {iso,lin }}$ and we find again $J^{\mathrm{vK}}(u, v)=J^{\text {cond }}(v)$.

Proof of Theorem 21. First note that

$$
Q_{2}(A)=Q_{2}\left(\left(\nabla^{\prime}\right)^{2} y_{1}\right)+Q_{2}\left(\left(\nabla^{\prime}\right)^{2} y_{2}\right)+Q_{2}\left(\left(\nabla^{\prime}\right)^{2} y_{3}\right) .
$$

Indeed we have $-y_{, i j}=A_{i j} n$ by (204) and thus $|A|^{2}=\sum_{k=1}^{3}|A|^{2} n_{k}^{2}=\sum_{i, j, k} y_{k, i j}^{2}$ and $(\operatorname{tr} A)^{2}=\sum_{k}(\operatorname{tr} A)^{2} n_{k}^{2}=\sum_{k}\left(\Delta y_{k}\right)^{2}$. Now set

$$
\tilde{u}=\left(\begin{array}{l}
y_{1} \\
y_{2}
\end{array}\right)-\left(\begin{array}{l}
x_{1} \\
x_{2}
\end{array}\right), \quad v=y_{3} .
$$

Since $y \in A_{\Gamma}^{\text {iso }}$ we have

$$
-2 \operatorname{sym} \nabla^{\prime} \tilde{u}=\nabla^{\prime} \tilde{u}_{1} \otimes \nabla^{\prime} \tilde{u}_{1}+\nabla^{\prime} \tilde{u}_{2} \otimes \nabla^{\prime} \tilde{u}_{2}+\nabla^{\prime} v \otimes \nabla^{\prime} v .
$$

By (204) and (206) we have $\operatorname{det}\left(\nabla^{\prime}\right)^{2} y_{i}=0$ for $i=1,2,3$. Together with the boundary conditions in the definition of $\mathcal{A}_{\Gamma}^{\text {iso }}$ we see that the maps $\tilde{u}_{1}, \tilde{u}_{2}$ and $v$ all belong to $\mathcal{A}_{\Gamma}^{\text {cond }}$. Arguing as in (133) we deduce from (135)

$$
\begin{aligned}
-\int_{\partial S} g \cdot u \mathrm{~d} \mathcal{H}^{1} & =\int_{S} \sigma: \nabla^{\prime} u \mathrm{~d} x^{\prime} \\
& =-\frac{1}{2} \sum_{i=1}^{2} \int_{S} \sigma: \nabla^{\prime} \tilde{u}_{i} \otimes \nabla^{\prime} \tilde{u}_{i} \mathrm{~d} x^{\prime}-\frac{1}{2} \int_{S} \sigma: \nabla^{\prime} v \otimes \nabla^{\prime} v \mathrm{~d} x^{\prime}
\end{aligned}
$$

Together with (134) we get

$$
J^{\mathrm{Ki}}(y)=J^{\text {cond }}\left(\tilde{u}_{1}\right)+J^{\text {cond }}\left(\tilde{u}_{2}\right)+J^{\text {cond }}(v) .
$$

Thus Theorem 21 follows from Lemma 22. 


\section{An example: a rectangular plate under compression}

As an example we consider the stability of a rectangular plate which is clamped on one side and subject to a compressive force $h^{2} p$ on the opposite side, with the remaining sides being traction-free. With our previous notation this corresponds to the choices

$$
\begin{gathered}
S=(0, L) \times(0, l), \quad \Gamma=\{0\} \times(0, l), \\
g=-\sigma v \quad \text { on } \partial S, \quad \sigma=\left(\begin{array}{ll}
p & 0 \\
0 & 0
\end{array}\right), \quad p>0 .
\end{gathered}
$$

We assume again that the elastic moduli are isotropic, that is,

$$
\begin{gathered}
Q_{3}(F)=\frac{\partial^{2} W}{\partial F^{2}}(I d)(F, F)=2 \mu|\operatorname{sym} F|^{2}+\lambda(\operatorname{tr} F)^{2}, \quad \text { for } F \in \mathbb{R}^{3 \times 3} \\
Q_{2}(G)=\min _{a \in \mathbb{R}^{3}} Q_{3}\left(G+a \otimes e_{3}+e_{3} \otimes a\right) \\
=\alpha|\operatorname{sym} G|^{2}+\beta(\operatorname{tr} G)^{2}, \quad \text { for } G \in \mathbb{R}^{2 \times 2}
\end{gathered}
$$

where $\alpha=2 \mu, \beta=\frac{2 \mu \lambda}{2 \mu+\lambda}$, see (10). We always assume that $Q_{2}$ is positive definite on symmetric matrices, that is,

$$
\alpha>0, \quad \alpha+2 \beta>0 .
$$

This is equivalent to the usual conditions $\mu>0,2 \mu+3 \lambda>0$. As before we set

$$
I=(-1 / 2,1 / 2), \quad \Omega=S \times I .
$$

The three-dimensional problem consists in minimizing

$$
\begin{aligned}
J^{h}(y) & =\int_{\Omega} W\left(\nabla_{h} y\right) \mathrm{d} x-\int_{\partial S \times I} h^{2} g \cdot\left(y^{\prime}-x^{\prime}\right) \mathrm{d} \mathcal{H}^{2} \\
& =\int_{\Omega} W\left(\nabla_{h} y\right) \mathrm{d} x+\int_{x_{1}=L} h^{2} p\left(y_{1}-x_{1}\right) \mathrm{d} x_{2} \mathrm{~d} x_{3} \\
& =\int_{\Omega} W\left(\nabla_{h} y\right)+h^{2} p\left(y_{1,1}-1\right) \mathrm{d} x
\end{aligned}
$$

in the admissible set

$$
\begin{gathered}
\mathcal{B}_{\Gamma}^{h}=\left\{y^{(h)} \in W^{1,2}\left(\Omega ; \mathbb{R}^{3}\right): y_{1}^{(h)}(x)=0, y_{3}^{(h)}(x)=h x_{3} \text { on } \Gamma,\right. \\
\left.\int_{\Gamma} x_{2} y_{2}^{(h)}(x) \mathrm{d} x_{2} \geqq 0, \int_{\Gamma} y_{2}^{(h)} \mathrm{d} x_{2}=0\right\} .
\end{gathered}
$$

We do not prescribe a hard boundary condition for $y_{2}^{(h)}$. Thus the plate can expand in $x_{2}$ direction in response to a compressive stress in $x_{1}$ direction. The two additional constraints rule out degenerate behaviour under the dead loading we consider. The inequality condition rules a 180 degree in-plane rotation, which would convert the compressive load into a tensile one The equality constraint simply removes the freedom of a translation in the $x_{2}$ direction (which would not effect the energy). 


\subsection{Euler-Bernoulli theory}

We first investigate the functional $J^{h}$ on a rather restrictive class of deformations of Euler-Bernoulli type. These depend only trivially on $x_{2}$ and correspond to a bending of the strip $(0, L) \times I$ in the $\left(x_{1}, x_{3}\right)$ plane with the midline $x_{3}=0$ being mapped isometrically. More precisely we consider deformations of the form

$$
y\left(x_{1}, x_{2}, x_{3}\right)=\left(\begin{array}{c}
0 \\
x_{2} \\
0
\end{array}\right)+\int_{0}^{x_{1}}\left(\begin{array}{c}
\cos \theta(s) \\
0 \\
\sin \theta(s)
\end{array}\right) \mathrm{d} s+h x_{3} b\left(x_{1}\right)+\frac{1}{2} h^{2} x_{3}^{2} \mathrm{~d}\left(x_{1}\right),
$$

where

$$
b\left(x_{1}\right)=\left(\begin{array}{c}
-\sin \theta\left(x_{1}\right) \\
0 \\
\cos \theta\left(x_{1}\right)
\end{array}\right)
$$

Then

$$
\nabla_{h} y=R_{\theta}\left[I d-h x_{3} \theta^{\prime} e_{1} \otimes e_{1}+h x_{3} R_{\theta}^{T} \mathrm{~d} \otimes e_{3}\right]+\mathcal{O}\left(h^{2}\right)
$$

where

$$
R_{\theta}=\left(\begin{array}{ccc}
\cos \theta & 0 & -\sin \theta \\
0 & 1 & 0 \\
\sin \theta & 0 & \cos \theta
\end{array}\right)
$$

Taylor expansion and an optimal choice of $d$ (which affects the passage from $Q_{3}$ to $Q_{2}$ ) yield

$$
W\left(\nabla_{h} y\right)=\frac{h^{2} x_{3}^{2}}{2} Q_{2}\left(e_{1} \otimes e_{1}\right)\left(\theta^{\prime}\right)^{2}+\mathcal{O}\left(h^{3}\right) .
$$

Hence

$$
J^{h}(y)=h^{2} E(\theta)+\mathcal{O}\left(h^{3}\right),
$$

where $E$ is the energy functional of an Euler beam subject to a load $p$, given by

$$
E(\theta)=\int_{0}^{L} \frac{1}{24}(\alpha+\beta)\left(\theta^{\prime}\right)^{2}+p(\cos \theta-1) .
$$

The boundary condition on $y$ holds if an only if

$$
\theta(0)=0 .
$$

Since $\cos \theta-1 \geqq-\frac{1}{2} \theta^{2}$ the functional $E$ can be bounded from below by a quadratic functional for which the critical value of $p$ can be easily determined. Set

$$
p_{\mathrm{cr}}=\frac{1}{12} \frac{\pi^{2}}{4 L^{2}}(\alpha+\beta)
$$


Then

(i) For $p \leqq p_{\text {cr }}$ we have $E(\theta) \geqq 0$, and $E(\theta)=0$ implies $\theta=0$.

(ii) For $p>p_{\text {cr }}$ we have $\min E<0$.

For $p>p_{\mathrm{cr}}$ and close to $p_{\mathrm{cr}}$ the minimizer of $E$ is approximately given by the eigenfunction of the linearized problem, that is,

$$
\theta=A \sin \left(\pi x_{1} / 2 L\right)
$$

with $A \sim \sqrt{\left(p-p_{\mathrm{cr}}\right) / p}$.

Thus for $p>p_{\text {cr }}$ we have inf $J^{h} \leqq-C h^{2}$ and we are in the Kirchhoff scaling regime corresponding to a nonlinear instability. For $p<p_{\text {cr }}$ we have stability in the restricted class of Euler-Bernoulli deformation. We now investigate whether this still holds in the general class of deformations.

\subsection{Geometrically linear stability}

Here we study the functional

$$
J^{\mathrm{vK}}(u, v)=\frac{1}{24} \int_{S} Q_{2}\left(\left(\nabla^{\prime}\right)^{2} v\right) \mathrm{d} x^{\prime}+\int_{0}^{l} p u_{1}\left(L, x_{2}\right) \mathrm{d} x_{2},
$$

subject to the boundary conditions

$$
u=v=\partial_{1} v=0 \quad \text { on } \Gamma=\{0\} \times(0, l)
$$

and the linearized isometry constraint

$$
2 \operatorname{sym} \nabla^{\prime} u+\nabla^{\prime} v \otimes \nabla^{\prime} v=0 \text { in } S .
$$

We are interested in the largest value of $p$ for which $J^{\mathrm{vK}}$ is still non-negative.

Using (150) and (151) we can rewrite the loading term in terms of $v$

$$
\int_{0}^{l} u_{1}\left(L, x_{2}\right) \mathrm{d} x_{2}=\int_{S} u_{1,1} \mathrm{~d} x^{\prime}=-\frac{1}{2} \int_{S} v_{, 1}^{2} \mathrm{~d} x^{\prime} .
$$

Thus

$$
J^{\mathrm{vK}}(u, v)=Q(v):=\int_{S} \frac{1}{24} Q_{2}\left(\left(\nabla^{\prime}\right)^{2} v\right)-\frac{1}{2} p v_{, 1}^{2} \mathrm{~d} x^{\prime}
$$

Moreover (151) implies that

$$
\operatorname{det}\left(\nabla^{\prime}\right)^{2} v=0
$$

(see Proposition 36). For symmetric $2 \times 2$ matrices we have the relation

$$
(\operatorname{tr} A)^{2}=|A|^{2}+2 \operatorname{det} A
$$

Hence we have the further simplification

$$
J^{\mathrm{vK}}(u, v)=Q(v)=F(v):=\int_{S} \frac{\alpha+\beta}{24}\left|\left(\nabla^{\prime}\right)^{2} v\right|^{2}-\frac{1}{2} p v_{, 1}^{2} \mathrm{~d} x^{\prime}
$$


and

$$
F(v) \geqq G(v):=\int_{0}^{l} \int_{0}^{L} \frac{\alpha+\beta}{24}\left|v_{, 11}\right|^{2}-\frac{1}{2} p v_{, 1}^{2} \mathrm{~d} x_{1} \mathrm{~d} x_{2} .
$$

The inner integral is exactly the linearization of the Euler-Bernoulli functional discussed above (with the identification $\theta=v, 1$ ). Thus the inner integral, and hence $J^{\mathrm{vK}}$ is positive definite if $p<p_{\mathrm{cr}}$. Hence linearized stability holds for $p<p_{\mathrm{cr}}$. Tracing back the inequalities we see at $p=p_{\text {cr }}$ the nontrivial solution is a multiple of

$$
\bar{v}(x)=\cos \left(\frac{\pi x_{1}}{2 L}\right)-1
$$

and in particular is independent of $x_{2}$.

\subsection{Nonlinear stability}

The assumptions of Theorem 21 are satisfied. Hence nonlinear stability holds for $p<p_{\mathrm{cr}}$. It follows from the discussion of the Euler-Bernoulli deformations that nonlinear stability cannot hold for $p>p_{\mathrm{cr}}$. Alternatively one can plug in test functions of the form $y\left(x_{1}, x_{2}\right)=x_{2} e_{2}+\int_{0}^{x_{1}} \cos \theta e_{1}+\int_{0}^{x_{1}} \sin \theta e_{3}$ into $J^{\mathrm{Ki}}$ and recover $E(\theta)$.

\subsection{First summary}

For

$$
p<p_{\mathrm{cr}}=\frac{1}{12} \frac{\pi^{2}}{4 L^{2}}(\alpha+\beta)=\frac{\pi^{2}}{24 L^{2}} \mu \frac{2 \mu+2 \lambda}{2 \mu+\lambda} .
$$

conditions (i) and (ii) of Theorem 4 are satisfied. Hence in view of Corollary 5 we are in the vK scaling regime, the in-plane displacements are of order $h^{2}$, the out-of-plane displacement is of order $h$, the rescaled displacements satisfy $\left(u^{(h)}, v^{(h)}\right) \rightarrow(u, v)$ in $W^{1,2}$ and $(u, v)$ minimizers $J^{\mathrm{vK}}$ in $\mathcal{A}_{\Gamma}$.

If

$$
p>p_{\mathrm{cr}}
$$

then inf $J^{h} \leqq-C h^{2}$ and we are in the Kirchhoff scaling regime, with both in-plane and out-of-plane deformation being of order 1 .

\subsection{Behaviour below $p_{\mathrm{cr}}, v K$ buckling}

We now investigate in more detail the behaviour for $0<p<p_{\mathrm{cr}}$. In this regime vK theory applies, the in-plane and out-of-plane displacements are of order 
$h^{2}$ and $h$, respectively, and their limiting behaviour is governed by minimizers of the functional

$$
\begin{aligned}
J^{\mathrm{vK}}(u, v)= & \frac{1}{2} \int_{S} Q_{2}\left(\operatorname{sym} \nabla^{\prime} u+\frac{\nabla^{\prime} v \otimes \nabla^{\prime} v}{2}\right) \mathrm{d} x^{\prime} \\
& +\frac{1}{24} \int_{S} Q_{2}\left(\left(\nabla^{\prime}\right)^{2} v\right) \mathrm{d} x^{\prime}+\int_{S} p u_{1,1} \mathrm{~d} x^{\prime} .
\end{aligned}
$$

By Corollary 5 the admissible pairs $(u, v)$ are given by

$$
\mathcal{B}_{\Gamma}:=\left\{(u, v) \in W^{1,2}\left(S, \mathbb{R}^{2}\right) \times W^{2,2}(S): u_{1}=v=\nabla^{\prime} v=0 \text { on } \Gamma, \int_{\Gamma} u_{2} \mathrm{~d} x_{2}=0\right\} .
$$

The Euler-Lagrange equations for $J^{\mathrm{vK}}$ always admit a (trivial) solution of the form $(\bar{u}, 0)$. We ask for which values of $p$ this solution is globally minimizing. The first result is contained in Proposition 23 and states that the trivial solution is minimizing exactly for $0 \leqq p \leqq q_{\mathrm{cr}}$, with $q_{\mathrm{cr}}<p_{\mathrm{cr}}$ (if $\beta \neq 0$ ), that is, there is a bifurcation in the vK equations before the (global) bifurcation at $p_{\mathrm{cr}}$ discussed above.

To derive this assertion note first that $\bar{u}$ is determined by a linear elasticity equation. In fact, for the boundary conditions (153) the displacement $\bar{u}$ can be found by pointwise minimization of the integrand and we obtain

$$
\bar{u}(x)=F x,
$$

where $F$ satisfies

$$
\alpha F+\beta(\operatorname{tr} F) I d=-\left(\begin{array}{ll}
p & 0 \\
0 & 0
\end{array}\right) .
$$

Set $u=\bar{u}+\tilde{u}$. Then

$$
\begin{aligned}
& \frac{1}{2} Q_{2}\left(\operatorname{sym} \nabla^{\prime} u+\frac{\nabla^{\prime} v \otimes \nabla^{\prime} v}{2}\right)+p u_{1,1} \\
& =\frac{1}{2} Q_{2}\left(\operatorname{sym} \nabla^{\prime} \tilde{u}+\frac{\nabla^{\prime} v \otimes \nabla^{\prime} v}{2}\right)+B_{2}\left(\operatorname{sym} \nabla^{\prime} \tilde{u}+\frac{\nabla^{\prime} v \otimes \nabla^{\prime} v}{2}, F\right) \\
& \quad+\frac{1}{2} Q_{2}(F)+p F_{11}+p \tilde{u}_{1,1},
\end{aligned}
$$

where $B_{2}$ is the symmetric bilinear form associated to $Q_{2}$, that is,

$$
B_{2}(A, B)=\alpha A: B+\beta(\operatorname{tr} A)(\operatorname{tr} B)=A:(\alpha B+\beta(\operatorname{tr} B) I d) .
$$

Hence by (154) we have

$$
B_{2}\left(\operatorname{sym} \nabla^{\prime} \tilde{u}+\frac{\nabla^{\prime} v \otimes \nabla^{\prime} v}{2}, F\right)=-p\left(\tilde{u}_{1,1}+\frac{v_{, 1}^{2}}{2}\right) .
$$


Setting $C=\frac{1}{2} Q_{2}(F)+p F_{11}$ we can thus write the right-hand side of (155) as

$$
\frac{1}{2} Q_{2}\left(\operatorname{sym} \nabla^{\prime} \tilde{u}+\frac{\nabla^{\prime} v \otimes \nabla^{\prime} v}{2}\right)-p v_{, 1}^{2}+C .
$$

Thus we can eliminate $\bar{u}$ and consider the new functional

$$
\begin{aligned}
\tilde{J}^{\mathrm{vK}}(\tilde{u}, v):= & J^{\mathrm{vK}}(\bar{u}+\tilde{u}, v)-|S| C \\
= & \frac{1}{2} \int_{S} Q_{2}\left(\operatorname{sym} \nabla^{\prime} \tilde{u}+\frac{\nabla^{\prime} v \otimes \nabla^{\prime} v}{2}\right) \mathrm{d} x^{\prime} \\
& \times \int_{S} \frac{1}{24} Q_{2}\left(\left(\nabla^{\prime}\right)^{2} v\right)-\frac{1}{2} p v_{, 1}^{2} \mathrm{~d} x^{\prime} .
\end{aligned}
$$

The corresponding Euler-Lagrange equation always has the trivial solution $\tilde{u}=0$, $v=0$ and we ask for which $p$ this solution is a global minimizer, i.e for which $p$ we have inf $\tilde{J}^{\mathrm{vK}} \geqq 0$. To decide this it suffices to consider the quadratic functional

$$
Q(v)=Q_{p}(v):=\int_{S} \frac{1}{24} Q_{2}\left(\left(\nabla^{\prime}\right)^{2} v\right)-\frac{1}{2} p v_{, 1}^{2} \mathrm{~d} x^{\prime} .
$$

Indeed, clearly $\tilde{J}^{\mathrm{vK}} \geqq Q$. On the other hand if $Q(\bar{v})<0$ then $J^{\mathrm{vK}}(0, \varepsilon \bar{v})<0$ for sufficiently small $\varepsilon$, since then the quadratic functional $Q$ dominates the quadratic first term. Now $Q$ is exactly the functional already studied in Subsection 7.2. The difference is that we no longer impose the constraint $\operatorname{det}\left(\nabla^{\prime}\right)^{2} v=0$.

Proposition 23. There exist $q_{\mathrm{cr}} \in\left(0, p_{\mathrm{cr}}\right]$ such that

$$
\begin{aligned}
Q(v) \geqq 0 & \text { for } p \leqq q_{\mathrm{cr}}, \\
\text { inf } Q=-\infty & \text { for } p>q_{\mathrm{cr}} .
\end{aligned}
$$

For $\beta \neq 0$ we have

$$
q_{\mathrm{cr}}<p_{\mathrm{cr}}
$$

Moreover, in this case, any nontrivial function $v$ which achieves $Q(v)=0$ for $p=q_{\mathrm{cr}}$ cannot be a function of $x_{1}$ alone.

Proof. The existence of such a $q_{\mathrm{cr}}>0$ follows from the Poincaré inequality and a simple continuity argument. Clearly $q_{\mathrm{cr}} \leqq p_{\mathrm{cr}}$. Now suppose that $\beta \neq 0$ and that $q_{\mathrm{cr}}=p_{\mathrm{cr}}$. Take $p=q_{\mathrm{cr}}$ We know that the one-dimensional function

$$
\bar{v}=\cos \left(\frac{\pi x_{1}}{2 L}\right)-1
$$

achieves $Q(\bar{v})=0$. Thus it in particular minimizes $Q$. We will conclude by showing that a function of $x_{1}$ alone cannot satisfy the natural boundary conditions for $Q$ and hence cannot be a minimizer. Indeed, the minimizing property of $\bar{v}$ yields

$$
\int_{S} \frac{1}{12} \alpha\left(\left(\nabla^{\prime}\right)^{2} \bar{v}:\left(\nabla^{\prime}\right)^{2} \varphi\right)+\frac{1}{12} \beta\left(\operatorname{tr}\left(\nabla^{\prime}\right)^{2} \bar{v}\right)\left(\operatorname{tr}\left(\nabla^{\prime}\right)^{2} \varphi\right)-p_{\mathrm{cr}} \bar{v}_{, 1} \varphi, 1 \mathrm{~d} x^{\prime}=0
$$


for all $\varphi$ with $\varphi=\varphi, 1=0$ at $x_{1}=0$. Take $\varphi$ of the form $\psi\left(x_{1}\right) \eta\left(x_{2}\right)$. Then

$$
\int_{S} \frac{1}{12} \alpha \bar{v}_{, 11} \psi^{\prime \prime} \eta+\frac{1}{12} \beta \bar{v}_{, 11}\left(\psi^{\prime \prime} \eta+\psi \eta^{\prime \prime}\right)-p_{\mathrm{cr}} \bar{v}_{, 1} \psi_{, 1} \eta \mathrm{d} x^{\prime}=0
$$

Taking $\eta=1$ we find the weak form of the equation for $\bar{v}=\bar{v}\left(x_{1}\right)$. Substituting this back into (158) we get

$$
\begin{aligned}
0 & =\int_{S} \frac{1}{12} \beta \bar{v}_{, 11}\left(x_{1}\right) \psi\left(x_{1}\right) \eta^{\prime \prime}\left(x_{2}\right) \mathrm{d} x_{1} \mathrm{~d} x_{2} \\
& =\left(\int_{0}^{L} \frac{1}{12} \beta \bar{v}_{, 11} \psi\left(x_{1}\right) \mathrm{d} x_{1}\right)\left(\eta^{\prime}(l)-\eta^{\prime}(0)\right) .
\end{aligned}
$$

Since $\eta$ is arbitrary, $\beta>0$ and $\psi$ is arbitrary (subject only to $\psi(0)=\psi^{\prime}(0)=0$ ) we conclude that $\bar{v}_{, 11} \equiv 0$, which together with the boundary condition yields $\bar{v} \equiv 0$. This contradiction implies that we must have $q_{\mathrm{cr}}<p_{\mathrm{cr}}$. The same argument shows that a nontrivial minimizer for $p=q_{\text {cr }}$ cannot be a function of $x_{1}$ only.

\subsection{Behaviour of $J^{\mathrm{vK}}$ near $p_{\mathrm{cr}}$}

We now investigate in more detail the behaviour of the vK functional for $p<p_{\mathrm{cr}}$ and $p$ close to $p_{\mathrm{cr}}$. We sometimes write the functional as $J_{p}^{\mathrm{vK}}$ to emphasize the dependence on $p$. We will show that (for $\beta \neq 0$ ) the infimum of $J^{\mathrm{vK}}$ converges to $-\infty$ as $p$ approaches $p_{\text {cr }}$ from below. To do so we will use a test function which differs from the one-dimensional ansatz (which leads to the bifurcation at $p_{\mathrm{cr}}$ ) by a two-dimensional perturbation which is concentrated in a boundary layer near $x_{2}=0$ (we could also include a symmetric boundary layer at $x_{2}=1$, but the calculation is slightly shorter for a single boundary layer). Optimizing parameters we obtain an upper bound for inf $J^{\mathrm{vK}}$ which is proportional to $-\left(p-p_{\mathrm{cr}}\right)^{3}$. Using an interpolation inequality we show that there is a lower bound with the same exponent. We also identify the scaling of the minimizers $v_{p}$ as $p \rightarrow p_{\mathrm{cr}}$. The rest of this subsection may be skipped at first reading.

Theorem 24. Suppose that $\beta \neq 0$. Then

(i) inf $J_{p}^{\mathrm{vK}}$ is attained for all $p<p_{\mathrm{cr}}$.

(ii) We have $\lim _{p \rightarrow p_{\mathrm{cr}}, p<p_{\mathrm{cr}}}$ inf $J_{p}^{\mathrm{vK}}=-\infty$. More precisely, there exist constants $c>0, C>0$ such that

$$
-C\left(p_{\mathrm{cr}}-p\right)^{-3} \leqq \min J_{p}^{\mathrm{vK}} \leqq-c\left(p_{\mathrm{cr}}-p\right)^{-3}+C .
$$

(iii) If $\left(u_{p}, v_{p}\right)$ is a minimizer $J_{p}^{\mathrm{vK}}$ then, for $p<p_{\mathrm{cr}}$ sufficiently close to $p$

$$
c\left(p_{\mathrm{cr}}-p\right)^{-2} \leqq\left\|v_{p}\right\|_{L^{2}} \leqq\left\|v_{p}\right\|_{W^{2,2}} \leqq C\left(p_{\mathrm{cr}}-p\right)^{-2} .
$$

Moreover if $w_{p}$ denotes the projection of $v_{p}$ onto the subspace perpendicular to the one-dimensional minimizer $\bar{v}$ given by (152) then

$$
c\left(p_{\mathrm{cr}}-p\right)^{-3 / 2} \leqq\left\|w_{p}\right\|_{W^{2,2}} \leqq C\left(p_{\mathrm{cr}}-p\right)^{-3 / 2} .
$$


Proof. The first assertion follows from a general relation between linearized stability and attainment for $J^{\mathrm{vK}}$ (see Theorem 27 below). The main point is to establish (159). For this it suffices to consider the reduced functional $\tilde{J}^{\mathrm{vK}}$ defined in (156). From the argument for this the estimates (160) and (161) will follow easily. Step 1. Upper bound for inf $\tilde{J}_{p}^{\mathrm{vK}}$.

Recall from (156) that

$$
\tilde{J}^{\mathrm{vK}}(\tilde{u}, v)=\frac{1}{2} \int_{S} Q_{2}\left(\operatorname{sym} \nabla^{\prime} \tilde{u}+\frac{\nabla^{\prime} v \otimes \nabla^{\prime} v}{2}\right) \mathrm{d} x^{\prime}+\int_{S} \frac{1}{24} Q_{2}\left(\left(\nabla^{\prime}\right)^{2} v\right)-\frac{1}{2} p v_{, 1}^{2} .
$$

We first focus on the quadratic term in $v$ and write a general function $v$ (satisfying the boundary conditions $v=v_{, 1}=$ ) as

$$
v=A \bar{v}+w=\hat{v}+w, \quad \text { where } \int_{S} \bar{v} w \mathrm{~d} x^{\prime}=0
$$

and where

$$
\bar{v}\left(x^{\prime}\right)=\cos \left(\frac{\pi x_{1}}{2 L}\right)-1
$$

is the one-dimensional minimizer of $Q_{p_{\mathrm{cr}}}$, subject to the constraint $\operatorname{det}\left(\nabla^{\prime}\right)^{2} v=0$.

We first note that $\bar{v}$ and $\hat{v}$ minimize the functional

$$
\tilde{Q}_{p_{\mathrm{cr}}}(v):=\int_{S} \frac{1}{24}(\alpha+\beta)\left|\left(\nabla^{\prime}\right)^{2} v\right|^{2}-\frac{1}{2} p_{\mathrm{cr}} v_{, 1}^{2} \mathrm{~d} x^{\prime}
$$

among all $v$ with $v=v_{, 1}=0$ on $x_{1}=0$ (not just among those with $\operatorname{det}\left(\nabla^{\prime}\right)^{2} v=0$ ). To see this it suffices to bound the term involving the second derivatives from below by $v_{, 11}^{2}$. Hence $\tilde{Q}_{p_{\text {cr }}}(\hat{v}+w)=\tilde{Q}_{p_{\text {cr }}}(w)$. In view of the relation $(\operatorname{tr} A)^{2}=$ $|A|^{2}+2 \operatorname{det} A$ for symmetric $2 \times 2$ matrices we thus get for the functional $Q_{p_{\mathrm{cr}}}$, defined in (157),

$$
\begin{aligned}
Q_{p_{\mathrm{cr}}}(\hat{v}+w) & =\tilde{Q}_{p_{\mathrm{cr}}}(w)+\int_{S} \frac{\beta}{12} \operatorname{det}\left(\left(\nabla^{\prime}\right)^{2}(\hat{v}+w)\right) \mathrm{d} x^{\prime} \\
& =\tilde{Q}_{p_{\mathrm{cr}}}(w)+\frac{\beta}{12} \int_{S} \operatorname{cof}\left(\nabla^{\prime}\right)^{2} \hat{v}:\left(\nabla^{\prime}\right)^{2} w+\operatorname{det}\left(\left(\nabla^{\prime}\right)^{2} w\right) \mathrm{d} x^{\prime} \\
& =Q_{p_{\mathrm{cr}}}(w)+A \frac{\beta}{12} \int_{S} \bar{v}_{, 11} w_{, 22}
\end{aligned}
$$

To estimate the stretching energy, that is, the first term on the right-hand side of (162) we start from the expansion

$$
\nabla^{\prime}(\hat{v}+w) \otimes \nabla^{\prime}(\hat{v}+w)=\nabla^{\prime} \hat{v} \otimes \nabla^{\prime} \hat{v}+2 \operatorname{sym}\left(\nabla^{\prime} w \otimes \nabla^{\prime} \hat{v}\right)+\nabla^{\prime} w \otimes \nabla^{\prime} w
$$

We know that there exists $\hat{u}$ such that $\operatorname{sym} \nabla^{\prime} \hat{u}+\frac{1}{2} \nabla^{\prime} \hat{v} \otimes \nabla^{\prime} \hat{v}=0$ (indeed we can take $2 \hat{u}_{1}=\int_{0}^{x_{1}}-\hat{v}_{, 1}^{2}, \hat{u}_{2}=0$ ). We seek $u$ (satisfying the boundary condition 
$u_{1}=0$ at $\left.x_{1}=0\right)$ such that $\operatorname{sym} \nabla^{\prime} u+\operatorname{sym} \nabla^{\prime} w \otimes \nabla^{\prime} \hat{v}$ is small. To achieve this we now consider a special ansatz for $w$ :

$$
w\left(x^{\prime}\right)=\psi\left(x_{1}\right) \eta\left(x_{2}\right), \quad \text { with } \psi, \eta \in C^{\infty}, \quad \psi(0)=0, \quad \int_{0}^{l} \eta \mathrm{d} x_{2}=0 .
$$

This yields

$$
\operatorname{sym}\left(\nabla^{\prime} w \otimes \nabla^{\prime} \hat{v}\right)=A\left(\begin{array}{cc}
\psi \psi^{\prime} \eta \bar{v}^{\prime} & \frac{\psi \eta^{\prime} \bar{v}}{2} \\
\frac{\psi \eta^{\prime} \bar{v}}{2} & 0
\end{array}\right)
$$

Setting

$$
u_{1}=-A(\psi \bar{v})\left(x_{1}\right) \eta\left(x_{2}\right), \quad u_{2}=0
$$

we see that

$$
\begin{aligned}
& \left|\operatorname{sym} \nabla^{\prime} u+\operatorname{sym}\left(\nabla^{\prime} w \otimes \nabla^{\prime} \hat{v}\right)\right| \\
& \quad=|A|\left|-(\psi \bar{v})^{\prime}\left(x_{1}\right) \eta\left(x_{2}\right)+\left(\psi^{\prime} \bar{v}^{\prime}\right)\left(x_{1}\right) \eta\left(x_{2}\right)\right| \leqq C|A|\left|\eta\left(x_{2}\right)\right| .
\end{aligned}
$$

Here and in the following we do not indicate the dependence of the various constants on $\psi$. Thus

$$
\begin{aligned}
\int_{S} Q_{2}\left(\operatorname{sym} \nabla^{\prime}(\hat{u}+u)+\frac{\nabla^{\prime} v \otimes \nabla^{\prime} v}{2}\right) & \leqq C A^{2}\|\eta\|_{L^{2}}^{2}+C\left\|\left|\nabla^{\prime} w\right|^{2}\right\|_{L^{2}}^{2} \\
& \leqq C A^{2}\|\eta\|_{L^{2}}^{2}+C\|\eta\|_{W^{1,4}}^{4} .
\end{aligned}
$$

This and (164) yield

$$
\begin{aligned}
& \tilde{J}_{p_{\mathrm{cr}}}^{\mathrm{vK}}(\hat{u}+u, v) \leqq C A^{2}\|\eta\|_{L^{2}}^{2}+C\|\eta\|_{W^{1,4}}^{4} \\
& \quad+A \frac{\beta}{12}\left(\int_{0}^{L} \bar{v}_{, 11} \psi \mathrm{d} x_{1}\right)\left(\eta^{\prime}(l)-\eta^{\prime}(0)\right)+C\|\eta\|_{W^{2,2}}^{2}
\end{aligned}
$$

Now assume that $\eta$ is concentrated in a thin boundary layer, that is,

$$
\eta\left(x_{2}\right)=B \delta \rho\left(x_{2} / \delta\right),
$$

where $\rho \in C^{\infty}([0, \infty)), \rho_{[1, \infty]}=0, \rho^{\prime}(0)=1, \int \rho=0$ and take $\delta \leqq \min (1, l / 2)$. Then $\eta^{\prime}(0)-\eta^{\prime}(l)=\eta^{\prime}(0)=B$ and

$$
\|\eta\|_{L^{2}} \leqq C B \delta^{3 / 2}, \quad\|\eta\|_{W^{1,4}} \leqq C B \delta^{1 / 4}, \quad\|\eta\|_{W^{2,2}} \leqq C B \delta^{-1 / 2} .
$$

Set

$$
\varepsilon=\left(p_{\mathrm{cr}}-p\right), \quad c_{0}=\frac{\beta}{12} \int_{0}^{L} \bar{v}_{, 11} \psi \mathrm{d} x_{1}
$$

and choose $\psi$ such that $c_{0}>0$.

Now $\tilde{J}_{p}^{\mathrm{vK}}(\cdot, v)=\tilde{J}_{p_{\mathrm{cr}}}^{\mathrm{vK}}(\cdot, v)+\varepsilon \int_{S} v_{, 1}^{2}$, and (168) implies that

$$
\tilde{J}_{p}^{\mathrm{vK}}(\hat{u}+u, v) \leqq C A^{2} B^{2} \delta^{3}+C B^{4} \delta-c_{0} A B+C B^{2} \delta^{-1}+C \varepsilon\left(A^{2}+\delta B^{2}\right) .
$$


Let $B=c_{1} A \delta$, where $c_{1}$ is a small constant. Then $\delta B^{2} \leqq A^{2}$ and thus

$$
J_{p}^{\mathrm{vK}}(\bar{u}+u, v) \leqq C A^{4} \delta^{5}-c_{0} c_{1} A^{2} \delta+C c_{1}^{2} A^{2} \delta+C \varepsilon A^{2} .
$$

Finally take $c_{1}=c_{0} /(2 C)$ and $\delta=4 C \varepsilon /\left(c_{0} c_{1}\right)$. Then the right-hand side reduces to $C A^{4} \varepsilon^{5}-c A^{2} \varepsilon$. The choice $A^{2}=(c / 2 C) \varepsilon^{-4}$ finally yields

$$
\min \tilde{J}_{p}^{\mathrm{vK}} \leqq-c^{\prime} \varepsilon^{-3}
$$

This finishes the proof of the upper bound. Note that we implicitly used that $\varepsilon$ is small since otherwise the above choice for $\delta$ may not be compatible with the restriction $\delta \leqq \min (l / 2,1)$ we imposed in the definition of $\eta$.

Step 2. Lower bound for $\min \tilde{J}_{p}^{\mathrm{vK}}$.

To establish a lower bound we start from (163)

$$
Q_{p_{\mathrm{cr}}}(\hat{v}+w)=\tilde{Q}_{p_{\mathrm{cr}}}(w)+\frac{\beta}{12} \int_{S} \operatorname{cof}\left(\nabla^{\prime}\right)^{2} \hat{v}:\left(\nabla^{\prime}\right)^{2} w+\operatorname{det}\left(\nabla^{\prime}\right)^{2} w \mathrm{~d} x^{\prime} .
$$

To estimate the last term we use the stretching energy. Let $\hat{u}$ be a minimizer (subject to $\hat{u}_{1}=0$ at $x_{1}=0$ ) of

$$
\int_{S} Q_{2}\left(\operatorname{sym} \nabla^{\prime} u+\frac{\nabla^{\prime}(\hat{v}+w) \otimes \nabla^{\prime}(\hat{v}+w)}{2}\right) \mathrm{d} x^{\prime}
$$

and set

$$
f=\operatorname{sym} \nabla^{\prime} \hat{u}+\frac{\nabla^{\prime}(\hat{v}+w) \otimes \nabla^{\prime}(\hat{v}+w)}{2} .
$$

Using the compatibility condition $e_{11,22}+e_{22,11}-2 e_{12,12}$ for the symmetrized gradient $e=\operatorname{sym} \hat{u}$ we get

$$
\begin{aligned}
f_{11,22}+f_{22,11}-2 f_{12,12} & =-\operatorname{det}\left(\nabla^{\prime}\right)^{2}(\hat{v}+w) \\
& =-\operatorname{cof}\left(\nabla^{\prime}\right)^{2} v:\left(\nabla^{\prime}\right)^{2} w-\operatorname{det}\left(\nabla^{\prime}\right)^{2} w .
\end{aligned}
$$

The vK energy gives control of the $L^{2}$ norm of $f$ and hence the $H^{-2}$ norm of the right-hand side. The basic idea is now to use an interpolation inequality like

$$
\int_{S} g \mathrm{~d} x_{1} \leqq C\|g\|_{H^{-2}}^{1 / 4}\|g\|_{L^{2}}^{3 / 4}
$$

A priori it is not clear, however, that we do have good control of the $L^{2}$ norm of (171) (which would, at least at first glance, require control of $\|w\|_{W^{2,2}}$ ). Hence we first recall the argument leading to the interpolation estimate. This will allow us to exploit the special structure arising from the combination of the first and last term on the right-hand side of (170), see Proposition 25 below.

For $\delta>0$ we choose a cut-off functions $\varphi_{\delta} \in C_{0}^{\infty}(S)$ which satisfy

$$
\begin{aligned}
& \varphi_{\delta}(x)=1 \quad \text { if } \operatorname{dist}(x, \partial S) \geqq 2 \delta, \\
& \varphi_{\delta}(x)=0 \quad \text { if } \operatorname{dist}(x, \partial S) \leqq \delta, \quad\left|\left(\nabla^{\prime}\right)^{2} \varphi_{\delta}\right| \leqq C \delta^{-2} .
\end{aligned}
$$


Using (170) and (171) and the relation $2 \operatorname{det} A=(\operatorname{tr} A)^{2}-|A|^{2}$ for symmetric $2 \times 2$ matrices we get

$$
\begin{aligned}
& Q_{p_{\mathrm{cr}}}(\hat{v}+w) \\
& =\tilde{Q}_{p_{\mathrm{cr}}}(w)-\frac{\beta}{12} \int_{S} f_{11} \varphi_{\delta, 11}+f_{22} \varphi_{\delta, 11}-f_{12} \varphi_{\delta, 12} \mathrm{~d} x^{\prime} \\
& \quad+\frac{\beta}{12} \int_{S}\left(1-\varphi_{\delta}\right) \operatorname{cof}\left(\nabla^{\prime}\right)^{2} \hat{v}:\left(\nabla^{\prime}\right)^{2} w \mathrm{~d} x^{\prime}+\frac{\beta}{12} \int_{S}\left(1-\varphi_{\delta}\right) \operatorname{det}\left(\nabla^{\prime}\right)^{2} w \mathrm{~d} x^{\prime} \\
& \geqq I^{\delta}(w)-C|\beta|\left\|\varphi_{\delta}\right\|_{W^{2,2}}\|f\|_{L^{2}}-C|\beta|\left\|\left(1-\varphi_{\delta}\right)\left(\nabla^{\prime}\right)^{2} v\right\|_{L^{2}}\left\|\left(\nabla^{\prime}\right)^{2} w\right\|_{L^{2}} \\
& \geqq I^{\delta}(w)-C|\beta| \delta^{-3 / 2}\|f\|_{L^{2}}-C|\beta| \delta^{1 / 2}\left\|\left(\nabla^{\prime}\right)^{2} \hat{v}\right\|_{L^{\infty}}\left\|\left(\nabla^{\prime}\right)^{2} w\right\|_{L^{2}},
\end{aligned}
$$

where

$$
\begin{aligned}
I^{\delta}(w):= & \left.\int_{S} \varphi_{\delta} \frac{\alpha+\beta}{24}\left|\left(\nabla^{\prime}\right)^{2} w\right|^{2}+\left.\left(1-\varphi_{\delta}\right)\left[\frac{\alpha}{24} \mid\left(\nabla^{\prime}\right)^{2} w\right)\right|^{2}+\frac{\beta}{24}\left(\operatorname{tr}\left(\nabla^{\prime}\right)^{2} w\right)^{2}\right] \mathrm{d} x^{\prime} \\
& -\int_{S} \frac{1}{2} p_{\mathrm{cr}} w_{, 1}^{2} \mathrm{~d} x^{\prime} .
\end{aligned}
$$

As $\delta \rightarrow 0$ this functional approaches $Q_{p_{\text {cr }}}$, which is positive definite on the subspace orthogonal to $\bar{v}$. In fact we have a uniform lower bound on $I^{\delta}$ on that subspace for sufficiently small $\delta$.

Proposition 25. There exist $c>0$ and $\delta_{0}>0$ such that for all $\delta \in\left(0, \delta_{0}\right)$

$$
I^{\delta}(w) \geqq c\|w\|_{W^{2,2}}^{2}, \quad \text { whenever } \int_{S} w \bar{v} \mathrm{~d} x^{\prime}=0
$$

and $w=w_{, 1}=0$ on $x_{1}=0$.

Proof. Suppose otherwise. Since $I^{\delta}$ is quadratic in $w$ there then exist $\delta_{j} \rightarrow 0$ and $w_{j}$ such that

$$
\begin{gathered}
\left\|w_{j}\right\|_{W^{2,2}}=1, \quad w_{j} \rightarrow w \text { in } W^{2,2}, \quad \int_{S} w_{j} \bar{v} \mathrm{~d} x^{\prime}=0, \\
\lim _{j \rightarrow \infty} I^{\delta_{j}}\left(w_{j}\right) \leqq 0 .
\end{gathered}
$$

Fix $\delta>0$ and let $S_{\delta}=\{x \in S: \operatorname{dist}(x, \partial S) \geqq 2 \delta\}$. Since $Q_{2}(A) \geqq 0$ we have for $\delta_{j} \leqq \delta$

$$
I^{\delta_{j}}\left(w_{j}\right) \geqq \int_{S_{\delta}} \frac{\alpha+\beta}{24}\left|\left(\nabla^{\prime}\right)^{2} w_{j}\right|^{2} \mathrm{~d} x^{\prime}-\int_{S} p_{\mathrm{cr}} w_{j, 1}^{2} \mathrm{~d} x^{\prime} .
$$

Thus by standard lower semicontinuity results and the compact embedding of $W^{2,2}$ into $W^{1,2}$

$$
\begin{aligned}
\liminf _{j \rightarrow \infty} I^{\delta_{j}}\left(w_{j}\right) & \geqq \liminf _{j \rightarrow \infty} \int_{S_{\delta}} \frac{\alpha+\beta}{24}\left|\left(\nabla^{\prime}\right)^{2} w_{j}\right|^{2} \mathrm{~d} x^{\prime}-\int_{S} p_{\mathrm{cr}} w_{j, 1}^{2} \mathrm{~d} x^{\prime} \\
& \geqq \int_{S_{\delta}} \frac{\alpha+\beta}{24}\left|\left(\nabla^{\prime}\right)^{2} w\right|^{2} \mathrm{~d} x^{\prime}-\int_{S} p_{\mathrm{cr}} w_{, 1}^{2} \mathrm{~d} x^{\prime}
\end{aligned}
$$


Hence

$$
\int_{S_{\delta}} \frac{\alpha+\beta}{24}\left|\left(\nabla^{\prime}\right)^{2} w\right|^{2} \mathrm{~d} x^{\prime}-\int_{S} p_{\mathrm{cr}} w_{, 1}^{2} \mathrm{~d} x^{\prime} \leqq 0 .
$$

Since $\delta>0$ was arbitrary, the same estimate holds with $S_{\delta}$ replaced with $S$. Hence $w$ must be a multiple of $\bar{v}$. On the other hand $w$ is also perpendicular to $\bar{v}$ in view of (175). Thus $w=0$. By the compact Sobolev embedding we have $w_{j} \rightarrow 0$ in $W^{1,2}$. Since the quadratic forms $(\alpha+\beta)|A|^{2}$ and $\alpha|A|^{2}+\beta(\operatorname{tr} A)^{2}$ are positive definite on symmetric matrices we see that

$$
\limsup _{j \rightarrow \infty}\left\|\left(\nabla^{\prime}\right)^{2} w_{j}\right\|_{L^{2}}^{2} \leqq C \limsup _{j \rightarrow \infty} I^{\delta}\left(w_{j}\right) \leqq 0
$$

Thus $w_{j} \rightarrow 0$ in $W^{2,2}$. This contradicts the assumption that $\left\|w_{j}\right\|_{W^{2,2}}=1$ and the proof of the proposition is finished.

From Proposition 25 and (172) we deduce that

$$
\begin{aligned}
Q_{p_{\mathrm{cr}}}(\hat{v}+w) & \geqq c\|w\|_{W^{2,2}}^{2}-C \delta^{-3 / 2}\|f\|_{L^{2}}-C \delta^{1 / 2}\left\|\left(\nabla^{\prime}\right)^{2} \hat{v}\right\|_{L^{\infty}}\left\|\left(\nabla^{\prime}\right)^{2} w\right\|_{L^{2}} \\
& \geqq \frac{c}{2}\|w\|_{W^{2,2}}^{2}-\frac{C}{\kappa} \delta^{-3}-\kappa\|f\|_{L^{2}}^{2}-C \delta\left\|\left(\nabla^{\prime}\right)^{2} \hat{v}\right\|_{L^{\infty}}^{2},
\end{aligned}
$$

for all $\kappa>0$. Now choose $\kappa$ such that we have the pointwise bound $Q_{2}(A) \geqq$ $4 \kappa|A|^{2}$ for symmetric matrices $A$. Then, using again the abbreviation $\varepsilon=p_{\mathrm{cr}}-\bar{p}$,

$$
\begin{aligned}
\tilde{J}_{p}^{\mathrm{vK}}(u, \hat{v}+w) & \geqq \frac{1}{2} \int_{S} Q_{2}(f) \mathrm{d} x^{\prime}+Q_{p_{\mathrm{cr}}}(\hat{v}+w)+\varepsilon \int_{S}\left((\hat{v}+w)_{, 1}^{2} \mathrm{~d} x^{\prime}\right. \\
& \geqq 2 \kappa\|f\|_{L^{2}}^{2}+Q_{p_{\mathrm{cr}}}(\hat{v}+w)+\frac{\varepsilon}{2}\left\|\hat{v}_{, 1}\right\|_{L^{2}}^{2}-2 \varepsilon\left\|w_{, 1}\right\|_{L^{2}}^{2}
\end{aligned}
$$

Note that all norms of $\hat{v}$ are equivalent since $\hat{v}$ is a multiple of a fixed smooth function. Taking $\delta=\varepsilon / C^{\prime}$ we thus get

$$
\min \tilde{J}_{p}^{\mathrm{vK}}(u, \hat{v}+w) \geqq \kappa\|f\|_{L^{2}}^{2}+\frac{c}{4}\|w\|_{W^{2,2}}^{2}-\frac{C}{\kappa} \varepsilon^{-3}+\frac{\varepsilon}{4}\left\|\hat{v}_{, 1}\right\|^{2} .
$$

Thus

$$
\min \tilde{J}_{p}^{\mathrm{vK}} \geqq-C \varepsilon^{-3}
$$

which proves assertion (ii).

Step 3. Estimates for the minimizers of $\tilde{J}^{\mathrm{vK}}$.

From (176) and the equivalence of different norm of $\hat{v}$ we immediately deduce that a minimizer $\left(u_{p}, v_{p}\right)$ with $v_{p}=\hat{v_{p}}+w_{p}\left(\right.$ where $\left.\int_{S} w_{p} \bar{v}=0\right)$ satisfies

$$
\begin{aligned}
& \left\|w_{p}\right\|_{W^{2,2}} \leqq C \varepsilon^{-3 / 2}, \quad\left\|\hat{v}_{p}\right\|_{W^{2, \infty}} \leqq C \varepsilon^{-2} \\
& \left\|\operatorname{det}\left(\nabla^{\prime}\right)^{2} v_{p}\right\|_{H^{-2}} \leqq C\left\|f_{p}\right\|_{L^{2}} \leqq C \varepsilon^{-3 / 2}
\end{aligned}
$$


To prove the converse estimates we use (172). In connection with Proposition 25 and the upper bound for $J_{p}^{\mathrm{vK}}$ we get, for sufficiently small $\varepsilon$,

$$
\begin{aligned}
-c \varepsilon^{-3} & \geqq J_{p}^{\mathrm{vK}}\left(u_{p}, \hat{v}_{p}+w_{p}\right) \\
& \geqq-C \delta^{-3 / 2}\left\|f_{p}\right\|_{L^{2}}-C \delta^{1 / 2}\left\|\left(\nabla^{\prime}\right)^{2} \hat{v}_{p}\right\|_{L^{\infty}}\left\|\left(\nabla^{\prime}\right)^{2} w_{p}\right\|_{L^{2}} .
\end{aligned}
$$

Now if $\left\|\left(\nabla^{\prime}\right)^{2} \hat{v}_{p}\right\|_{\infty}\left\|\left(\nabla^{\prime}\right)^{2} w_{p}\right\|_{2} \leqq C\left\|f_{p}\right\|_{2}$ then (177) yields a contradiction (for sufficiently small $\varepsilon$ ) since $\left\|f_{p}\right\|_{2} \leqq C \varepsilon^{-3 / 2}$. Thus $\left\|f_{p}\right\|_{2} \ll\left\|\left(\nabla^{\prime}\right)^{2} \hat{v}_{p}\right\|_{\infty} \|\left(\nabla^{\prime}\right)^{2}$ $w_{p} \|_{2}$. Taking

$$
\delta=\left\|f_{p}\right\|_{L^{2}}^{1 / 2}\left\|\left(\nabla^{\prime}\right)^{2} \hat{v}_{p}\right\|_{L^{\infty}}^{-1 / 2}\left\|\left(\nabla^{\prime}\right)^{2} w_{p}\right\|_{L^{2}}^{-1 / 2} \ll 1
$$

we obtain

$$
\varepsilon^{-3} \leqq C\left\|f_{p}\right\|_{L^{2}}^{1 / 4}\left\|\left(\nabla^{\prime}\right)^{2} \hat{v}_{p}\right\|_{L^{\infty}}^{3 / 4}\left\|\left(\nabla^{\prime}\right)^{2} w_{p}\right\|_{L^{2}}^{3 / 4},
$$

and comparison with (177) gives the desired lower bounds.

\subsection{Summary}

We have found that for nonzero Poisson's ratio (that is, for $\beta \neq 0$ ) there are two critical loads $0<q_{\text {cr }}<p_{\text {cr }}$ leading to three different regimes.

- For $p \leqq q_{\mathrm{cr}} \mathrm{vK}$ theory is valid and the corresponding minimizer is trivial.

- For $q_{\text {cr }} \leqq p<p_{\text {cr }}$ vK theory is valid and a bifurcation occurs in vK. The minimum of the $\mathrm{vK}$ functional corresponds to a nontrivial out-of-plane displacement (which is not one-dimensional). In terms of the original problem this corresponds to an out-of-plane displacement of order $h$. As $p$ approaches $p_{\text {cr }}$ from below the infimum of the $\mathrm{vK}$ functional goes to $-\infty$ and the norm of the corresponding minimizers explodes. The test functions we have used to show that $\min J_{p}^{\mathrm{vK}} \rightarrow-\infty$ suggest (but do not prove) the following more detailed scenario. For $p$ slightly above $q_{\mathrm{cr}}$ the minimizer involves bending in the $x_{1}$ and in the $x_{2}$ direction of comparable order. In this regime amplitudes are small, the fourth order stretching term is almost negligible and the quadratic form $Q_{2}\left(\left(\nabla^{\prime}\right)^{2} v\right)$ prefers bending in both directions. As $p$ approaches $p_{\text {cr ampli- }}$ tudes become big, the stretching term becomes dominant and we almost have to satisfy the constraint $\operatorname{det}\left(\left(\nabla^{\prime}\right)^{2} v\right)=0$. This favours one-dimensional displacements, and the bending in the $x_{2}$ direction is confined to thinner and thinner boundary layer near $x_{2}=0$ and $x_{2}=l$. The test functions used suggest that the thickness $\delta$ of this boundary layer is of order $p-p_{\mathrm{cr}}$.

- For $p>p_{\mathrm{cr}} \mathrm{vK}$ theory is no longer valid (and the infimum of the $\mathrm{vK}$ functional is $-\infty)$. Instead we are in the Kirchhoff regime where in-plane and out-of-plane displacements are of order 1 . At the critical load the bifurcating eigenfunction corresponds to a one-dimensional deformation.

For zero Poisson's ratio (that is, $\beta=0$ ) we have a different behaviour. For all $p<p_{\mathrm{cr}}$ the $\mathrm{vK}$ theory is valid and the minimizers of $J_{p}^{\mathrm{vK}}$ are trivial (that is, $v=0$ and $u$ satisfies a linear equation and is proportional to $p$ ). Moreover $\min J_{p}^{\mathrm{vK}}$ is uniformly bounded from below. 


\section{The relation between attainment for $J^{\mathrm{vK}}$ and linearized stability}

Here we show that the infimum of $J^{\mathrm{vK}}$ is always attained as long as the linearized stability condition holds, at least for homogeneous boundary data. We consider a slightly more general setting (which includes all the boundary conditions discussed above) since this poses no additional difficulty and highlights the main ideas. We consider an admissible set

$$
\mathcal{A}=\left(u_{0}, v_{0}\right)+X, \quad X=X_{1} \times X_{2},
$$

where

$X_{1}$ is a closed subspace of $W^{1,2}\left(S ; \mathbb{R}^{2}\right)$

$X_{2}$ is a closed subspace of $W^{2,2}(S)$ which contains no nontrivial affine functions.

We also consider the set of linearized isometries in $X$

$$
X^{\text {iso,lin }}:=\left\{(u, v) \in X_{1} \times X_{2}: 2 \operatorname{sym} \nabla^{\prime} u+\nabla^{\prime} v \otimes \nabla^{\prime} v=0\right\}
$$

and the set of infinitesimal in-plane rigid motions

$$
X_{1}^{\text {skew }}:=\left\{u \in X_{1}: u\left(x^{\prime}\right)=W x^{\prime}+c, W^{T}=-W, c \in \mathbb{R}^{2}\right\} .
$$

Suppose that

$$
\left.\begin{array}{l}
F \text { is a bounded linear functional on } W^{2,2}(S), \\
G \text { is a bounded linear functional on } W^{1,2}\left(S ; \mathbb{R}^{2}\right) .
\end{array}\right\}
$$

Typical choices for $F$ and $G$ are the work of the out-of-plane and in-plane forces given by

$$
\begin{aligned}
F(v) & =\int_{S} f v \mathrm{~d} x^{\prime} \\
G(u) & =\int_{\partial S} g \cdot u \mathrm{~d} \mathcal{H}^{1}+\int_{S} h \cdot u \mathrm{~d} x^{\prime} .
\end{aligned}
$$

We study the functional

$$
\begin{aligned}
J_{F, G}^{\mathrm{vK}}:= & \frac{1}{2} \int_{S} Q_{2}\left(\operatorname{sym} \nabla^{\prime} u+\frac{\nabla^{\prime} v \otimes \nabla^{\prime} v}{2}\right) \mathrm{d} x^{\prime} \\
& +\frac{1}{24} \int_{S} Q_{2}\left(\left(\nabla^{\prime}\right)^{2} v\right) \mathrm{d} x^{\prime}-F(v)-G(u) .
\end{aligned}
$$

Definition 26. We say that stability condition (S1) holds if

$$
\begin{aligned}
& J_{0, G}^{\mathrm{vK}}(u, v) \geqq 0 \quad \text { for all }(u, v) \in X^{\text {iso, lin }} \\
& \text { and equality holds only if } v=0 .
\end{aligned}
$$

Note that $(u, v) \in X^{\text {iso,lin }}$ and $v=0$ imply that $u \in X_{1}^{\text {skew }}$. The stability condition (S1) implies that

$$
G(u)=0, \quad \forall u \in X_{1}^{\text {skew }},
$$

since $J_{0, G}^{\mathrm{vK}}(u, 0)=-G(u)$ on the linear space $X_{1}^{\text {skew }}$. 


\subsection{Homogeneous out-of-plane boundary conditions}

We first consider the case that $v=0$ is admissible.

Theorem 27. Assume that $S$ is a bounded Lipschitz domain and that $X, F$ and $G$ satisfy (180) and (183). Suppose further that $u_{0} \in W^{1,2}\left(S ; \mathbb{R}^{2}\right)$ and $v_{0}=0$.

(i) If the stability condition ( $S 1)$ holds for $J_{0, G}^{\mathrm{vK}}$ then $J_{F, G}^{\mathrm{vK}}$ attains its minimum on $\mathcal{A}$ for all $F$.

(ii) If the stability condition (S1) fails then for all $\varepsilon>0$ and all $F$

$$
\inf J_{F,(1+\varepsilon) G}^{\mathrm{vK}}=-\infty \text {. }
$$

Proof. To prove (i) let $\left(u_{0}+u_{j}, v_{j}\right)$ be a minimizing sequence, that is,

$$
J_{F, G}^{\mathrm{vK}}\left(u_{0}+u_{j}, v_{j}\right) \rightarrow \inf _{\mathcal{A}} J_{F, G}^{\mathrm{vK}},
$$

where the infimum may be $-\infty$. Let $\bar{u}_{j} \in X_{1}^{\text {skew }}$ be the best approximation (in $L^{2}$ ) of $u_{j}$ in $X_{1}^{\text {skew }}$ and set

$$
\tilde{u}_{j}=u_{j}-\bar{u}_{j}
$$

Then by (185)

$$
J_{F, G}^{\mathrm{vK}}\left(u_{0}+u_{j}, v_{j}\right)=J_{F, G}^{\mathrm{vK}}\left(u_{0}+\tilde{u}_{j}, v_{j}\right) .
$$

Case $1\left\|\tilde{u}_{j}\right\|_{W^{1,2}}+\left\|v_{j}\right\|_{W^{2,2}}^{2} \leqq C$.

Then a subsequence satisfies $\tilde{u}_{j} \rightarrow \tilde{u}$ in $W^{1,2}, v_{j} \rightarrow v$ in $W^{2,2}$ and hence $v_{j} \rightarrow v$ in $W^{1,4}$ and a standard lower semicontinuity arguments yields $J_{F, G}^{\mathrm{vK}}\left(u_{0}+\tilde{u}, v\right)=$ $\inf _{\mathcal{A}} J_{F, G}^{\mathrm{vK}}$.

Case $2 \lambda_{j}^{2}:=\left\|\tilde{u}_{j}\right\|_{W^{1,2}}+\left\|v_{j}\right\|_{W^{2,2}}^{2} \rightarrow \infty$.

Set

$$
U_{j}=\lambda_{j}^{-2} \tilde{u}_{j}, \quad V_{j}=\lambda_{j}^{-1} v_{j}
$$

Then

$$
\left\|U_{j}\right\|_{W^{1,2}}+\left\|V_{j}\right\|_{W^{2,2}}^{2}=1
$$

and $U_{j}$ is $L^{2}$ perpendicular to $X_{1}^{\text {skew }}$. There exists a subsequence such that

$$
U_{j} \rightarrow U \text { in } W^{1,2}, \quad V_{j} \rightarrow V \quad \text { in } W^{2,2}, \quad V_{j} \rightarrow V \quad \text { in } W^{1,4} .
$$

Now

$$
\begin{aligned}
J_{F, G}^{\mathrm{vK}}\left(u_{0}+\tilde{u}_{j}, v_{j}\right)= & \frac{1}{2} \lambda_{j}^{4} \int_{S} Q_{2}\left(\operatorname{sym} \nabla^{\prime} U_{j}+\frac{\nabla^{\prime} V_{j} \otimes \nabla^{\prime} V_{j}}{2}\right) \mathrm{d} x^{\prime} \\
& +\lambda_{j}^{2} \int_{S} B_{2}\left(\operatorname{sym} \nabla^{\prime} U_{j}+\frac{\nabla^{\prime} V_{j} \otimes \nabla^{\prime} V_{j}}{2}, \operatorname{sym} \nabla^{\prime} u_{0}\right) \mathrm{d} x^{\prime} \\
& +\frac{1}{2} \int_{S} Q_{2}\left(\operatorname{sym} \nabla^{\prime} u_{0}\right)+\frac{1}{24} \lambda_{j}^{2} \int_{S} Q_{2}\left(\left(\nabla^{\prime}\right)^{2} V_{j}\right) \mathrm{d} x^{\prime} \\
& -\lambda_{j}^{2} G\left(U_{j}\right)-G\left(u_{0}\right)-\lambda_{j} F\left(V_{j}\right)
\end{aligned}
$$


where $B_{2}$ is the symmetric bilinear form associated with $Q_{2}$, that is,

$$
4 B_{2}(a, b)=Q_{2}(a+b)-Q_{2}(a-b) .
$$

We claim that, for all $\delta>0$,

$$
\begin{aligned}
& \frac{1}{4} \lambda_{j}^{4} \int_{S} Q_{2}\left(\operatorname{sym} \nabla^{\prime} U_{j}+\frac{\nabla^{\prime} V_{j} \otimes \nabla^{\prime} V_{j}}{2}\right) \mathrm{d} x^{\prime} \\
& \quad+\frac{1}{24}(1-\delta) \lambda_{j}^{2} \int_{S} Q_{2}\left(\left(\nabla^{\prime}\right)^{2} V_{j}\right) \mathrm{d} x^{\prime}-\lambda_{j}^{2} G\left(U_{j}\right) \leqq C_{j} .
\end{aligned}
$$

To see this we first note that, without loss of generality, we may assume that

$$
J_{F, G}^{\mathrm{vK}}\left(u_{0}+\tilde{u}_{j}, v_{j}\right) \leqq J_{F, G}^{\mathrm{vK}}\left(u_{0}, 0\right)+1 \leqq C .
$$

By assumption, $X_{2}$ contains no nontrivial affine functions. This implies that

$$
\|v\|_{W^{2,2}} \leqq C\left\|\left(\nabla^{\prime}\right)^{2} v\right\|_{L^{2}} \quad \forall v \in X_{2}
$$

Hence

$$
\left|\lambda_{j} F\left(V_{j}\right)\right| \leqq \frac{\delta \lambda_{j}^{2}}{24} \int_{S} Q_{2}\left(\left(\nabla^{\prime}\right)^{2} V_{j}\right) \mathrm{d} x^{\prime}+\frac{C}{\delta} .
$$

Since $Q_{2}$ is positive semidefinite the Cauchy-Schwarz inequality yields

$$
\begin{aligned}
& \lambda_{j}^{2}\left|B_{2}\left(\operatorname{sym} \nabla^{\prime} U_{j}+\frac{\nabla^{\prime} V_{j} \otimes \nabla^{\prime} V_{j}}{2}, \operatorname{sym} \nabla^{\prime} u_{0}\right)\right| \\
& \leqq \frac{1}{4} \lambda_{j}^{4} Q_{2}\left(\operatorname{sym} \nabla^{\prime} U_{j}+\frac{\nabla^{\prime} V_{j} \otimes \nabla^{\prime} V_{j}}{2}\right)+Q_{2}\left(\operatorname{sym} \nabla^{\prime} u_{0}\right) .
\end{aligned}
$$

Combining (190), (192), (193) and (188) we arrive at (189). Dividing (189) by $\lambda_{j}^{4}$ we see that

$$
\left\|\operatorname{sym} \nabla^{\prime} U_{j}+\frac{\nabla^{\prime} V_{j} \otimes \nabla^{\prime} V_{j}}{2}\right\|_{L^{2}} \leqq \frac{C}{\lambda_{j}} \rightarrow 0 .
$$

Together with (187) this shows that

$$
\operatorname{sym} \nabla^{\prime} U+\frac{\nabla^{\prime} V \otimes \nabla^{\prime} V}{2}=0,
$$

that is, $(U, V) \in X^{\text {iso,lin }}$.

Dividing (189) by $\lambda_{j}^{2}$ and taking into account the positivity of the first term and weak lower semicontinuity of convex integrals we see that

$$
\frac{1}{24}(1-\delta) \int_{S} Q_{2}\left(\left(\nabla^{\prime}\right)^{2} V\right) \mathrm{d} x^{\prime}-G(U) \leqq 0 .
$$

This holds for all $\delta>0$ and hence also for $\delta=0$, that is,

$$
J_{0, G}^{\mathrm{vK}}(U, V) \leqq 0 \quad \text { and }(U, V) \in X^{\mathrm{iso}, \mathrm{lin}} .
$$


Thus the stability condition (S1) yields $J_{0, G}^{\mathrm{vK}}(U, V)=0$ and $V=0$. This implies that $U \in X_{1}^{\text {skew }}$. By construction $U$ is also $L^{2}$ perpendicular to $X_{1}^{\text {skew }}$. Hence $U=0$. Thus $G\left(U_{j}\right) \rightarrow 0$ and going back to (189) and dividing by $\lambda_{j}^{2}$ we obtain

$$
\limsup _{j \rightarrow \infty} \int_{S} Q_{2}\left(\left(\nabla^{\prime}\right)^{2} V_{j}\right) \mathrm{d} x^{\prime} \leqq 0,
$$

that is, $\left(\nabla^{\prime}\right)^{2} V_{j} \rightarrow 0$ in $L^{2}$ and by (191)

$$
V_{j} \rightarrow 0 \text { in } W^{2,2} \text {. }
$$

Together with (194) this yields sym $\nabla^{\prime} U_{j} \rightarrow 0$ in $L^{2}$. Since $U_{j}$ is perpendicular to $X_{1}^{\text {skew }}$ we can use Korn's inequality to deduce that

$$
U_{j} \rightarrow 0 \text { in } W^{1,2} \text {. }
$$

Combining this with (195) we obtain a contradiction with (186). Thus Case 2 cannot occur and the proof of (i) is finished.

To establish assertion (ii) of the theorem assume now that the stability condition fails. Then there exist $(\bar{u}, \bar{v}) \in X^{\text {iso, lin }}$ such that either $J^{\mathrm{vK}}(\bar{u}, \bar{v})<0$ or $J^{\mathrm{vK}}(\bar{u}, \bar{v})=0$ and $\bar{v} \neq 0$ and thus $\left\|\left(\nabla^{\prime}\right)^{2} \bar{v}\right\|_{L^{2}}>0$. Thus $G(\bar{u})>0$ and $J_{0,(1+\varepsilon) G}^{\mathrm{vK}}(\bar{u}, \bar{v})<0$, for all $\varepsilon>0$. It therefore suffices to show that the assumption $J_{0, G}^{\mathrm{vK}}(\bar{u}, \bar{v})<0$ implies that $\inf _{\mathcal{A}} J_{F, G}^{\mathrm{vK}}=-\infty$.

Since $2 \operatorname{sym} \nabla^{\prime} \bar{u}+\nabla^{\prime} \bar{v} \otimes \nabla^{\prime} \bar{v}=0$ an expansion as in (188) yields

$$
\begin{aligned}
J_{F, G}^{\mathrm{vK}}\left(u_{0}+\lambda^{2} \bar{u}, \lambda \bar{v}\right)= & \frac{1}{2} \int_{S} Q_{2}\left(\operatorname{sym} \nabla^{\prime} u_{0}\right) \mathrm{d} x^{\prime}+\frac{1}{24} \lambda^{2} \int_{S} Q_{2}\left(\left(\nabla^{\prime}\right)^{2} \bar{v}\right) \mathrm{d} x^{\prime} \\
& -\lambda^{2} G(\bar{u})-G\left(u_{0}\right)-\lambda F(\bar{v}) .
\end{aligned}
$$

Thus

$\lim _{\lambda \rightarrow \infty} \frac{1}{\lambda^{2}} J_{F, G}^{\mathrm{vK}}\left(u_{0}+\lambda^{2} \bar{u}, \lambda \bar{v}\right)=\frac{1}{24} \int_{S} Q_{2}\left(\left(\nabla^{\prime}\right)^{2} \bar{v}\right) \mathrm{d} x^{\prime}-G(\bar{u})=J_{0, G}^{\mathrm{vK}}(\bar{u}, \bar{v})<0$.

This finishes the proof of Theorem 27.

\subsection{Non-homogeneous out-of-plane boundary conditions}

We now consider the situation that $v_{0} \notin X_{2}$, that is, $\left(u_{0}, 0\right)$ is not admissible. This corresponds (in the geometrically linear setting of $v \mathrm{~K}$ theory) with choosing a nontrivial reference configuration, for example, a cylindrically bent state rather than a flat state. Thus the behaviour can be very different from that of a flat plate. We first formulate the following weaker stability condition.

Definition 28. We say that $J_{0, G}^{\mathrm{vK}}$ satisfies the stability condition (S2) if

$$
\left.\begin{array}{c}
J_{0, G}^{\mathrm{vK}}(u, v)+\int_{S} \frac{1}{2} Q_{2}\left(\operatorname{sym}\left(\nabla^{\prime} \hat{u}+\nabla^{\prime} v \otimes \nabla^{\prime} \hat{v}\right)\right) \mathrm{d} x^{\prime} \geqq 0 \\
\forall(u, v) \in X^{\text {iso,lin }}, \hat{u} \in X_{1}, \hat{v} \in v_{0}+X_{2} .
\end{array}\right\}
$$


Theorem 29. Suppose that $S$ is a Lipschitz domain, (180) and (183) hold and $u_{0} \in W^{1,2}\left(S ; \mathbb{R}^{2}\right)$ and $v_{0} \in W^{2,2}(S)$.

(i) If the stability condition ( $S 1$ ) holds then $J_{F, G}^{\mathrm{vK}}$ attains its minimum on $\mathcal{A}$, for all $F$.

(ii) If the stability condition (S2) fails then inf $J_{F,(1+\varepsilon) G}^{\mathrm{vK}}=-\infty$, for all $\varepsilon>0$ and all $F$.

Remark 30. From the proof one can extract an abstract stability condition which can be used simultaneously for assertions (i) and (ii). This condition requires that

$$
J_{0, G}^{\mathrm{vK}}(U, V)+\frac{1}{2} Q_{2}\left(\operatorname{sym}\left(M+\nabla^{\prime} v_{0} \otimes \nabla^{\prime} V\right)\right) \mathrm{d} x^{\prime} \geqq 0
$$

for all $(U, V) \in X^{\text {iso,lin }}$ and all $L^{2}$ fields $M$ which arise as weak $L^{2}$ limits of fields $M_{j}$ as in (199) with $\left(U_{j}, V_{j}\right) \rightarrow(U, V)$ and $\lambda_{j} \rightarrow \infty$. We currently do not know how to characterize the class of such fields $M$ more explicitly. Some examples are constructed in the proof of assertion (ii).

Proof. To prove assertion (i) we proceed as in the proof of Theorem 27 and we find $\left(U_{j}, V_{j}\right)$ satisfying (186) and (187), with $U_{j}$ perpendicular to $X_{1}^{\text {skew }}$. The expansion of the energy now yields

$$
\begin{aligned}
J^{\mathrm{vK}} & \left(u_{0}+\lambda_{j}^{2} U_{j}, v_{0}+\lambda_{j} V_{j}\right) \\
= & \frac{1}{2} \lambda_{j}^{4} \int_{S} Q_{2}\left(\operatorname{sym} \nabla^{\prime} U_{j}+\frac{\nabla^{\prime} V_{j} \otimes \nabla^{\prime} V_{j}}{2}\right) \mathrm{d} x^{\prime} \\
& +\lambda_{j}^{3} \int_{S} B_{2}\left(\operatorname{sym} \nabla^{\prime} U_{j}+\frac{\nabla^{\prime} V_{j} \otimes \nabla^{\prime} V_{j}}{2}, \operatorname{sym}\left(\nabla^{\prime} v_{0} \otimes \nabla^{\prime} V_{j}\right) \mathrm{d} x^{\prime}\right. \\
& +\lambda_{j}^{2} \int_{S} B_{2}\left(\operatorname{sym} \nabla^{\prime} U_{j}+\frac{\nabla^{\prime} V_{j} \otimes \nabla^{\prime} V_{j}}{2}, \operatorname{sym} u_{0}+\frac{\nabla^{\prime} v_{0} \otimes \nabla^{\prime} v_{0}}{2}\right) \mathrm{d} x^{\prime} \\
& +\frac{1}{2} \lambda_{j}^{2} \int_{S} Q_{2}\left(\operatorname{sym}\left(\nabla^{\prime} v_{0} \otimes \nabla^{\prime} V_{j}\right)\right) \mathrm{d} x^{\prime} \\
& +\frac{1}{24} \lambda_{j}^{2} \int_{S} Q_{2}\left(\left(\nabla^{\prime}\right)^{2} V_{j}\right) \mathrm{d} x^{\prime}-\lambda_{j}^{2} G\left(U_{j}\right)+\mathcal{O}\left(\lambda_{j}\right)
\end{aligned}
$$

Using the Cauchy-Schwarz inequality for $B_{2}$ as before we can estimate the term involving $\lambda_{j}^{3}$ by a term which can be absorbed into the $\lambda_{j}^{4}$ term and a term of order $\lambda_{j}^{2}$. Thus division by $\lambda_{j}$ yields as before

$$
\left\|\operatorname{sym} \nabla^{\prime} U_{j}+\frac{\nabla^{\prime} V_{j} \otimes \nabla^{\prime} V_{j}}{2}\right\|_{L^{2}} \leqq \frac{C}{\lambda_{j}}
$$

and for a subsequence we have

$$
M_{j}:=\lambda_{j}\left(\operatorname{sym} \nabla^{\prime} U_{j}+\frac{\nabla^{\prime} V_{j} \otimes \nabla^{\prime} V_{j}}{2}\right) \rightarrow M \text { in } L^{2} .
$$


Thus (198) implies that

$$
\begin{aligned}
& \frac{1}{2} \lambda_{j}^{2} \int_{S} Q_{2}\left(M_{j}+\operatorname{sym}\left(\nabla^{\prime} v_{0} \otimes \nabla^{\prime} V_{j}\right)\right) \mathrm{d} x^{\prime} \\
& +\frac{1}{24} \lambda_{j}^{2} \int_{S} Q_{2}\left(\left(\nabla^{\prime}\right)^{2} V_{j}\right)-\lambda_{j}^{2} G\left(U_{j}\right) \leqq \mathcal{O}\left(\lambda_{j}\right) .
\end{aligned}
$$

Therefore

$$
\left.\limsup _{j \rightarrow \infty} \frac{1}{2} \int_{S} Q_{2}\left(M_{j}+\operatorname{sym}\left(\nabla^{\prime} v_{0} \otimes \nabla^{\prime} V_{j}\right)\right) \mathrm{d} x^{\prime}+\frac{1}{24} \int_{S} Q_{2}\left(\nabla^{\prime}\right)^{2} V_{j}\right)-G\left(U_{j}\right) \leqq 0 .
$$

From this we deduce again that $J_{0, G}^{\mathrm{vK}}(U, V) \leqq 0$ and we can finish the argument as in the proof of Theorem 27.

To prove assertion (ii) it again suffices to assume that there exist $(\bar{u}, \bar{v}) \in X^{\text {iso,lin, }}$ $\hat{u} \in X_{1}$ and $\hat{v} \in v_{0}+X_{2}$ such that

$$
J_{0, G}^{\mathrm{vK}}(\bar{u}, \bar{v})+\frac{1}{2} \int_{S} Q_{2}\left(\operatorname{sym}\left(\nabla^{\prime} \hat{u}+\nabla^{\prime} \bar{v} \otimes \hat{v}\right)<0 .\right.
$$

We then need to show $\inf _{\mathcal{A}} J_{F, G}^{\mathrm{vK}}=-\infty$. Consider the test functions

$$
u_{\lambda}=\lambda^{2} \bar{u}+\lambda \hat{u}, \quad v_{\lambda}=\lambda \bar{v}+\hat{v} .
$$

Then

$$
2 \operatorname{sym} \nabla^{\prime} u_{\lambda}+\nabla^{\prime} v_{\lambda} \otimes \nabla^{\prime} v_{\lambda}=2 \lambda \operatorname{sym}\left(\nabla^{\prime} \hat{u}+\nabla^{\prime} \bar{v} \otimes \nabla^{\prime} \hat{v}\right)+\nabla^{\prime} \hat{v} \otimes \nabla^{\prime} \hat{v} .
$$

Thus

$$
\begin{aligned}
\lim _{\lambda \rightarrow \infty} & \frac{1}{\lambda^{2}} J_{F, G}^{\mathrm{vK}}\left(u_{\lambda}, v_{\lambda}\right) \\
\quad & =\frac{1}{2} \int_{S} Q_{2}\left(\operatorname{sym}\left(\nabla^{\prime} \hat{u}+\nabla^{\prime} \bar{v} \otimes \nabla^{\prime} \hat{v}\right) \mathrm{d} x^{\prime}+\frac{1}{24} Q_{2}\left(\left(\nabla^{\prime}\right)^{2} \bar{v}\right) \mathrm{d} x^{\prime}-G(\bar{u})\right. \\
& <0,
\end{aligned}
$$

by (201).

\section{Some facts about isometries and infinitesimal isometries}

\subsection{Construction of isometries}

In this section we recall some general properties of isometries. This is a shortened version of the presentation in $[13,30]$, where detailed proofs can be found. We always deal with maps or functions defined on a bounded Lipschitz domain $S \subset \mathbb{R}^{2}$. To simplify the notation we write $\nabla$ instead of $\nabla^{\prime}$ for the two-dimensional gradient. Given a map $V \in W^{2,2}(S)$ we seek to construct an isometric immersion

$$
y: S \rightarrow \mathbb{R}^{3}, \quad \text { of the form } y=\left(\begin{array}{l}
\Phi \\
V
\end{array}\right) .
$$


We thus need to solve the equation

$$
(\nabla y)^{T} \nabla y=(\nabla \Phi)^{T} \nabla \Phi+\nabla V \otimes \nabla V=I d .
$$

One key result is that (for simply connected domains) the condition $\operatorname{det} \nabla^{2} V=0$ is necessary and sufficient for this, see Theorem 33 below. The same condition is sufficient and necessary to obtain a 'linearized' isometric immersion, that is, a solution of

$$
\nabla W+(\nabla W)^{T}+\nabla V \otimes \nabla V=0
$$

where $W: S \rightarrow \mathbb{R}^{2}$, see Proposition 36 below.

To put these results in perspective we first review some general properties of isometric immersions for the convenience of the reader. These properties are classical for smooth maps, but we will need them for $W^{2,2}$ maps. For a general $W^{2,2}$ map $y: S \rightarrow \mathbb{R}^{3}$ we define the induced metric by $g_{i j}=y_{, i} \cdot y_{, j}$ and we set $n=y_{, 1} \wedge y_{, 2}$ and

$$
A_{i j}=-y_{, i j} \cdot n \text {. }
$$

If $y$ is an isometric immersion, that is, if $g_{i j}=\delta_{i j}$, then $n$ is the normal to the image of $y$ and $A$ is the second fundamental form.

Proposition 31. Suppose that $S$ is a bounded Lipschitz domain and $y \in W^{2,2}\left(S ; \mathbb{R}^{3}\right)$ is an isometric immersion. Then

$$
\begin{gathered}
y_{, i j}=-A_{i j} n, \\
A_{i 1,2}=A_{i 2,1}, \text { for } i=1,2,
\end{gathered}
$$

in the sense of distributions. Moreover

$$
\operatorname{det} A=0 .
$$

Remark 32. If $y$ is smooth then one can deduce from (206) that locally the image of $\nabla y$ is either a constant or a smooth curve. In the latter case one can further conclude that $\nabla y$ is constant on lines defined by the kernel of $A$. It turns out that the latter conclusion is still true for $y \in W^{2,2}$ (see Theorem 41 below). The proof, however, requires a finer analysis $[21,31,34,35]$. The following arguments do not require this geometric property, except for the fine regularity estimates in Section 9.3.

Now we come the announced result on the construction of isometric immersion from linearized isometric immersions.

Theorem 33. [13] Let $S$ be a bounded, simply connected domain with Lipschitz boundary. Suppose that $V \in W^{2,2}(S)$ and $\|\nabla V\|_{L^{\infty}}<1$. Then there exists $\Phi \in W^{1,2}(S)$ with det $\Phi>0$ and

$$
(\nabla \Phi)^{T} \nabla \Phi=I d-\nabla V \otimes \nabla V
$$


if and only of

$$
\operatorname{det} \nabla^{2} V=0 \text {. }
$$

Moreover $\Phi$ is unique up to a rigid motion. If (208) holds and $\|\nabla V\|_{L^{\infty}} \leqq 1 / 2$ then $\Phi$ can be chosen such that $U:=\Phi-i d$ satisfies

$$
\begin{gathered}
\left\|\nabla^{2} U\right\|_{L^{2}} \leqq C\|\nabla V\|\left\|_{L^{\infty}}\right\| \nabla^{2} V \|_{L^{2}}, \\
\|U\|_{W^{2,2}} \leqq C\|\nabla V\| L_{L^{\infty}}\left\|\nabla^{2} V\right\|_{L^{2}}+C\|\nabla V\|_{L^{2}}^{2} .
\end{gathered}
$$

Remark 34. We will see in Proposition 37 that for $V \in W^{2,2}$ the condition (208) actually implies that $V \in C^{1}(S)$. If $S$ is of class $C^{1, \alpha}$ then $\nabla V$ is continuous up to the boundary, see Theorem 39 below.

Remark 35. The precise equations for $\nabla \Phi$ are as follows. Let $a=\nabla V$, $g=I d-a \otimes a$ and let

$$
F=g^{1 / 2}=I d-\lambda a \otimes a, \quad \text { where } \lambda=\frac{1-\sqrt{1-|a|^{2}}}{|a|^{2}} .
$$

Then

$$
\nabla \Phi=\left(\begin{array}{cc}
\cos \theta & -\sin \theta \\
\sin \theta & \cos \theta
\end{array}\right) F
$$

where the derivatives of $\theta$ are given by

$$
\theta_{, j}=\frac{\lambda}{1-\lambda|a|^{2}} a_{, j} \wedge a .
$$

We also recall the classical counterpart of the theorem above for infinitesimal isometries, see, for example, [13].

Proposition 36. Suppose that $S$ is a simply connected, bounded Lipschitz domain. Let $V \in W^{2,2}(S)$. Then the equation

$$
2 \operatorname{sym} \nabla W+\nabla V \otimes \nabla V=0
$$

admits a solution $W \in W^{1,2}\left(S ; \mathbb{R}^{2}\right)$ if and only if

$$
\operatorname{det} \nabla^{2} V=0 .
$$

If (215) holds then $W \in W^{2,2}\left(S ; \mathbb{R}^{2}\right)$ and

$$
W_{i, j k}=-V_{, i} V_{, j k}
$$

In particular det $\nabla^{2} W_{i}=0$, for $i=1,2$. Moreover $W$ is uniquely determined up to an affine map with skew-symmetric gradient. In particular one can choose $W$ such that

$$
\begin{gathered}
\left\|\nabla^{2} W\right\|_{L^{2}} \leqq C\|\nabla V\|\left\|_{L^{\infty}}\right\| \nabla^{2} V \|_{L^{2}}, \\
\|W\|_{W^{2,2}} \leqq C\|\nabla V\|\left\|_{L^{\infty}}\right\| \nabla^{2} V\left\|_{L^{2}}+C\right\| \nabla V \|_{L^{2}}^{2}
\end{gathered}
$$




\subsection{Simple regularity estimates}

In general, functions in $W^{2,2}(S)$ just fail to be in $C^{1}$. The situation is, however, better for isometric immersions. We begin with a scalar result.

Proposition 37. Suppose that $V \in W^{2,2}(S)$ and $\operatorname{det} \nabla^{2} V=0$. Then $V \in C^{1}(S)$. If $B_{\rho}(x) \subset B_{R}(x) \subset S$ we have more precisely

$$
\operatorname{osc}_{B_{\rho}} \nabla V \leqq C\left(\ln \frac{R}{\rho}\right)^{-1 / 2}\left\|\nabla^{2} V\right\|_{L^{2}\left(B_{R}\right)},
$$

where $\operatorname{osc}_{B_{\rho}} f:=\operatorname{diam} f\left(B_{\rho}\right)$.

Proof. Following KIRChHeIM [21] we consider the map $f_{\delta}(x)=\nabla V+\delta\left(-x_{2}, x_{1}\right)$. Then det $\nabla f_{\delta}=\delta^{2}>0$ and the assertion follows from the regularity results of VodyapunOv and Goldstein [40] (see also [10,17,37]) in the limit $\delta \rightarrow 0$.

Now each component of an isometric immersion satisfies det $\nabla^{2} y_{i}=0$ (see Proposition 31). Hence we obtain the following corollary.

Corollary 38. Let $S, V, \Phi$ and $U$ be as in Theorem 33. Then $V, \Phi$ and $U$ are $C^{1}$ in $S$. Moreover, for any compactly contained subset $S^{\prime}$ we have

$$
\|\nabla U\|_{L^{\infty}\left(S^{\prime}\right)} \leqq C\left(S^{\prime}\right)\|\nabla V\|_{L^{\infty}(S)}\left\|\nabla^{2} V\right\|_{L^{2}(S)} .
$$

\subsection{Refined regularity estimates}

We begin with a version of Proposition 37 which holds up to the boundary.

Theorem 39. Suppose that $S$ is a $C^{1, \alpha}$ domain (for some $\alpha>0$ ) and that $V \in W^{2,2}(S)$ with det $\nabla^{2} V=0$. Then $V \in C^{1}(\bar{S})$ and for sufficiently small $\rho, R$ with $0<\rho<R$ we have

$$
\operatorname{osc}_{B_{\rho} \cap S} \nabla V \leqq C\left(\ln \frac{R}{\rho}\right)^{-1 / 2}\left\|\nabla^{2} V\right\|_{L^{2}\left(B_{R} \cap S\right)},
$$

In particular

$$
\|\nabla V\|_{L^{\infty}(S)} \leqq \frac{1}{|S|}\left|\int_{S} \nabla V \mathrm{~d} x\right|+C\left\|\nabla^{2} V\right\|_{L^{2}(S)} .
$$

Remark 40. The result does not hold for Lipschitz domains. Consider for example the truncated cone $\left\{\left(x_{1}, x_{2}\right): x_{1} \in(0,1),\left|x_{2}\right|<x_{1}\right\}$ and $v(x)=v\left(x_{1}\right)$ with $v^{\prime}(0)=\infty$ and $\int_{0}^{1} t\left|v^{\prime \prime}(t)\right|^{2}<\infty$. One may take, for example, $v^{\prime}(t)=|\ln t|^{\alpha}$, $0<\alpha<1 / 2$. A slight modification shows that even $C^{1}$ domains are not sufficient. One needs a certain logarithmic modulus of continuity of the normal.

Proof. See [30]. 
In the setting of Theorem 33 we thus obtain for $C^{1, \alpha}$ domains the estimates

$$
\begin{gathered}
\|\nabla V\|_{L^{\infty}(S)} \leqq C\|V\|_{W^{2,2}(S)} \\
\|\nabla U\|_{L^{\infty}(S)}+\left\|\nabla^{2} U\right\|_{L^{2}(S)} \leqq C\|V\|_{W^{2,2}(S)}^{2}
\end{gathered}
$$

The proof of Theorem 39 uses the fact that the gradient of an isometric immersion is either locally constant or constant along a line segment which touches $\partial S$ at both ends. This is classical for smooth maps. For $C^{2}$ maps this follows from more general results of HARTMAN and NIRENBERG [16]. Under very weak assumptions this characterization was obtained by Pogorelov [34, Chapter II], [35, Chapter IX]. A shorter proof for $W^{2,2}$ maps was recently given by PAKZAD [31], using ideas of KirCHHEIM [21]. We state Pakzad's version for scalar functions.

Theorem 41. Let $S$ be a bounded Lipschitz domain. Suppose that $V \in W^{2,2}(S)$ with det $\nabla^{2} V=0$. Consider the open set

$$
S_{1}=\{x \in S: \nabla V \text { is constant in a neighbourhood of } x\} .
$$

Then through every point $x \in S \backslash S_{1}$ there exists a line segment which intersects $\partial S$ at both ends and on which $\nabla V$ is constant.

The same characterization holds for an isometric immersion in $W^{2,2}\left(S ; \mathbb{R}^{3}\right)$.

Remark 42. Note that $W^{1,2}$ functions on a two-dimensional domain have traces on line segments. Thus constancy along a line segment is a well-defined property. The statement for isometric immersions follows from that for scalar functions as follows. By Proposition 31 the second fundamental form $A$ is curl-free and thus can be locally written as $A=\nabla f$. Since $A$ is symmetric we also have locally $f=\nabla V$. Hence $\operatorname{det} \nabla^{2} V=\operatorname{det} A=0$. Thus if $f$ is not locally constant, it is constant on a line segment. Now (204) and Lemma 45 imply that for each component $y_{i}$ the gradient $\nabla y_{i}$ is constant on that segment.

The above characterization can also be used to approximate $W^{2,2}$ functions with det $\nabla^{2} V=0$ by functions in $W^{2,2} \cap W^{1, \infty}$ satisfying the same constraint even for general Lipschitz domains (cf. Remark 40). The idea is that each component of the set $\{|\nabla V|<r\}$ is bounded by line segments on which $\nabla V$ is constant and by pieces of $\partial S$. If $r$ is sufficiently big then by local regularity there is one large component $U$ of $\{|\nabla V|<r\}$ and we obtain a good approximation by replacing $V$ by a constant in the regions between $\partial U$ and $\partial S$. The precise statement is as follows.

Theorem 43. Suppose that $S$ is a bounded Lipschitz domain. Let $V \in W^{2,2}(S)$ with det $\nabla^{2} V=0$. Then there exists a sequence $r_{k} \rightarrow \infty$, open sets $S_{k} \subset S$ and $V_{k} \in W^{2,2}(S)$ such that

$$
\begin{gathered}
V_{k}=V \quad \text { in } S_{k}, \quad \nabla^{2} V_{k}=0 \quad \text { in } S \backslash S_{k}, \\
\left|\nabla V_{k}\right| \leqq k \quad \text { in } S, \quad\left|\nabla V_{k}\right|=k \quad \text { in } S \backslash S_{k}, \\
\lim _{k \rightarrow \infty}\left|S \backslash S_{k}\right|=0 .
\end{gathered}
$$

In particular $\left\|\nabla^{2} V_{k}\right\|_{L^{2}(S)} \leqq\left\|\nabla^{2} V\right\|_{L^{2}(S)}, V_{k} \rightarrow V$ in $W^{2,2}(S)$ and $\operatorname{det} \nabla^{2} V_{k}=0$. 
Proof. See [30].

Remark 44. If $\Gamma \subset \partial S$ is connected (that is, an interval) and $\nabla V=0$ on $\Gamma$ (in the sense of trace) then we can achieve that $V_{k}=V$ and $\nabla V_{k}=\nabla V=0$ on $\Gamma$. In fact this equality holds in a small open set in $S$ whose boundary contains $\Gamma$.

Above we have used the fact that if $\nabla u$ and $\nabla v$ are parallel in an $L^{2}$ sense in $S$ and if $v$ is constant on a line so is $u$. The following lemma gives a precise statement.

Lemma 45. Let $\Gamma=\left\{\left(x_{1}, x_{2}\right): x_{2}=h\left(x_{1}\right), x_{1} \in(0, a)\right\}$ be a Lipschitz graph and let

$$
U=\left\{\left(x_{1}, x_{2}\right): h\left(x_{1}\right)<x_{2}<h\left(x_{1}\right)+b, x_{1} \in(0, a)\right\}
$$

be a strip above $\Gamma$. Suppose that $u \in W^{1,1}(U)$ and $b_{k}, v_{k} \in W^{1,2}(U)$ and

$$
\nabla u=\sum_{k=1}^{K} b_{k} \nabla v_{k} .
$$

If the functions $v_{k}$ are constant on $\Gamma$ (in the sense of trace) then $u$ is constant on $\Gamma$.

Proof. See [13].

Acknowledgments. This work was carried out while both authors were at the MPI for Mathematics in the Sciences in Leipzig. It is based on a long and very fruitful collaboration with G. Friesecke and R.D. James. We would like to thank in particular G. Friesecke for suggesting to prove the equivalence of linearized and nonlinear instability, based on the corresponding result in one dimension. We would also like to thank L. TRUSKINOVKSY for many interesting discussions and for providing us with a copy of [15] prior to publication. We are also grateful to F. DUDERSTADT for bringing the work of Boobnoff to our attention. ML was partially supported by the RTN HPRN-CT-2002-00282. SM was partially supported by the TMR network FMRX-CT98-0229 and the Marie Curie research training network MRTN-CT-2004-505226. SM wishes to thank Caltech, the University of Minnesota and the IMA, Hebrew University, Beijing University, CISM in Udine and the Mathematical Research Institute Oberwolfach for their hospitality.

\section{References}

1. Acerbi, E., Buttazzo, G., Percivale, D.: A variational definition of the strain energy for an elastic string. J. Elast. 25, 137-148 (1991)

2. Воовnoff, I.G. (И. Г. Бубнов): On a stress of a ships bottom plating due to water pressure. Inst. Naval Arch. Trans. 44, 15-52 (1902)

3. ВоовNOFF, I.G.: Труды по теории пластин (Works on plate theory). Gostechizdat, Moscow, 1953

4. Ciarlet, P.G.: A justification of the von Kármán equations. Arch. Rational Mech. Anal. 73, 349-389 (1980)

5. Ciarlet, P.G.: Mathematical Elasticity II-Theory of Plates. Elsevier, Amsterdam, 1997

6. Duderstadt, F.: Anwendung der von Kármán'schen Plattentheorie und der Hertz'schen Pressung für die Spannungsanalyses zur Biegung von GaAs-Wafern im modifizierten Doppelringtest (Application of the von Kármán plate theory and Hertz' contact for the stress analysis of the bending of GaAs wafers in the modified double-ring test). Dissertation, TU Berlin, 2003 
7. Egorov, A.A.: On stability of classes of affine mappings. Siberian Math. J. 36, 930-942 (1995) ([Sibirskii Mat. Zh. 36, 1081-1095 (1995)]; correction in [8])

8. Egorov, A.A.: Letter to the editors: "On the stability of classes of affine mappings". Siberian Math. J. 42, 1201-1202 (2001) (Sibirskii Mat. Zh. 42, 1443-1444 (2001))

9. FöpPL, A.: Vorlesungen über technische Mechanik, vol. 5. Leipzig, 1907

10. Fonseca, I., Gangbo, W.: Degree theory in analysis and applications. Oxford University Press, London, 1995

11. Friesecke, G., James, R.D., Müller, S.: Rigorous derivation of nonlinear plate theory and geometric rigidity. C. R. Acad. Sci. Paris. Sér. I 334, 173-178 (2002)

12. Friesecke, G., James, R.D., Müller, S.: A theorem on geometric rigidity and the derivation of nonlinear plate theory from three-dimensional elasticity. Comm. Pure Appl. Math. 55, 1461-1506 (2002)

13. Friesecke, G., James, R.D., Müller, S.: A hierarchy of plate models, derived from nonlinear elasticity by Gamma-convergence. Arch. Ration. Mech. Anal. 180, 183-236 (2006)

14. Geymonat, G., Suquet, P.: Functional spaces for Norton-Hoff materials. Math. Meth. Appl. Sci. 8, 206-222 (1986)

15. Grabovsky, Y., Truskinovsky, L.: The flip side of buckling preprint. Cont. Mech. Thermodyn. (2006, to appear)

16. Hartmann, P., Nirenberg, L.: On spherical image maps whose Jacobians do not change sign. Am. J. Math. 81, 901-920 (1959)

17. Iwaniec, T., Šverák, V.: On mappings with integrable dilatation. Proc. Am. Math. Soc. 118, 181-188 (1993)

18. John, F.: Rotation and strain. Comm. Pure Appl. Math. 14, 391-413 (1961)

19. JoHn, F.: Bounds for deformations in terms of average strains. (Ed. O. SHISHA), Inequalities III, pp. 129-144, 1972

20. VON KÁRMÁN, T.: Festigkeitsprobleme im Maschinenbau, in Encyclopädie der Mathematischen Wissenschaften, vol. IV/4, pp. 311-385. Leipzig, 1910

21. Kirchineim, B.: Geometry and rigidity of microstructures. Habilitation thesis, University of Leipzig, 2001. See also: Lecture Notes 16/2003, MPI-MIS, Leipzig http:// www.mis.mpg.de/preprints/ln/lecturenote-1603-abstr.html

22. Kirchinoff, G.: Über das Gleichgewicht und die Bewegung einer elastischen Scheibe. J. Reine Angew. Math. 40, 51-88 (1850)

23. Kopylov, A.P.: Stability of isomteric mappings. Siberian Math. J. 25, 274-283 (1984) (Sibirskii Mat. Zh. 25, 132-144 (1984))

24. LeDret, H., Raoult, A.: Le modéle de membrane non linéaire comme limite variationelle de l'élasticité non linéaire tridimensionelle. C. R. Acad. Sci. Paris Sér. I 317, 221-226 (1993)

25. LeDret, H., Raoult, A.: The nonlinear membrane model as a variational limit of nonlinear three-dimensional elasticity. J. Math. Pures Appl. 73, 549-578 (1995)

26. LeDret, H., Raoult, A.: The membrane shell model in nonlinear elasticity: a variational asymptotic derivation. J. Nonlinear Sci. 6, 59-84 (1996)

27. LiU, F.-C.: A Lusin property of Sobolev functions. Indiana U. Math. J. 26, 645-651 (1977)

28. Monneau, R.: Justification of nonlinear Kirchhoff-Love theory of plates as the application of a new singular inverse method. Arch. Rational Mech. Anal. 169, 1-34 (2003)

29. Morgenstern, D.: Herleitung der Plattentheorie aus der dreidimensionalen Elastizitätstheorie (Derivation of plate theory from three-dimensional elasticity theory). Arch. Ration. Mech. Anal. 4, 145-152 (1959)

30. Müller, S., PAKZAD, R.: Regularity properties of isometric immersions. Math. Z. 251, $1-20(2005)$

31. Pakzad, R.: On the Sobolev space of isometric immersions. J. Differ. Geom. 66, 47-69 (2004)

32. Pantz, O.: Une justification partielle du modèle de plaque en flexion par $\Gamma$-convergence. C. R. Acad. Sci. Paris Sér. I 332, 587-592 (2001) 
33. Pantz, O.: On the justification of the nonlinear inextensional plate model. Arch. Rational Mech. Anal. 167, 179-209 (2003)

34. Pogorelov, A.V.: Surfaces with Bounded Extrinsic Curvature (Russian). Kharhov, (1956)

35. Pogorelov, A.V.: Extrinsic Geometry of Convex Surfaces, Translation of mathematical monographs. vol. 35. American Math. Soc., 1973

36. ReshetNYAK, Y.G.: Liouville's theory on conformal mappings under minimial regularity assumptions. Siberian Math. J. 8, 69-85 (1967) (Sibirskii Mat. Zh. 8, 91-114 (1967))

37. Š́Verák, V.: Regulartiy properties of deformations with finite energy. Arch. Rational Mech. Anal. 100, 105-127 (1988)

38. Truesdell, C.: Comments on rational continuum mechanics. Three lectures for the International Symposium on Continuum Mechanics and Partial Differential Equations, Instituto de Matemática, University Federal do Rio de Janeiro, August 1-5, 1977. Contemporary developments in continuum mechanics and partial differential equations (Eds. G.M. LaPenha, L.E. Medeiros), pp. 495-603. North-Holland, Amsterdam, 1978

39. Villaggio, P.: Mathematical Models for Elastic Structures. Cambrigde University Press, London, 1997

40. Vodopyanov, S.K., Goldstein, V.M.: Quasiconformal mappings and spaces with generalized first derivatives. Siberian Math. J. 17, 399-411 (1976) (Sibirskii Mat. Zh. 17, 515-531 (1976))

41. Ziemer, W.: Weakly Differentiable Functions. Springer, New York, 1989

Max Planck Institute for Mathematics in the Sciences,

Inselstr. 22-26,

04103 Leipzig, Germany.

e-mail: Myriam.Lecumberry@ mis.mpg.de

and

Hausdorff Center for Mathematics

\& Institute for Applied Mathematics,

Bonn University,

Endenicher Allee 60,

53115 Bonn, Germany.

e-mail: sm@hcm.uni-bonn.de

(Received November 12, 2006 / Accepted February 22, 2007)

Published online May 5, 2009 - (C) Springer-Verlag (2009) 